\title{
(Universal) Unconditional Verifiability in E-Voting without Trusted Parties
}

\author{
Gina Gallegos-Garcia ${ }^{1}$, Vincenzo Iovino ${ }^{2}$, Alfredo Rial ${ }^{2}$, Peter B. Rønne ${ }^{2}$, and \\ Peter Y. A. Ryan ${ }^{2}$ \\ 1 Instituto Politecnico Nacional, Mexico \\ ggallegosg@ipn.mx \\ 2 University of Luxembourg \\ vinciovino@gmail.com, \{alfredo.rial@uni.lu, peter.roenne, \\ peter.ryan\}@uni.lu
}

\begin{abstract}
In e-voting protocol design, cryptographers must balance usability and strong security guarantees, such as privacy and verifiability. In traditional e-voting protocols, privacy is often provided by a trusted authority that learns the votes and computes the tally. Some protocols replace the trusted authority by a set of authorities, and privacy is guaranteed if less than a threshold number of authorities are corrupt. For verifiability, stronger security guarantees are demanded. Typically, corrupt authorities that try to fake the result of the tally must always be detected.

To provide verifiability, many e-voting protocols use Non-Interactive Zero-Knowledge proofs (NIZKs). Thanks to their non-interactive nature, NIZKs allow anybody, including third parties that do not participate in the protocol, to verify the correctness of the tally. Therefore, NIZKs can be used to obtain universal verifiability. Additionally, NIZKs also improve usability because they allow voters to cast a vote using a non-interactive protocol.

The disadvantage of NIZKs is that their security is based on setup assumptions such as the common reference string (CRS) or the random oracle (RO) model. The former requires a trusted party for the generation of a common reference string. The latter, though a popular methodology for designing secure protocols, has been shown to be unsound.

In this paper, we address the design of an e-voting protocol that provides verifiability without any trust assumptions, where verifiability here is meant without eligibility verification. We show that Non-Interactive Witness-Indistinguishable proofs (NIWI) can be used for this purpose. The e-voting scheme is private under the Decision Linear assumption, while verifiability holds unconditionally. To our knowledge, this is the first private e-voting scheme with perfect universal verifiability, i.e. one in which the probability of a fake tally not being detected is 0 , and with non-interactive protocols that does not rely on trust assumptions.
\end{abstract}

Keywords: e-voting, verifiability, witness indistinguishability, bilinear maps. 


\section{Table of Contents}

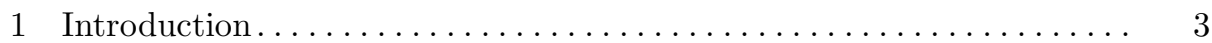

1.1 Background and Statement of the Problem .............. 3

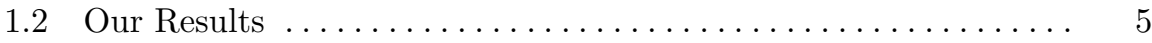

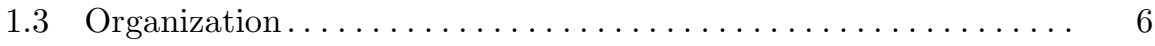

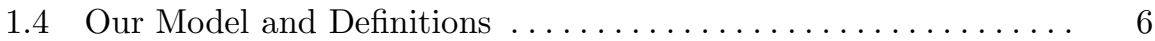

Privacy .....................................

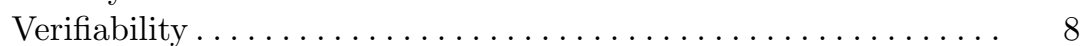

On the Need of a Stronger Correctness Property ............. 9

1.5 Warm-up: Our Weakly Verifiable eVote (Sketch) ........... 9

Intuition ................................ 10

Sketch of the construction ..................... 10

Weak Verifiability of the Construction $\ldots \ldots \ldots \ldots \ldots \ldots \ldots . \ldots \ldots$

Weak Privacy of the Construction ................... 12

1.6 Our Fully Verifiable eVote (Sketch) . . . . . . . . . . . . . . 13

Sketch of the Construction ......................... 14

(Full) Verifiability of the Construction ............... 15

(Full) Privacy of the Construction ................. 16

1.7 eVote with Multiple Authorities and Threshold Privacy ....... 18

Sketch of the Construction ......................... 18

Verifiability of the Construction ..................... 19

Privacy of the Construction.......................... 20

1.8 On The Reusability of the Public Parameters ............ 21

1.9 Related Work .................................... 22

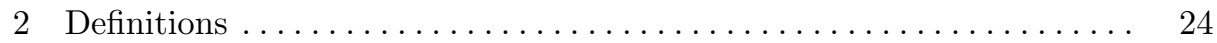

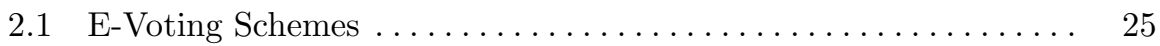

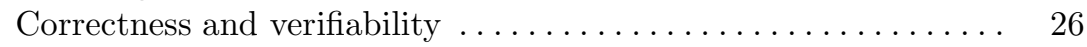

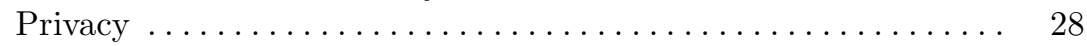

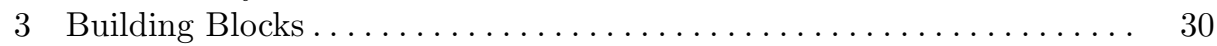

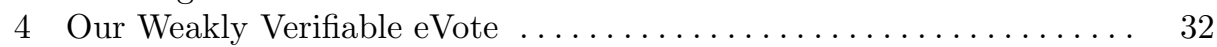

4.1 Correctness and Weak Verifiability of the Construction ......... 33

4.2 Weak Privacy of the Construction $\ldots \ldots \ldots \ldots \ldots \ldots \ldots \ldots$

5 Our (Fully) Verifiable eVote ........................... 39

5.1 Correctness and (Full) Verifiability of the Construction........ 42

5.2 Privacy of the Construction ....................... 45

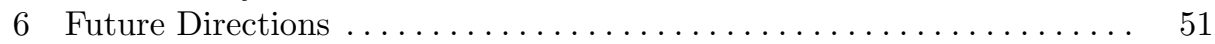

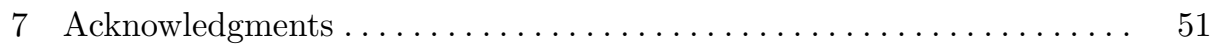




\section{Introduction}

\subsection{Background and Statement of the Problem}

The parties participating in a standard e-voting protocol are multiple voters and one authority. First, the authority sets up a public key retaining a corresponding secret key. A voter computes a ballot on input the public key of the authority and her intended vote and sends the ballot to a write-only public bulletin board (PBB), which records it in an entry associated with that voter. In case of abstention, a special symbol $\perp$ is recorded on the PBB. The authority uses its secret key to compute the tally on input all the ballots on the PBB, which could possibly be $\perp$ in case of abstention. Finally, the correctness of the tally can be checked by running a verification algorithm 1

E-voting protocols must provide two security properties: privacy and verifiability. Privacy should protect the secrecy of the votes. Verifiability should prevent a corrupt authority from faking the tally. We will provide a formal definition of verifiability that is stronger than previous ones in some respects.

Privacy protection assumes the existence of a trusted authority in many evoting systems [Cha81|CGS97|DJ01|RS06|Adi08 $\mathrm{CCC}^{+}$09 RT09|JCJ10]. As for schemes that distribute the trust among several authorities, privacy protection still requires that not all of the authorities are corrupt. Nevertheless, verifiability (also called integrity) should be guaranteed even if the authorities are corrupt.

Many e-voting systems make use of Non-Interactive Zero-Knowledge Proofs (NIZK) BFM88DMP88RS92Gol01 $\mathrm{DDO}^{+} 01$ to provide verifiability. NIZK must provide two properties: soundness and zero-knowledge. Soundness prevents a corrupt prover from proving a false statement, i.e., a statement for which no witness exists. Zero-knowledge ensures that the verifier does not learn any information about the witness.

Zero-knowledge is defined following the simulation paradigm, i.e., it requires the existence of a simulator that computes a valid proof without knowledge of the witness. However, if such a simulator existed, soundness would not hold. This apparent contradiction is solved by resorting to trust assumptions like the Common Reference String (CRS) model BFM88. In the CRS model, a trusted party generates a CRS that is used by both provers and verifiers. The simulator is given the additional power of computing the CRS. Thanks to that, the simulator knows trapdoor information that allows it to simulate proofs for all statements.

For some applications of NIZK, the CRS model is not problematic. For instance, in IND-CCA public key encryption schemes [NY90 $\mathrm{DDO}^{+} 01 \mathrm{CS03b}$, zero-knowledge does not need to hold for the receiver of ciphertexts because the receiver must be able to decrypt anyway. Therefore, the CRS is computed by the receiver, while the NIZK proofs are computed by the sender of ciphertexts. However, in e-voting, the authority cannot compute the CRS because it must compute proofs that show the correctness of the tally.

\footnotetext{
${ }^{1}$ In this description we skipped some details (e.g., eligibility and authentication) that are not relevant to our setting. See below for more discussion.
} 
An alternative to the CRS model is the Random Oracle (RO) model BR93. The RO model assumes the availability of a perfect random function available to all parties. NIZKs that use the RO model are constructed following the FiatShamir heuristic [FS87. To prove that a NIZK proof constructed following this heuristic is zero-knowledge, we need programmability of the RO, i.e., the ability of the simulator to change the input/output of the RO.

To compute a proof, in practice, the prover replaces the RO by some "secure" hash function. Therefore, this hash function must be chosen honestly for zeroknowledge to hold. Consequently, all the parties must trust the implementation of a concrete hash function (e.g., SHA-3 [BDPA11]). We note that a hash function could have been designed in a malicious way (e.g., "programmed" like in the simulation) to allow the computation of a proof for a false statement. Currently, this needed trust on the implementation of hash functions does not exist. In fact, different political entities have developed their own hash functions because they do not trust the hash functions designed by others. For instance, the Russian government discourages the use of SHA-3 and encourages the use of its own hash function [Fed12.

Moreover, even when programmability is not needed, the RO methodology has been shown to be unsound [CGH98. Further problems are known regarding the programmability of the RO in the context of NIZK [GK03|Kal06 $\mathrm{BDSG}^{+} 13$. The current techniques to avoid the need of programmability resort to the CRS model [DFN06|Lin15|CG15|CPSV16].

This motivates our main question: is it possible to design an e-voting scheme that is verifiable without assuming any trust assumption (like CRS and RO)?

In a survey Lip05, Lipmaa asks whether Non-Interactive Witness Indistinguishable Proofs (NIWI) can be used to replace NIZKs. NIWIs can be constructed without using any trust assumptions GOS06 DN00|BOV03|BP15]2

NIWI is a non-interactive proof/argument system that provides weaker security guarantees in comparison to NIZKs. While NIZKs ensure that a proof does not reveal any information about the witness, NIWIs only guarantee that, for any two witnesses $w_{1}$ and $w_{2}$ for the same statement, a proof computed with $w_{1}$ is computationally indistinguishable from a proof computed with $w_{2}$. Note that this notion only makes sense for languages with multiple witnesses for each statement, which is not always the case.

To our knowledge, it was not known how to use NIWI to construct an evoting scheme (eVote, in short) that is both private and verifiable. Usually, it is very difficult to use NIWI because of its weaker security guarantee. Nonetheless, inspired by a recent result on functional encryption $\left[\mathrm{BSW11} \mathrm{GGH}^{+} 13\right.$ of Badrinarayanan, Goyal, Jain and Sahai BGJS16, we are surprisingly able to profitably use NIWI to answer our main question affirmatively.

\footnotetext{
${ }^{2}$ Note that, in the literature, there are both NIWIs in the CRS model, like the ones of Groth and Sahai GS08, and one-message NIWIs without CRS (see the citations above). Henceforth, unless specified otherwise, we denote by NIWI the (one-message) variant without CRS, and in particular we refer to the NIWIs for CircuitSat of Groth et al. GOS06.
} 


\subsection{Our Results}

First, we define correctness, privacy and verifiability properties for an eVote. We define two flavors of privacy and verifiability: weak and full. We propose an eVote that is (fully) private and (fully) verifiable. Its privacy can be reduced to the Decision Linear assumption BBS04. Its verifiability is perfect (see below) and thus is not based on any assumption. Moreover, its verifiability is universal, i.e., even a third party who did not participate in the election process should be able to verify the correctness of the tally. As a warm-up, we also describe an eVote that fulfills the weak privacy and weak verifiability properties.

Our eVote uses as building blocks a NIWI proof system, a public key encryption scheme with perfect correctness and unique secret key, and a perfectly binding commitment scheme. It can be instantiated by using just bilinear groups BF03 Jou04. For instance, we can instantiate our construction with the NIWI of Groth, Ostrovsky and Sahai GOS06] and the Decision Linear encryption scheme of Boneh et al. BBS04. When instantiated with those building blocks, our construction is the first eVote with non-interactive algorithms for casting and verifying ballots and for computing and verifying the tally that provides perfect (weak and full) verifiability (as defined in Def. 3) and that fulfills the (weak and full) privacy property under the Decision Linear assumption [BBS04. The Decision Linear assumption is a well-studied assumption over bilinear groups. Our construction attains universal verifiability, i.e., even third parties who did not participate in the election process are able to verify the tally.

We prove that our weakly verifiable eVote fulfills the weak verifiability and weak privacy properties in Corollary 3 , and we prove that our (fully) verifiable eVote fulfills the (full) verifiability and (full) privacy properties in Corollary 6 . We remark that the computational assumption is only needed to prove that our eVotes fulfill the (weak or full) privacy properties. In contrast, no assumption at all is necessary to prove that they fulfill the (weak or full) verifiability properties.

Therefore, our eVote with non-interactive algorithms is the first eVote whose perfect verifiability is not based on any trust and that is provably secure under a well-studied and falsifiable assumption [Nao03. The latter is a key point of our results because otherwise one could just claim that an eVote in the RO model is secure when instantiated with any hash function. However, even when using such "unfalsifiable" assumptions, perfect verifiability cannot be achieved against unbounded adversaries because, in practice, for any hash function whose domain is larger than the range, the probability of finding a collision is not 0 .

In Section 1.7, we outline how to adapt our (fully) verifiable construction to a model with multiple authorities. In this model, the tally evaluation algorithm is run by a set of authorities and the privacy property must hold if at least one authority is honest. (As this is not the main focus of our work, we do not present formal definitions and details for its construction.) An important advantage of our construction is that no interaction among the authorities is required. In this respect, our techniques completely diverge from previous approaches to the problem and may be of independent interest. We stress that the multi-string 
model of Groth and Ostrovsky [GO14], though conceptually appealing in this scenario, fails to provide a solution.

In this work, we use cryptographic primitives to demonstrate the achievability of perfect verifiable systems. However, we are not concerned about usability and "human-friendly" verifiability, as dealt with in Riv06 RR06 RRI16. Furthermore, we only consider traditional e-voting systems and hence we neglect other approaches [KY02|DJ03 Gro04|HRZ10|KSRH12]GIR16].

Our privacy definition is inspired by the one of Benaloh [Ben87, also called "PRIV" in $\mathrm{BCG}^{+} 15$, which we reformulate by using modern terminology and we modify conveniently to withstand the attacks shown in $\mathrm{BCG}^{+} 15$. We believe that our (fully) verifiable eVote can be proven secure according to other definitions of security, like for instance the one of Chase et al. [CKLM13, but we did not investigate the details because it is out of the scope of this initial work.

\subsection{Organization}

We describe the concept of eVote and its verifiability and privacy properties in Section 1.4. In Section 2, we present detailed definitions of an eVote and of its verifiability and privacy properties. In Section 3 we present the building blocks we will use in our constructions.

In Section 1.5 (resp. Section 1.6) we include all major details needed to understand our construction for a weakly verifiable eVote (resp. (fully) verifiable eVote) and its security properties. In Section 4 (resp. Section 5) we present the full details of our construction for a weakly verifiable eVote (resp. fully verifiable eVote) and of its security properties.

In Section 1.7, we outline how to adapt our (fully) verifiable eVote to a model with multiple authorities and threshold privacy. In this model, the tally evaluation algorithm is run by a set of authorities and privacy must hold if at least one of the authorities is honest.

In Section 1.8 we make some additional remarks about our definitions and in particular about the possibility of re-using the parameters through different elections. In Section 1.9 we discuss relevant related works. Finally, in Section 6 we discuss some future directions in cryptography and e-voting that our work opens up.

\subsection{Our Model and Definitions}

In this section, we introduce our definitions of privacy and verifiability. We use a simple e-voting model with a single authority. We remark that, even for this model, it was not known how to avoid the use of CRSs or ROs. In Section 1.7, we outline how to adapt our constructions to a model with multiple authorities. Formal definitions of an eVote and of its privacy and verifiability are given in Section 2.1

We use a general tally function $F:(\mathcal{M} \cup\{\perp\})^{N} \rightarrow\{0,1\}^{\star} \cup\{\perp\}$, where $\mathcal{M}$ is the message space. The special symbol $\perp$ denotes either an invalid vote or a 
blank ballot, when it is input to the function, or an error, when it is output by the function. A voter casts $\perp$ to denote a blank ballot, i.e., a valid ballot where no candidate is chosen. A voter abstains from voting by not casting any ballot or by casting an invalid ballot, which is replaced by $\perp$ in the evaluation phase.

Our general tally function $F$ must satisfy a very natural property given in Def. 2. The messages belong to a message space $\mathcal{M}$ that is not specified. As byproduct, our constructions can be instantiated, for instance, to the case of a YES/NO election with the sum as tally function. As shown in $\mathrm{BCG}^{+} 15$, care has to be taken when considering general tally functions.

Privacy Our privacy definition is indistinguishability-based and states that no PPT adversary can win the following game with non-negligible advantage. The adversary receives the public key generated by a challenger and chooses two tuples of strings that encode either valid votes in the message space $\mathcal{M} \cup\{\perp\}$ or arbitrary ballots, which are cast by possibly corrupt voters. We require that the tally function outputs the same result on input any of the tuples of strings.

The challenger chooses at random one of the two tuples. The challenger runs the ballot verification algorithm on input each of the arbitrary ballots and replaces the arbitrary ballot in the tuple by $\perp$ if verification is unsuccessful. The challenger runs the cast algorithm on input each of the valid votes in the message space to compute a ballot and replaces the valid vote in the tuple by the ballot. Then the challenger computes the tally and a proof of correctness of the tally.

The new tuple, which replaces valid votes by ballots and invalid arbitrary votes by $\perp$, is given to the adversary along with a proof of the correctness of the tally. The adversary guesses which of the two tuples was chosen by the challenger.

More formally, the adversary sends two tuples $V_{0}=\left(m_{0,1}, \ldots, m_{0, N}\right)$ and $V_{1}=\left(m_{1,1}, \ldots, m_{1, N}\right)$ and a set $S \subset[N]$. The set $S$ contains the indices of the strings of arbitrary ballots. For each $j \in S, m_{0, j}=m_{1, j}$ must hold. For each $j \notin S, m_{0, j}, m_{1, j} \in \mathcal{M} \cup\{\perp\}$ must hold. Moreover, we require that for all $d_{1}, \ldots, d_{N} \in \mathcal{M} \cup\{\perp\}, F\left(m_{0,1}^{\prime}, \ldots, m_{0, N}^{\prime}\right)=F\left(m_{1,1}^{\prime}, \ldots, m_{1, N}^{\prime}\right)$ must hold, where, for each $j \in S, m_{0, i}^{\prime}=m_{1, i}^{\prime}=d_{i}$ must hold, and for each $j \notin S, b \in$ $\{0,1\}, m_{b, j}^{\prime}=m_{b, j}$ must hold.

Our definition can be viewed as a variant of Benaloh's ballot privacy definition [Ben87] (also called "PRIV" in $\left.\mathrm{BCG}^{+} 15\right]$ ) reformulated by using modern terminology and corrected to rule out some known attacks [BCG $\left.{ }^{+} 15\right]$.

We also define weak privacy. The difference between the definitions of weak privacy and privacy is that, in weak privacy, the set $S$ must be empty, i.e., the adversary cannot submit arbitrary ballots.

The privacy definitions that we use here are simple and do not capture vote replay attacks, see e.g. [CS10]. Such attacks are easily prevented by enforcing ballot independence. This can e.g. be done by appending a proof of knowledge of the plaintext in the ballots of the voters. Presently, this has not been done in the NIWI setting, so we will disregard this point for clarity. However, we stress that it is easy to change the schemes to satisfy full privacy definitions within the framework of having trust for privacy, but not for verifiability. 
Verifiability We define a ballot verification and a tally verification algorithm. In our definition of verifiability, we require two conditions to hold. The first condition states that, if each ballot and the proof of correctness of the tally issued by the authority are verified successfully by the respective algorithms, then each ballot $C_{i}$ (possibly computed on input a maliciously generated public key) must be associated with a unique message $m_{i} \in \mathcal{M} \cup\{\perp\}$, and the result $y$ claimed by the authority equals $F\left(m_{1}, \ldots, m_{n}\right)$.

The second one requires that, even when the adversary generates the public key, if honest voters cast a ballot that is accepted by the ballot verification algorithm, then the ballot has to be "counted". More concretely, consider that some ballots are computed by honest voters and are accepted by the ballot verification ballot algorithm. (These ballots could be ill-formed if they are computed on input a public key generated by the adversary.) Consider also that the remaining ballots are computed by corrupt voters. In this situation, the tally evaluation algorithm outputs a tally $y$ and a proof of correctness that, along with the public key and the ballots, is accepted by the tally verification algorithm. Then, it must be the case that the ballots sent by honest voters were counted to obtain $y$. For example, if the tally function is a sum function that sums binary votes and three honest voters cast three 1's, then the authority should not be able to claim that $y<3$.

Remarkably, our construction provides perfect verifiability. Perfect verifiability means that the probability that a malicious authority computes an incorrect tally and a proof that are accepted by the tally verification algorithm is null.

We also define weak verifiability. In weak verifiability, the authority can incorrectly claim that $y=\perp$. The second condition described above is still guaranteed for all the tallies $y \neq \perp$. For a weakly verifiable eVote, we only require weak privacy.

Although our weakly verifiable eVote satisfies weaker properties, it represents a worthwhile warm-up. Our (fully) verifiable eVote is based on it, though with some relevant modifications. Our weakly verifiable construction does not need a ballot verification algorithm, but for simplicity we use the same syntax for both the weakly verifiable and (fully) verifiable schemes. In the next subsection, we describe a definition of correctness that is stronger than previous ones. This definition is needed to exclude the case that an eVote that is intuitively not verifiable fulfills formally the definition of verifiability.

In Section 1.7. we outline how to adapt our (fully) verifiable construction to a model with multiple authorities. In this model, the tally is computed by a set of authorities. Privacy must hold if at least one authority is honest. Because this model is not the main focus of our work, we do not present formal definitions or a detailed description of its construction. We note that such a construction would satisfy a different, but still without trust assumptions, definition of verifiability that essentially states that if there is at least one honest voter, the verifiability holds with overwhelming probability over the random coins of such voter, a very minimal assumption. 
In this paper, for simplicity, we do not directly address issues of eligibility. We assume that a ballot is associated with a voter uniquely and that the adversary cannot submit a ballot on behalf of some voters. Unconditional eligibility verifiability seems hard or impossible to achieve, since we normally use some commitment, e.g. a PKI, and digital signatures on the ballots to prove eligibility, but such an approach is not secure against a computationally unbounded adversary.

Our construction can however easily be extended to take into account such attacks by using digital signatures in a standard, but non-perfect, way (see e.g. CGGI14). The resulting construction would nonetheless satisfy a meaningful notion of verifiability secure against computationally bounded adversaries not based on any trust assumption, which advances the state of the art. In fact, to our knowledge, it is not even known how to construct an eVote protocol with computational verifiability without trusted parties.

On the Need of a Stronger Correctness Property We justify here why a stronger correctness property is needed. Traditionally, the correctness property guarantees both (1) that the ballot verification algorithm accepts the ballots computed by the cast algorithm, and (2) that the tally verification algorithm accepts the tally and the proof computed by the tally evaluation algorithm. In the latter, the ballots taken as input by the tally evaluation algorithm are computed by the cast algorithm. Therefore, it is not guaranteed that the tally verification algorithm accepts the output of the tally evaluation algorithm when the ballots are not computed by the cast algorithm.

We explain now that this is an issue. In our weakly verifiable scheme, the ballot verification algorithm accepts any ballot. Therefore, it would be possible to say that such a scheme is (fully) verifiable by just changing the tally verification algorithm so that it accept $y \triangleq \perp$ only when all the ballots equal $\perp$. As can be seen, condition (1) in the definition of (full) verifiability (cf. Def. 3) is fulfilled because the "if part" of the condition never holds.

However, intuitively, such a scheme is incorrect. Namely, if an honest authority that runs tally evaluation algorithm and gets $y=\perp$ (because some ballots where ill-formed), the tally verification algorithm should accept that result.

To address this issue, we add condition (2) to the definition of correctness (cf. Def. 3). This condition states that the tally verification algorithm must accept the output of the tally evaluation algorithm when run on input ballots that are accepted by the ballot verification algorithm (as opposed to ballots computed by the cast algorithm). We point out that in some works on definitional foundations (e.g., Bernhard et al. $\left[\mathrm{BCG}^{+} 15\right]$ ) this issue has been overlooked.

\subsection{Warm-up: Our Weakly Verifiable eVote (Sketch)}

In this section, we sketch our construction for a weakly verifiable eVote, i.e. an eVote that fulfills the weak verifiability and weak privacy properties. A (fully) verifiable eVote, which satisfies (full) verifiability and (full) privacy, is presented 
in Section 1.6. We stress that in practice such weakly verifiable eVote lacks fundamental security guarantees, but nonetheless it serves as a worthwhile warmup to our (fully) verifiable eVote.

Intuition Our weakly verifiable eVote uses 3 instances of a public key encryption (PKE) scheme in parallel. We require that the PKE scheme fulfills two properties: perfect correctness and unique secret key (see Def. 6). PKE schemes with those properties are known in the literature [DH76|BBS04 and can be constructed, e.g., from the Decision Linear assumption [BBS04]. The voter encrypts her vote 3 times using the PKE scheme without adding any proof of ciphertext well-formedness. Therefore, a ballot consists of three ciphertexts.

To compute the tally, the authority proceeds as follows. The authority decrypts the first ciphertext and the second ciphertext in a ballot. The authority replaces decrypted messages that do not belong to the message space by $\perp$.

The authority evaluates the tally function twice. First, the authority uses as input the messages encrypted in the first ciphertext of each ballot. Second, it uses the messages encrypted in the second ciphertext of each ballot. If both tallies are equal, the authority outputs the tally along with a proof of correctness, else the authority returns $\perp$ to indicate an error.

The property of unique secret key guarantees that the decrypted message will be unique for each ciphertext. Without this property, it could be possible that a voter cast an invalid ciphertext $\mathrm{Ct}$ not belonging to the ciphertext space such that the decrypted message is different when using two well-formed secret keys $\mathbf{S k}_{1}$ and $\mathbf{S k}_{2}$ for the same public key. Note that this is not prevented by the correctness property, which only holds when the ciphertext is an output of the encryption algorithm.

Sketch of the construction Let $N$ be the number of voters and let $F$ be a tally function with message space $\mathcal{M}$. The public key $\mathrm{Pk}$ of our eVote consists of the $3 \mathrm{PKs}\left(\mathrm{Pk}_{1}, \ldots, \mathrm{Pk}_{3}\right)$ of the underlying PKE. The secret key consists of the 3 corresponding SKs $\left(\mathrm{Sk}_{1}, \ldots, \mathrm{Sk}_{3}\right)$ of the PKE.

Our cast algorithm takes as input the public key $\left(\mathrm{Pk}_{1}, \ldots, \mathrm{Pk}_{3}\right)$, the index $j$ of the voter 3 (for $j \in[N]$ ), and a vote $v$. The cast algorithm outputs a ballot for the $j$-th voter. Our cast algorithm just encrypts the vote $v$ with the 3 instances of the PKE to produce the ciphertexts $\mathrm{Ct}_{1}, \ldots, \mathrm{Ct}_{3}$. The ballot given as output is $\mathrm{Blt} \triangleq\left(\mathrm{Ct}_{1}, \ldots, \mathrm{Ct}_{3}\right)$.

The tally evaluation algorithm works as follows. For all $j \in[N]$, if the corresponding voter cast her vote, for all $l \in[2]$, decrypt $\mathrm{Ct}_{j, l}$ with $\mathrm{Sk}_{l}$ to get $m_{j, l}$. Then, for all $l \in[2]$ compute $y_{l}=F\left(m_{1, l}, \ldots, m_{N, l}\right)$, where for indices $j$ such

\footnotetext{
${ }^{3}$ The index is needed to associate a ballot with a unique voter. For instance, an eVote could require that each voter encrypts her ballot with a different PKE public key, adding a proof of well-formedness. The public key of the eVote would contain $N$ PKE's public keys, one for each voter, and so the statement of the proof would have to contain the index of the voter in the set $N$.
} 
that either $m_{j, l} \notin \mathcal{M}$ or the $j$-th voter did not cast her vote, we set $m_{j, l}=\perp$. If the two $y_{l}$ 's are equal to the same string $y$ then return this as the tally, otherwise return an error $y=\perp$. Finally, compute a NIWI proof $\gamma$ of the fact that $x=\left(\mathrm{Blt}_{1}, \ldots, \mathrm{Blt}_{N}, \mathrm{Pk}_{1}, \ldots, \mathrm{Pk}_{3}\right)$ satisfies the relation $\mathrm{R}^{\mathrm{dec}}$ in Fig. 1] using as witness $\left(\mathrm{Sk}_{1}, \mathrm{Sk}_{2}, s_{1}, s_{2}\right)$. Another part of the witness is the two indices $i_{1}, i_{2} \in[3]$, $i_{1}<i_{2}$, which determine the two columns of ciphertexts that are used to compute the tally. In the real mode described above, we have $i_{1}=1, i_{2}=2$, but we can also have trapdoor modes with other index choices which will be essential for privacy.

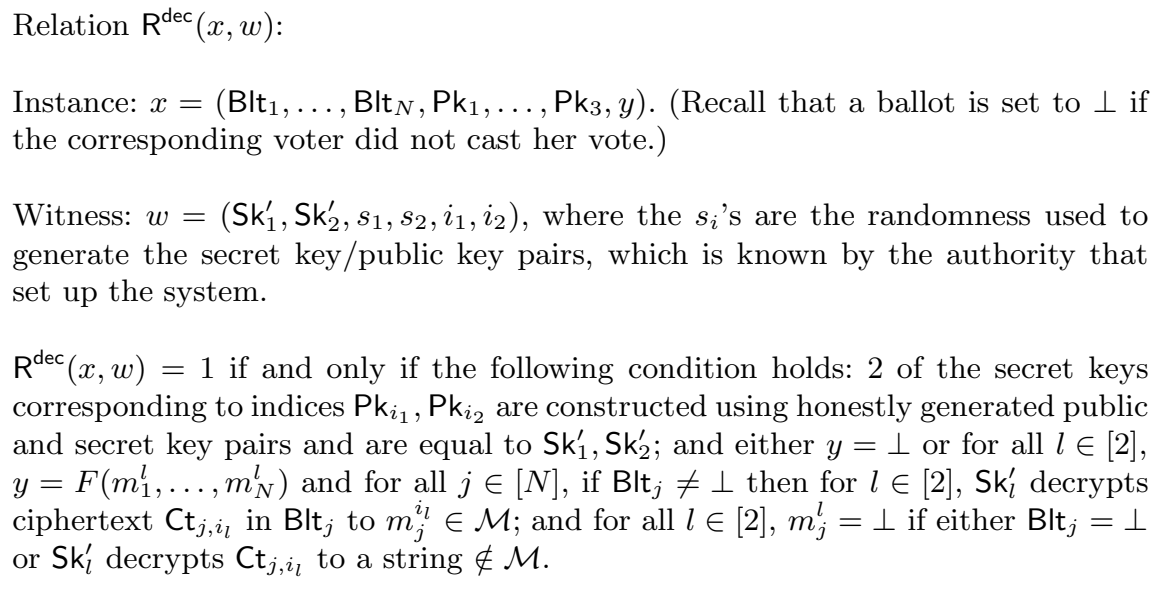

Witness: $w=\left(\mathbf{S k}_{1}^{\prime}, \mathbf{S k}_{2}^{\prime}, s_{1}, s_{2}, i_{1}, i_{2}\right)$, where the $s_{i}$ 's are the randomness used to generate the secret key/public key pairs, which is known by the authority that set up the system.

$\mathrm{R}^{\mathrm{dec}}(x, w)=1$ if and only if the following condition holds: 2 of the secret keys corresponding to indices $\mathrm{Pk}_{i_{1}}, \mathrm{Pk}_{i_{2}}$ are constructed using honestly generated public and secret key pairs and are equal to $\mathrm{Sk}_{1}^{\prime}, \mathrm{Sk}_{2}^{\prime}$; and either $y=\perp$ or for all $l \in[2]$, $y=F\left(m_{1}^{l}, \ldots, m_{N}^{l}\right)$ and for all $j \in[N]$, if $\mathrm{Blt}_{j} \neq \perp$ then for $l \in[2], \mathrm{Sk}_{l}^{\prime}$ decrypts ciphertext $\mathrm{Ct}_{j, i_{l}}$ in $\mathrm{Blt}_{j}$ to $m_{j}^{i_{l}} \in \mathcal{M}$; and for all $l \in[2], m_{j}^{l}=\perp$ if either $\mathrm{Blt}_{j}=\perp$ or $\mathrm{Sk}_{l}^{\prime}$ decrypts $\mathrm{Ct}_{j, i_{l}}$ to a string $\notin \mathcal{M}$.

Fig. 1. Relation $\mathrm{R}^{\mathrm{dec}}$.

Note that the proof $\gamma$ can be computed using as witness the randomness used to compute the public and secret key pairs. Finally, the algorithm outputs the pair $(y, \gamma)$. The tally verification algorithm verifies $(y, \gamma)$ by using the verification algorithm of the NIWI system.

Weak Verifiability of the Construction Weak verifiability (cf. Def. 3) requires that, given a public key and a set of messages decrypted from the ballots, the authority cannot output a pair $(y, \gamma), y \neq \perp$, such that $y$ is an incorrect tally, but $\gamma$ is accepted by the tally verification algorithm. However, the authority is able to claim that $y=\perp$ even if that is not the correct tally. The construction described above suffers from this problem.

We give a detailed proof that our construction fulfills the weak verifiability property in Theorem 1. In the following, we explain why our construction above fulfills the two conditions required by the weak verifiability property. First, we 
show that it fulfills the first condition. The first condition states that, if each ballot and the proof of correctness of the tally issued by the authority are verified successfully by the respective algorithms, then each ballot $C_{i}$ (possibly computed on input a maliciously generated public key) must be associated with a unique message $m_{i} \in \mathcal{M} \cup\{\perp\}$, and the result $y$ claimed by the authority equals $F\left(m_{1}, \ldots, m_{n}\right)$.

We use a contradiction to show that our construction fulfills the first condition. Let us assume that there exist two results $y_{0}, y_{1} \neq \perp$ such that $y_{0} \neq y_{1}$, and two proofs $\gamma_{0}, \gamma_{1}$ that are accepted by the tally verification algorithm. By the unique secret key property, the decryption of the ciphertexts in the ballots produces a unique result. By the pigeon principle, there exists one index $i^{\star} \in[3]$ used by both proofs. Therefore, it must be the case that either $y_{0}=y_{1}=\perp$ or $y_{0}$ and $y_{1}$ are equal to the evaluation of the tally function $F$ on input the messages obtained by decrypting the ciphertexts. Consequently, $y_{0}, y_{1} \neq \perp$ such that $y_{0} \neq y_{1}$ is a contradiction.

The second condition requires that, even when the adversary generates the public key, if honest voters cast a ballot that is accepted by the ballot verification algorithm, then the ballot has to be "counted". We recall that an honest ballot for the $j$-th voter consists of three ciphertexts that encrypt the same message $m$. The perfect soundness of the NIWI ensures that the public key for the PKE scheme is honestly generated. The perfect correctness of the PKE scheme ensures that a ballot that encrypts $m$ will be decrypted to $m$. Therefore, if the claimed tally $y$ does not equal $\perp, y$ has to be in the range of the function $F$ restricted to $m$ at index $j$.

Weak Privacy of the Construction We explain how we prove that our construction fulfills the weak privacy property. The proof consists of a sequence of hybrid experiments GM84, which are summarized in Table 1.5 .

Table 1. Sequence of hybrid games to prove fulfillment of the weak privacy property.

\begin{tabular}{|c|c|c|c|c|}
\hline $\operatorname{Exp}$ & $\left(\mathrm{Ct}_{j, 1}, \mathrm{Ct}_{j, 2}, \mathrm{Ct}_{j, 3}\right)$ & Sk index & $\gamma$ & Security \\
\hline$H_{1}$ & $\left(m_{0, j}, m_{0, j}, m_{0, j}\right)$ & $(1,2,3)$ & $\mathrm{R}$ & - \\
\hline$H_{2}$ & $\left(m_{0, j}, m_{0, j}, m_{1, j}\right)$ & $(1,2,3)$ & $\mathrm{R}$ & IND-CPA \\
\hline$H_{3}$ & $\left(m_{0, j}, m_{0, j}, m_{1, j}\right)$ & $(1,2,3)$ & $\mathrm{T}$ & WI \\
\hline$H_{4}$ & $\left(m_{0, j}, m_{1, j}, m_{1, j}\right)$ & $(1,2,3)$ & $\mathrm{T}$ & IND-CPA \\
\hline$H_{5}$ & $\left(m_{0, j}, m_{1, j}, m_{1, j}\right)$ & $(1,2,3)$ & $\mathrm{T}$ & WI \\
\hline$H_{6}$ & $\left(m_{1, j}, m_{1, j}, m_{1, j}\right)$ & $(1,2,3)$ & $\mathrm{T}$ & IND-CPA \\
\hline$H_{7}$ & $\left(m_{1, j}, m_{1, j}, m_{1, j}\right)$ & $(1,2,3)$ & $\mathrm{R}$ & WI \\
\hline
\end{tabular}

For simplicity, in this sketch we assume that the adversary submits a challenge that consists of two tuples $\left(m_{0,1}, \ldots, m_{0, N}\right)$ and $\left(m_{1,1}, \ldots, m_{1, N}\right)$, where each of the messages belongs to the message space $\mathcal{M}$. In the table, the first 
column shows the name of the hybrid experiment. The second column shows the three messages that are encrypted in the 3 ciphertexts $\mathrm{Ct}_{j, 1}, \ldots, \mathrm{Ct}_{j, 3}$ contained in the challenge ballot $\mathrm{Blt}_{j}=\left(\mathrm{Ct}_{j, 1}, \ldots, \mathrm{Ct}_{j, 3}\right)$ associated with voter $j$. The text in blue in the "Sk index" column denotes the indices used as witness in the proof $\gamma$. As mentioned above, if such blue indices correspond to the set $\{1,2\}$ (resp. to a set different from $\{1,2\}$ ) we say that the statement or proof is in real mode (resp. trapdoor mode), which we denote by $R$ (resp. $T$ ) in the column $\gamma$. The text in red indicates the difference from the previous hybrid experiment.

The proofs of indistinguishability between the hybrid experiments $H_{1}$ and $H_{2}, H_{2}$ and $H_{3}$, and $H_{3}$ and $H_{4}$ are symmetrical to the proofs of indistinguishability between $H_{4}$ and $H_{5}, H_{5}$ and $H_{6}$, and $H_{6}$ and $H_{7}$. Therefore, it suffices to explain how we prove indistinguishability between the first four hybrid experiments.

Hybrid $H_{1}$ corresponds to the real experiment, except that the challenger sets the bit $b=0$.

In hybrid $H_{2}$, we switch the third message (in red) in any ballot to encrypt $m_{1, j}$. This is possible because the witness used to compute the proof $\gamma$ does not contain the randomness used to compute the third secret key. Thanks to that, we can show indistinguishability between $H_{1}$ and $H_{2}$ by using the IND-CPA property of the PKE scheme.

In hybrid $H_{3}$, the witness used to compute the proof $\gamma$ contains the indices $\{1,3\}$ instead of $\{1,2\}$. Therefore, $\gamma$ is in trapdoor mode. The witnessindistinguishability property of the NIWI allows as to show that $H_{3}$ cannot be distinguished from $\mathrm{H}_{2}$. Note that the result of the decryption does not change thanks to the constraint in the weak privacy definition that $F\left(m_{0,1}, \ldots, m_{0, N}\right)=$ $F\left(m_{1,1}, \ldots, m_{1, N}\right)$ must hold.

In hybrid $H_{4}$, we switch the second message (in red) in any ballot to encrypt $m_{1, j}$. This is possible because the witness used to compute the proof $\gamma$ does not contain the randomness used to compute the second secret key. Thanks to that, we can show indistinguishability between $H_{4}$ and $H_{3}$ by using the IND-CPA property of the PKE scheme.

We remark that, in order to switch the encrypted messages to $m_{1, j}$ 's in every ballot, we use a simple property: the witness of the proof $\gamma$ contains the randomness used to compute two of the secret keys. Thanks to that, we can show indistinguishability between $H_{1}$ and $H_{2}$ and between $H_{3}$ and $H_{4}$ by using the IND-CPA property of the PKE scheme whose randomness is not needed to compute $\gamma$. We point out that, to prove indistinguishability between those hybrid experiments, we need to use $N$ "sub-hybrids". In each "sub-hybrid", we switch the message encrypted in just one ballot.

In Section 4, we present our weakly verifiable eVote in a more detailed manner.

\subsection{Our Fully Verifiable eVote (Sketch)}

The scheme sketched in Section 1.5 suffers from a severe problem: the authority can claim that the tally is $\perp$ when it is not. That is, there can be two tallies 
$y_{0} \neq \perp$ and $y_{1}=\perp$ and two proofs $\gamma_{0}$ and $\gamma_{1}$ such that both proofs are accepted by the tally verification algorithm.

For instance, consider the following case. The ballots submitted by the voters are such that the tally $y_{1}$ obtained by evaluating the tally function on input the messages decrypted from the first ciphertext of each ballot equals the tally $y_{2}$ obtained when using the second ciphertext of each ballot, but differs from the tally $y_{3}$ obtained when using the third ciphertext. Then, by using the indices $(1,2)$, the authority can prove successfully that the result of the election is $y_{1}=$ $y_{2}$, and by using indices $(1,3)$, the authority can claim that the result of the election was $\perp$. The voters do not learn the indices that the authority used in the NIWI proof.

This also allows severe DoS attacks. For example, if just one voter submits a wrong ballot that makes the two tallies $y_{1}$ and $y_{2}$ be different from each other, then an honest authority has to output $\perp$. Furthermore, this scheme only fulfills the weak privacy property, which does not take into account corrupt voters.

Therefore, we propose a scheme that fulfills the (full) verifiability and the (full) privacy properties. This scheme solves the above-mentioned problems in an elegant way. Here we show a sketch of the scheme. In Section 5, we present our (fully) verifiable eVote in a more detailed manner.

Sketch of the Construction In addition to the three public keys of the PKE scheme, the public key of the authority contains a perfectly binding commitment $Z$ to the bit 1, i.e., the public key is $\mathrm{Pk}=\left(\mathrm{Pk}_{1}, \mathrm{Pk}_{2}, \mathrm{Pk}_{3}, Z\right)$, where $Z=\operatorname{Com}(1)$.

A ballot consists of three ciphertexts, which are computed as in the weakly verifiable scheme, and of a proof that either the three ciphertexts encrypt the same message in the message space $\mathcal{M} \cup\{\perp\}$ or $Z$ is a commitment to 0 . Formally, the ballot contains a NIWI proof for the relation in Fig. 2 ,

The ballot verification algorithm runs the verification algorithm for the NIWI proof system for the relation $R^{\text {enc,full }}$. (We recall that the ballot verification algorithm of our weakly verifiable eVote accepts any ballot.) The tally evaluation algorithm is the same as in our weakly verifiable eVote.

The tally verification algorithm also follows the one of the weakly verifiable eVote with the following modification. If either (1) not all inputs are $\perp$ and $y=\perp$, or (2) all inputs are $\perp$ and $y \neq \perp$, the tally verification algorithm outputs $\perp$.

We explain the reason for this modification. First, note that, in the (fully) verifiable scheme, the ballots that are rejected by the ballot verification algorithm are replaced by $\perp$ as input to the tally evaluation algorithm. We recall that, in the weakly verifiable scheme, the tally evaluation algorithm is run on input $\perp$ only when voters do not send any ballot.

Our tally functions must fulfill a very natural property: $F\left(m_{1}, \ldots, m_{N}\right)=\perp$ iff $m_{1}=\perp, \ldots, m_{N}=\perp$ (cf. Def. 2). That is, if at least one message is valid, then it has to be "counted".

As we show below, if the public key is honestly generated, the tally evaluation algorithm never returns $\perp$ on input a tuple of possibly dishonest ballots. 
Relation $\mathrm{R}^{\text {enc, full }}(x, w)$ :

Instance: $x \triangleq\left(j, \mathrm{Ct}_{1}, \ldots, \mathrm{Ct}_{3}, \mathcal{E} \cdot \mathrm{Pk}_{1}, \ldots, \mathcal{E} \cdot \mathrm{Pk}_{3}, Z\right)$.

Witness : $w \triangleq\left(m, r_{1}, \ldots, r_{3}, u\right)$, where the values $r_{l}$ 's are the random values used to encrypt the ciphertexts $\mathrm{Ct}_{l}$ 's and $u$ is the random value used to compute the commitment $Z$.

$\mathrm{R}^{\text {enc,full }}(x, w)=1$ if and only if either of the following two conditions hold:

1. Real mode. All 3 ciphertexts $\left(\mathrm{Ct}_{1}, \ldots, \mathrm{Ct}_{3}\right)$ encrypt the same message in $\mathcal{M} \cup\{\perp\}$.

\section{OR}

2. Trapdoor mode. $Z$ is a commitment to 0 .

Fig. 2. Relation $\mathrm{R}^{\mathrm{enc}, \text { full }}$.

Therefore, except for the case that all the ballots are invalid, a tally $y=\perp$ may only occur if the authority acted dishonestly and, consequently, the tally verification algorithm should not accept $y=\perp$.

(Full) Verifiability of the Construction We show that our scheme fulfills the (full) verifiability property. This property consists of two conditions described in Section 1.4 and defined in Def. 3

First, we show that our scheme fulfills the first condition. (A detailed proof is given in Theorem 4.) This condition requires that the authority cannot output two tallies $y_{1}, y_{2}$ such that $y_{1} \neq y_{2}$ and two proofs $\gamma_{1}$ and $\gamma_{2}$ that are accepted by the tally verification algorithm.

Our tally verification algorithm only accepts a tally $y=\perp$ when all the ballots are invalid. Therefore, (1) the authority is not able to wrongly claim that a tally is $\perp$. Furthermore, as in our weakly verifiable eVote, (2) the authority cannot output two tallies $y_{1}, y_{2}$ such that $y_{1} \neq y_{2}, y_{1}, y_{2} \neq \perp$ along with proofs $\gamma_{1}$ and $\gamma_{2}$ that are accepted by the ballot verification algorithm. Therefore, (1) and (2) imply that the authority cannot output two tallies $y_{1}, y_{2}$ such that $y_{1} \neq y_{2}$.

We show now that the second condition also holds. First, we note that the authority can only create a dishonest public key by setting the commitment dishonestly. The reason is that the authority has to prove that the public key of the PKE scheme is honestly generated. Therefore, the perfect correctness of the NIWI and of the PKE scheme guarantee that an honestly computed ballot

${ }^{4}$ Here, "honestly computed ballot" just means that it is computed by the voter using the Cast algorithm on input the public key of the authority, which could be honestly 
for the $j$-th voter that encrypts message $m$ will always be "counted", i.e., for any $(y, \gamma)$ pair that is accepted by the tally verification algorithm, $y$ will be compatible with $m$ at index $j$ according to Def. 1 .

(Full) Privacy of the Construction We show now that our scheme fulfills the (full) privacy property. Here we summarize the proof. In Section 5.2. we describe the proof in detail. We stress that, for privacy to hold, the authority must be honest and thus the public key is honestly generated.

In the security proof, we consider a sequence of hybrid experiments. First, we define an experiment $H^{Z}$ in which the commitment in the public key is a commitment to 0 . We show that $H^{Z}$ is indistinguishable from the real experiment under the computationally hiding property of the commitment.

Second, we define an event $E^{1}$ in experiment $H^{Z}$. In $E^{1}$, the adversary submits a ballot that is accepted by the ballot verification algorithm but, when decrypting the three ciphertexts in the ballot, the three decrypted messages in $\mathcal{M} \cup\{\perp\}$ are not equal. We show that the probability of $E^{1}$ is negligible under the computationally hiding property of the commitment. More concretely, we show that, if $E^{1}$ occurs with non-negligible probability, then the adversary can be used to distinguish a commitment to 0 from a commitment to 1 . We note that, if $Z$ is a commitment to 1 , the perfect soundness of the NIWI guarantees that the adversary can never submit an ill-formed ballot that is accepted by the ballot verification algorithm.

The next hybrid experiments are similar to the ones used in the security proof of the weakly verifiable scheme. Thanks to the hybrid experiment $H^{Z}$, we can still show indistinguishability between those hybrid experiments by using the IND-CPA property of the PKE scheme. The reason is that, thanks to $H^{Z}$, the NIWI proof in the ballots can be a proof that the commitment in the public key is a commitment to 0 . Therefore, we avoid the computation of a proof that shows that the three ciphertexts encrypt the same message, which allows us to switch the message encrypted in one of the ciphertexts and prove indistinguishability by using the IND-CPA assumption.

There is one difference between the hybrid experiments in the weakly verifiable scheme and in the (fully) verifiable scheme. Namely, in the (fully) verifiable scheme, we have to handle possibly dishonest ballots. In particular, we have to guarantee that, when we switch the indices used as witness for the NIWI proof of tally correctness, the tally does not change. To illustrate this issue, suppose that, in an adversarial ballot, the first two ciphertexts encrypt the same message $x$ but the third one encrypts a different message $z$. Then the tally computed by the secret keys for indices $\{1,2\}$ could differ from the one computed with secret keys for indices $\{2,3\}$. In that case, we cannot prove indistinguishability

or dishonestly created. By design of our construction, an honestly generated ballot computed on input an honestly created public key has the same distribution of an honestly created ballot computed on input any possibly dishonest public key whenever the authority is able to compute proofs of tally correctness that are accepted by the tally verification algorithm. 
between a hybrid experiment where the NIWI witness comprises $\mathbf{S k}_{1}, \mathrm{Sk}_{2}$ and a hybrid experiment where the NIWI witness comprises $\mathbf{S k}_{2}, \mathbf{S k}_{3}$.

To solve this issue, we show that event $E^{1}$ occurs with negligible probability. Therefore, it is sufficient to analyze the advantage of the adversary in the hybrid experiments conditioned on the occurrence of $\bar{E}^{1}$ (i.e., the complement of $E^{1}$ ).

More concretely, the sequence of hybrid experiments after $H^{Z}$ is as follows. We recall that the adversary sends two tuples $V_{0}=\left(m_{0,1}, \ldots, m_{0, N}\right)$ and $V_{1}=$ $\left(m_{1,1}, \ldots, m_{1, N}\right)$, and a set $S \subset[N]$ that contains the indices of the strings of arbitrary ballots.

- Hybrid experiment $H_{1}$ is equal to the experiment $H^{Z}$, except that the challenger sets the bit $b=0$.

- Hybrid experiment $H_{2}$ switches the message encrypted in the third ciphertext in any ballot to encrypt $m_{1, j}$ instead of $m_{0, j}$. More in detail, for $k=0$ to $N$, we define a sequence of hybrid experiments $H_{2}^{k} . H_{2}^{k}$ is identical to $H_{1}$, except that, for all $j=1, \ldots, k$ such that $j \notin S$, the challenger computes the third ciphertext of the ballot on input $m_{1, k}$. Therefore, $H_{2}^{0}$ is identical to $H_{1}$, while $H_{2}^{N}$ is identical to $H_{2}$. We show that $H_{2}^{k}$ and $H_{2}^{k+1}$ are indistinguishable thanks to the IND-CPA property of the PKE scheme.

- Hybrid experiment $H_{3}$ is identical to experiment $H_{2}$, except that the challenger computes the NIWI proof $\gamma$ on input a witness that contains indices $(1,3)$ and secret keys $\mathbf{S k}_{1}, \mathbf{S k}_{3}$, instead of indices $(1,2)$ and secret keys $\mathbf{S k}_{1}$, $\mathrm{Sk}_{2}$. We show that $H_{3}$ and $H_{2}$ are indistinguishable thanks to the witnessindistinguishability property of the NIWI proof. Because $\bar{E}^{1}$ occurs with overwhelming probability, any ballot in $S$ is either replaced by $\perp$, if the ballot verification algorithm does not accept it, or decrypted to the same value in $\mathrm{H}_{2}$ and $\mathrm{H}_{3}$. Therefore, the tally evaluation algorithm outputs the same tally in $\mathrm{H}_{2}$ and $\mathrm{H}_{3}$.

- Hybrid experiment $H_{4}$ is identical to $H_{3}$, except that the second ciphertext in any ballot encrypts $m_{1, j}$ instead of $m_{0, j}$. More in detail, for $k=0$ to $N$, we define a sequence of hybrid experiments $H_{4}^{k}$. $H_{4}^{k}$ is identical to $H_{3}$, except that, for all $j=1, \ldots, k$ such that $j \notin S$, the challenger computes the second ciphertext of the ballot on input $m_{1, k}$. Therefore, $H_{4}^{0}$ is identical to $H_{3}$, while $H_{4}^{N}$ is identical to $H_{4}$. We show that $H_{4}^{k}$ and $H_{4}^{k+1}$ are indistinguishable thanks to the IND-CPA property of the PKE scheme.

The remaining hybrid experiments are symmetrical to the ones described above. In $H_{5}$, the witness used to compute the NIWI proof contains the indices $(2,3)$ and secret keys $\mathrm{Sk}_{2}, \mathrm{Sk}_{3}$, and indistinguishability between $H_{5}$ and $\mathrm{H}_{4}$ follows from the witness-indistinguishability property of the NIWI proof. In $H_{6}$, the first ciphertext of each ballot encrypts $m_{1, j}$ instead of $m_{0, j}$ and indistinguishability between $H_{6}$ and $H_{5}$ follows from the IND-CPA property of the PKE scheme. Finally, in $H_{7}$ the witness used to compute the NIWI proof contains the indices $(1,2)$ and secret keys $\mathbf{S k}_{1}, \mathbf{S k}_{2}$, and indistinguishability between $H_{7}$ and $H_{6}$ follows from the witness-indistinguishability property of the NIWI proof.

We would like to remark the subtle difference between ill-formed and invalid ballots. An ill-formed ballot is a ballot that is not in the range of the cast algo- 
rithm. However, an ill-formed ballot could be valid in the sense that, along with other (possibly ill- or well- formed) $N-1$ ballots, the authority obtains a tally, i.e., the tally obtained when decrypting the first and the second ciphertext in the ballots is the same. An ill-formed ballot can be computed when the commitment in the public is computed dishonestly.

The event $\bar{E}^{1}$ may occur even if the adversary submits an ill-formed ballot that is accepted by the ballot verification algorithm. In fact, if a (non-honestly computed) ballot is formed by strings that are not in the ciphertext space of the encryption algorithm of the PKE, but decryption of those strings outputs the same message, such a ballot is not considered invalid.

Note also that the proof of well-formedness of the ballots states that the encrypted messages may be equal to $\perp$. Ballots that encrypt $\perp$ are blank ballots. We consider tally functions in which the symbol $\perp$ indicates a blank vote. For example, in case of an eVote for the sum function in which $\perp$ is counted as 0 , an adversary should not be able to distinguish three ballots that encrypt $(1,1, \perp)$ from three ballots that encrypt $(1, \perp, 1)$.

\section{7 eVote with Multiple Authorities and Threshold Privacy}

In this section, we sketch how to generalize our (fully) verifiable construction to fit a model with multiple authorities. In this model, the tally evaluation algorithm is run by a set of authorities. The privacy property must hold if not all the authorities are corrupt. Our generalized scheme guarantees a statistical verifiability property (see below), which assumes that there is at least one honest voter.

First, we note that the multi-string model of Groth and Ostrovsky GO14 does not provide a solution to this problem. The multi-string model assumes that the majority of the parties that set up the CRSs are honest. It does not guarantee soundness, which would provide verifiability in our application, when all those parties, which would be the authorities in our application, are corrupt. In the multi-string model, there is a trade-off between soundness and zero-knowledge. Namely, soundness could hold when all the authorities are corrupt, but then zero-knowledge does not hold. Zero-knowledge is guaranteed only when there is a majority of honest authorities. In contrast, our generalized scheme fulfills the privacy property when at least one authority is honest.

Sketch of the Construction Our generalized construction works for tally functions that can be represented as polynomials. Such tally functions comprise many functions of interest for e-voting. For simplicity, henceforth we only consider the case of the sum function with a binary message space. The general case follows by using Lagrange's polynomial interpolation.

Consider the sum function over a set of integers $S^{k}$, which we specify later. Consider $m$ authorities. Each authority $k \in[m]$ publishes a public key that consists of the public key of our (fully) verifiable eVote and, in addition, a commitment $\mathrm{com}_{k}$ to a tuple of $N$ 0's. 
A ballot $\mathrm{Blt}_{j}$ for the $j$-th voter consists of the ballots $\left(\mathrm{Blt}_{j, 1}, \ldots, \mathrm{Blt} \mathrm{t}_{j, m}\right)$. For a vote $v_{j}$, each voter computes $m$ shares $v_{j, 1}, \ldots, v_{j, m}$ whose sum is $v_{j}$. (Later we describe how the shares are computed in order to preserve privacy.) The ballot $\mathrm{Blt}_{j, k}$ for the $k$-th authority is computed following the cast algorithm of the (fully) verifiable scheme on input the share $v_{j, k}$. In addition, the voter adds a NIWI proof that either (the real statement) for all $k \in[m]$, Blt ${ }_{j, k}$ encrypts a number in $S^{k}$ such that the sum of the encrypted numbers is in $\{0,1\}$ (for simplicity, here we do not consider messages equal to $\perp$ ) OR (the trapdoor statement) for all $k \in[m], \operatorname{com}_{k}$ is a commitment to a tuple $\left(z_{1}, \ldots, z_{N}\right)$ such that $z_{j}$ is equal to the tuple $\left(\mathrm{Blt}_{j, 1}, \ldots, \mathrm{Blt}_{j, m}\right)$.

For each $k \in[m]$, the $k$-th authority computes the tally $y_{k}$ as in the (fully) verifiable scheme. The proof of correctness of the tally is a proof for the following modified relation: either (the real statement) the witness satisfies the relation $\mathrm{R}^{\text {dec,full }}$ of the (fully) verifiable scheme and $\operatorname{com}_{k}$ is a commitment to 0 OR (the trapdoor statement) $\mathrm{com}_{k}$ is a commitment to a tuple $\left(z_{1}, \ldots, z_{N}\right)$ such that $z_{j}=\mathrm{Blt}_{j}$ for all $j \in[N]$, where $\mathrm{Blt}_{1}, \ldots, \mathrm{Blt}_{N}$ are the $N$ ballots published on the public bulletin board.

Finally, the tally is computed by summing the tallies $y_{k}$ 's output by each of the authorities to obtain $y$. We give more details below.

To support functions represented as polynomials, the following modifications should be applied. To compute the shares $v_{j, 1}, \ldots, v_{j, m}$, the voter $j$ chooses a polynomial $p_{j}$ of degree $m-1$ such that $p_{j}(0)$ equals her vote $v_{j}$. The shares are the evaluation of $p_{j}$ on input $1, \ldots, m$. The tally is computed by using Lagrange interpolation.

Verifiability of the Construction We analyze now the verifiability of our generalized construction. If the commitments in the public key are computed honestly, we can show that the generalized construction fulfills the verifiability property by using the same arguments given for our construction with one authority.

Consider that w.l.o.g the $k$-th authority outputs a commitment $\operatorname{com}_{k}$ that does not commit to a tuple of 0 's. If at least one voter $j$ is honest, the probability that this voter outputs a ballot $\mathrm{Blt}_{j}$ such that $\operatorname{com}_{k}$ is a commitment to a tuple $\left(z_{1}, \ldots, z_{N}\right)$ and $z_{j}=\mathrm{Blt}_{j}$ is negligible over the random coins of the $j$-th voter. Therefore, assuming that there is at least one honest voter, the authorities can compute proofs of tally correctness by using the witness for the "trapdoor statement" in the relation only with negligible probability. Similarly, assuming that there is at least one honest voter, the voters can compute proofs of ballot correctness by using the witness for the "trapdoor statement" only with negligible probability over the random coins of the honest voters. In conclusion, the generalized construction fulfills (a statistical variant of) the verifiability property thanks to the verifiability of the (fully) verifiable eVote in Section 5 and to the fact that, in real mode, the sum of the messages encrypted in a ballot is equal to a number in $\{0,1\}$. 
Privacy of the Construction We use a selectively-secure model CHK04 for our definition of privacy. In the game between the challenger and the adversary, the adversary has to declare its challenge at the outset of the game before receiving the public keys of the authorities. The adversary is allowed to receive the secret keys of all except one authority.

We show that our generalized construction fulfills this definition of privacy. First, we define the sets $S^{k}$ and a method for computing the shares $v_{j, 1}, \ldots, v_{j, m}$ for a vote $v_{j}$. This method must guarantee that any subset of $m-1$ authorities does not get any information about $v_{j}$. For simplicity, consider that $m=2$. Then, the sets $S^{1}=S^{2}=S$ are equal by definition to $\{-p, \ldots, p\}$, where $p$ is a number of size super-polynomial in the security parameter. The message space of the PKE scheme must comprise numbers between $-N p$ and $N p$. To encrypt 0 (resp. 1), the voter chooses a random number $v_{1}$ in $S$ and sets $v_{2}$ to $-v_{1}$ (resp. $\left.-v_{1}+1\right)$. It is easy to see that, except when either $v_{1}$ or $v_{2}$ equal $-p$, any value of $v_{2}$ (resp. $\left.v_{1}\right)$ can correspond to $v_{1}=-v_{2}\left(\right.$ resp. $\left.v_{2}=-v_{1}\right)$ if the voter cast a vote for 0 or to $v_{1}=-v_{2}+1$ (resp. $v_{2}=-v_{1}+1$ ) if the voter cast a vote for 1 . The case in which either $v_{1}$ or $v_{2}$ equal $-p$ occurs with negligible probability, which is guaranteed by choosing $p$ to be super-polynomial in the security parameter. Consequently, each authority does not get any information on the vote $v_{j}$. This method can be generalized to the case $m>2$. We skip the details.

Because the adversary receives the public keys after sending the challenge, in the security proof we can define a hybrid experiment where the commitments in the public key commit to ballots $\left(\mathrm{Blt}_{1} \ldots, \mathrm{Blt} \mathrm{t}_{N}\right)$ computed on input the challenge messages. Like in the reduction of Section [5.2, we prove that the probability that the adversary submits an ill-formed ballot that is accepted by the ballot verification algorithm is negligible by using the computationally hiding property of the commitment scheme.

In the next hybrid experiments, the NIWI proofs of ballot correctness and of tally correctness can be computed by using the witness for the trapdoor statement, i.e., the randomness used to compute the commitments. Thanks to that, we are able to compute ballots where not all the ciphertexts encrypt the same message. This allows us to switch the message encrypted in one of the ciphertexts of the ballots and prove indistinguishability between the experiments by using the IND-CPA property of the PKE scheme.

To prove that our scheme fulfills a definition for privacy in a non-selective (i.e., full) security model, one can use complexity leveraging arguments. Such arguments can profit from the fact that, in our formulation, we required the number of voters $N$ and the size of the message space to be independent of the security parameter. This allows the challenger to just guess the challenge messages in advance with constant probability. This requirement can be weakened to the case of $N$ and size of message space logarithmic in the security parameter. We leave open how to achieve full security without complexity leveraging.

Note that we do not require any interaction between the authorities. The public keys of the authorities are completely independent from each other. Moreover, the authorities do not need any coordination (e.g., to run sequentially), i.e., the 
tally can be computed and publicly verified from the output of each authority individually. Thus, our techniques completely diverge from previous approaches to the problem.

\subsection{On The Reusability of the Public Parameters}

Our definition of verifiability does not prevent the following undesirable case. Consider an ill-formed ballot Blt ${ }_{1}$. Consider other valid ballots $\mathrm{Blt}_{2}, \ldots, \mathrm{Blt}_{N}$ that encrypt respectively $v_{2}, \ldots, v_{N}$. The authority is able to compute a tally $y=F\left(v_{1}, \ldots, v_{N}\right)$ and a valid proof of tally correctness. Consider now other valid ballots $\mathrm{Blt}_{2}^{\prime}, \ldots, \mathrm{Blt}_{N}^{\prime}$ that encrypt $v_{2}^{\prime}, \ldots, v_{N}^{\prime}$. The authority can possibly compute another tally $y^{\prime}=F\left(v_{1}^{\prime}, v_{2}^{\prime}, \ldots, v_{N}^{\prime}\right)$ and another proof of tally correctness. The problem is that the ill-formed ballot $\mathrm{Blt}_{1}$ can be decrypted to more than one message.

This does not contradict our definition because, for $\mathrm{Blt}_{1}, \ldots, \mathrm{Blt} \mathrm{t}_{N}$, there still exist messages $v_{1}, \ldots, v_{N}$ that satisfy the statement of the definition, i.e., given $\mathrm{Pk}$ and $\mathrm{Blt}_{1}, \ldots, \mathrm{Blt}{ }_{N}$, the authority cannot output two different results and two valid proofs of tally correctness for each of them. However, it can occur that for $\mathrm{Pk}, \mathrm{Blt}_{1}, \mathrm{Blt}_{2}^{\prime}, \ldots, \mathrm{Blt}_{N}^{\prime}$, there are different messages that satisfy the definition. We remark that the public key $\mathrm{Pk}$ does not change.

Let us present a concrete example. Consider two 0/1 elections with only 2 voters. A ballot could possibly be reused in the second election, i.e., if the public parameters of the system are reused, the same ballot can be cast again. Given an ill-formed ballot Blt $t_{1}$, there could exist two ballots $B \mathrm{t}_{2}$ and $\mathrm{BIt}_{2}^{\prime}$ such that, in an election with ballots $B \mid t_{1}$ and $B \mid t_{2}$, the result is 2 , and, in a election with ballots $\mathrm{Blt}_{1}$ and $\mathrm{Blt}_{2}^{\prime}$, the result is 0 . This can only happen if the first ballot is "associated" with vote 1 in the first election and with vote 0 in the second election. Therefore, the first and the second elections are incoherent. More undesirable issues would emerge if different tally functions could be computed in different elections carried out with the same parameters and ballots.

A stronger definition could state that, for all $\mathrm{Pk}$ and all Blt $\mathrm{t}_{1}$, there exists $m_{1}$ such that, for all $\mathrm{Blt}_{2}, \ldots, \mathrm{Blt}{ }_{N}$, there exist $m_{2}, \ldots, m_{n}$ such that the authority is only able to output a tally $y=F\left(m_{1}, \ldots, m_{N}\right)$ along with a valid proof of tally correctness. We note that this is a simplification because a general definition should take into account multiple dishonest voters.

Fortunately, in our e-voting model, as well as in other traditional models, the parameters cannot be reused through different elections. Therefore, the abovementioned problem does not occur.

In a stronger model in which the parameters can be reused, our constructions would not be secure. Nevertheless, in our (fully) verifiable construction, the inconsistency of results through different elections would occur only in the case that a malicious authority sets the commitment in the public key dishonestly to 0 , which allows the computation of ill-formed ballots.

This state of affairs could be paralleled to the case of garbled circuits, where the original one-time version Yao86 LP09 can be based on the minimal assumption of existence of one-way functions, whereas the reusable variant $\mathrm{GKP}^{+} 13$ ] 
is known to be implementable only under stronger assumptions. Similarly, in functional encryption, the schemes with bounded security [SS10|GVW12 can be based just on public key encryption, whereas the unbounded secure variants are only known to be implementable under very strong assumptions GGHZ16. For instance, the scheme of Sahai and Seyalioglu [SS10] becomes completely insecure when the adversary can decrypt a ciphertext with two different secret keys, exactly as it occurs for our schemes.

\subsection{Related Work}

Our work is inspired by the work of Badrinarayanan et al. BGJS16, which puts forward the concept of verifiable functional encryption. (We note that the committing IBE of GH07 can be seen as a weaker variant of verifiable identitybased encryption.) Our work shares with BGJS the idea of "engineering" multiple witnesses, which are needed when using NIWI proofs, to enforce privacy in conjunction with verifiability.

Notwithstanding, the constructions are quite different, especially due to the different requirements of functional encryption and e-voting. For instance, in the security definition of functional encryption, the keys are handed to the adversary, so one needs a proof that each secret key and ciphertext is computed correctly. Instead, in our case, the adversary does not see the secret key. We can profit from this fact to just prove that the claimed tally equals the evaluation of the tally function over all ballots.

Such complications in functional encryption introduce a severe limitation: in the security reduction of BGJS, it is fundamental that the public key contain a commitment that in some hybrid experiment is set to the challenge ciphertext. Therefore, it is assumed that the adversary commits to the challenge before receiving the public key, i.e., security is proven in the selective model [CHK04. On the contrary, our constructions are secure in the full (i.e., non-selective) model.

In other respects, in e-voting we face new challenges. In BGJS, the challenger computes the NIWI on input a witness that comprises all the secret keys and proves the well-formedness of all the secret keys except one, but, in addition, proves that all the secret keys decrypt some challenge ciphertext correctly. This is sufficient to use the IND-CPA property of functional encryption to prove indistinguishability between two hybrid experiments where the message encrypted in one of the ciphertexts is switched from $m_{0}$ to $m_{1}$. The reason is that the secret keys are supposed to be for the same function $f$ such that $f\left(m_{0}\right)=f\left(m_{1}\right)$. (More concretely, in the IND-CPA property of functional encryption, the adversary is allowed to receive secret keys for a function $f$ that evaluates both challenge messages to the same value.) Therefore, the secret keys do not allow to distinguish between the two ciphertexts. In our setting, we can only input to the NIWI all the secret keys except one. Otherwise we could not use the IND-CPA property to prove indistinguishability between two hybrid experiments where the encrypted message is switched from $m_{0}$ to $m_{1}$. 
Furthermore, in our (full) privacy definition, we have to handle challenge tuples that contain ill-formed ballots, whereas in verifiable multi-input functional encryption the challenge contains only honestly computed ciphertexts. Therefore, the differences between the two settings make the respective techniques utterly incomparable.

It is tempting to think that the construction of BGJS of multi-input verifiable functional encryption (which extends multi-input functional encryption of Goldwasser et al. $\left[\mathrm{GGG}^{+} 14\right]$ ) can be directly used to construct a verifiable eVote. Though it seems plausible, we did not verify that. However, this would eventually result in a verifiable eVote based on indistinguishability obfuscation $\mathrm{GGH}^{+} 13$, a very strong assumption, and would only be secure in the selective model.

Needless to say, our techniques, as well as the ones of BGJS, owe a lot to the celebrated FLS' OR trick [FLS90]. They can be viewed as a generalization of it.

Kiayias et al. [KZZ15] (see also $\mathrm{CZZ}^{+} 15$ ] for a distributed implementation) put forth a verifiable eVote without trust assumptions that represents a breakthrough along this direction, but diverges from ours in several fundamental aspects:

- It requires interaction between the voters and the board, whereas all our algorithms are non-interactive.

- Receipt-freeness, accountability and degree of dependence on secure channels to distribute vote codes are undetermined issues. In our scheme, voters can verify the election if they just know the ballot they cast. In particular, voters do not need to store the randomness used to compute it, and the authority cannot cheat in the tally process.

- It does not achieve universal verifiability, whereas ours does.

- Its information-theoretical verifiability is parameterized and depends on the number of honest voters, whereas ours is perfect, i.e., the probability of a wrong tally being accepted by the verification algorithm is equal to zero.

- Its privacy can be reduced to group-based assumptions at the cost of using complexity leveraging and assuming sub-exponential security, whereas ours only requires the standard version of Decision Linear assumption with polynomial security 5

- Its definition of verifiability requires an extraction property, whereas ours does not.

Moran and Naor [MN06] construct an universally verifiable e-voting protocol with very strong provable-security properties. However, it assumes either the availability of a "random beacon" that has to be sampled honestly or the soundness of the Fiat-Shamir's heuristic. Therefore, verifiability does not hold unconditionally, i.e., without any assumption (both physical or computational).

\footnotetext{
${ }^{5}$ At some point in the security reduction for our (fully) verifiable eVote, we make use of the fact that the number of voters $N$ is a constant independent of the security parameter that could be viewed as a complexity leveraging trick or as problematic in the case that $N$ be large. But we stress that this is done only for simplicity of exposition and we sketch how the reduction and our results can be generalized even to the case of $N(\cdot)$ function of the security parameter.
} 
We are not aware of other traditional e-voting schemes that achieve perfect verifiability without interaction and without trust assumptions. We refer to $\left.\mathrm{CGK}^{+} 16\right]$ and $\left[\mathrm{BCG}^{+} 15\right]$ for a survey.

We point out that our definition of verifiability is motivated by the guidelines of $\mathrm{CGK}^{+} 16$. In its formalization, our definition is similar to the ones of GIR16], the verifiability for multi-input functional encryption of BGJS and the uniqueness of tally of Bernhard et al. $\left[\mathrm{BCG}^{+} 15\right]$. Anyhow, the latter is formulated to hold only against computationally bounded adversaries and both BGJS16 and Bernhard et al. do not take into account our condition (2) for verifiability 6 See also KRS10 for symbolic approaches to verifiability.

Our privacy notion is inspired by the one of Benaloh [Ben87, which is called "PRIV" in $\mathrm{BCG}^{+} 15$. We reformulate it by using modern terminology and we conveniently modify it to withstand the attacks shown in $\left[\mathrm{BCG}^{+} 15\right]$. We refer to $\left[\mathrm{BCG}^{+} 15\right]$ for a survey on definitions of privacy for e-voting.

Perfect verifiability and perfect correctness seem incompatible with receiptfreeness [BT94|SK95|MH96|MN06|DKR09|CCFG15]. Notwithstanding, we think that it should be possible to define a statistical variant of verifiability achievable without any trust assumptions that could coexist with some form of receiptfreeness. Another possibility could be to resort to some voting server trusted for receipt-freeness but not for privacy, such as the server that re-randomizes the ballots in BeleniosRF of Chaidos, Cortier, Fuchsbauer and Galindo CCFG15. (We note that they also address the problem of authenticity that we neglect.) As it is out of the scope of this work, we deliberately omit receipt-freeness in our treatment.

Recently, Bellare, Fuchsbauer and Scafuro BFS16] started the study of security of NIZKs in face of public parameter subversion. They showed the impossibility of attaining subversion soundness while retaining zero-knowledge, thus justifying our need of sidestepping NIZKs.

\section{Definitions}

Notation. A negligible function $\operatorname{negl}(k)$ is a function that is smaller than the inverse of any polynomial in $k$ (from a certain point and on). We denote by $[n]$ the set of numbers $\{1, \ldots, n\}$. If $S$ is a finite set, we denote by $a \leftarrow S$ the process of setting $a$ equal to a uniformly chosen element of $S$. With a slight abuse of notation, we assume the existence of a special symbol $\perp$ that does not belong to $\{0,1\}^{\star}$.

If $A$ is an algorithm, then $A\left(x_{1}, x_{2}, \ldots\right)$ denotes the probability distribution of the output of $A$ when $A$ is run on input $\left(x_{1}, x_{2}, \ldots\right)$ and randomly chosen coin tosses. Instead, $A\left(x_{1}, x_{2}, \ldots ; r\right)$ denotes the output of $A$ when run on input $\left(x_{1}, x_{2}, \ldots\right)$ and (sufficiently long) coin tosses $r$. All algorithms, unless explicitly noted, are probabilistic polynomial time (PPT) and all adversaries are modeled by non-uniform PPT algorithms.

\footnotetext{
${ }^{6}$ Needless to say, for many applications of multi-input functional encryption, the lack of condition (2) could not pose a threat.
} 
If $A$ is a PPT algorithm, we say that $y \in A(x)$ iff there exists a random value $r$ such that $y=A(x ; r)$; in that case, we say that $y$ is in the range of $A(x)$. If $E$ is an event in a probability space, $\bar{E}$ denotes its complement.

The following definition is used in the definition of verifiability. Essentially, it states that a tally $y$ is compatible with votes $z_{1}, \ldots, z_{k}$ if the latter values are in its pre-image.

Definition 1 Given a function $F\left(x_{1}, \ldots, x_{n}\right): A^{n} \rightarrow B$, we say that a value $y \in$ $B$ is compatible with $z_{1}, \ldots, z_{k} \in A$ at indices $i_{1}, \ldots, i_{k} \in[N]$ if $y$ is in the range of the restriction $F_{\mid C_{z_{1}, \ldots, z_{k}, i_{1}, \ldots, i_{k}}}$ of $F$ to $C_{z_{1}, \ldots, z_{n}, i_{1}, \ldots, i_{n}} \triangleq\left\{\left(x_{1}, \ldots, x_{n}\right) \mid \forall j \in\right.$ $\left.[k], x_{i_{j}}=z_{j}\right\}$.

\section{$2.1 \quad$ E-Voting Schemes}

An e-voting scheme (eVote, in short) is parameterized by the tuple $(N, \mathcal{M}, \Sigma, F)$. The natural number $N>0$ is the number of voters. The set $\mathcal{M}$ is the domain of valid votes. The set $\Sigma$ is the range of possible results. The function $F:(\mathcal{M} \cup$ $\{\perp\})^{N} \rightarrow \Sigma \cup\{\perp\}$ is the tally function. We allow the tally function to take as input the special symbol $\perp$, which denotes either an abstention, an invalid ballot or a blank vot 7 , and to output $\perp$ to indicate an error. We require that the tally function outputs an error on input a sequence of strings iff all the strings are equal to $\perp$. Formally, the tally function is defined as follows.

Definition 2 [Tally function] A function $F$ is a tally function if there exists a natural number $N>1$, and sets $\mathcal{M}, \Sigma \subset\{0,1\}^{\star}$ such that the domain of $F$ is $\mathcal{M} \cup\{\perp\}$, the range is $\Sigma \cup\{\perp\}$ and for all strings $m_{1}, \ldots, m_{N} \in \mathcal{M} \cup\{\perp\}$, it holds that $F\left(m_{1}, \ldots, m_{N}\right)=\perp$ iff $m_{1}=\perp, \ldots, m_{N}=\perp$.

Before defining formally an eVote, we explain how its algorithms are used to conduct an election.

The voting ceremony. The voting ceremony occurs as follows.

- Setup phase. An authority (also called voting authority or election authority) uses algorithm Setup to compute a public key Pk and a secret key Sk.

- Voting phase. Each of the $N$ voters runs an algorithm Cast on input the voter identifier $j \in[N]$, the public key Pk and a vote $v \in \mathcal{M}$ to compute a ballot Blt. The voter sends Blt to an append-only public bulletin board (PBB).

- Tallying phase. The well-formedness of each ballot Blt published in the PBB can be publicly verified by means of an algorithm VerifyBallot. If the ballot is invalid, a new row in which the ballot is replaced by $\perp$ is appended to the PBB. Later, only the new row is used. If a voter did not cast a vote, $\perp$ is appended to the PBB.

\footnotetext{
${ }^{7}$ We note that our tally function can be made more general by assigning different symbols to an abstention, to an invalid ballot and to a blank vote.
} 
The authority runs evaluation tally algorithm EvalTally on input the public key, the secret key, and $N$ strings that represent either ballots or $\perp$ symbols appended to the PBB. EvalTally outputs the tally, i.e., the result of the election, and a proof of tally correctness. The tally equals the special symbol $\perp$ to indicate an error.

- Verification phase. Algorithm VerifyTally takes as input the public key, a tuple of $N$ strings that represent either ballots or the special symbol $\perp$, the tally and the proof of tally correctness. VerifyTally outputs a value in $\{\mathrm{OK}, \perp\}$.

Each participant, not necessarily a voter, can verify the correctness of the result of the election as follows. First, verify whether the ballots cast by the voters are valid using the VerifyBallot algorithm. Check whether the authority replaced with $\perp$ only the invalid ballots. Assign $\perp$ to any voter who did not cast her vote. After that, run the VerifyTally algorithm on input the public key, the $N$ strings that represent either ballots or the special symbol $\perp$, the tally and the proof of tally correctness.

Definition 3 [E-voting Scheme] A $(N, \mathcal{M}, \Sigma, F)$-e-voting scheme EVOTE for number of voters $N>1$, domain of valid votes $\mathcal{M}$, range of possible results $\Sigma$ and tally function $F:(\mathcal{M} \cup\{\perp\})^{N} \rightarrow \Sigma \cup\{\perp\}$ is a tuple

$$
\text { EVOTE } \triangleq(\text { Setup, Cast, VerifyBallot, EvalTally, VerifyTally) }
$$

of 5 PPT algorithms, where VerifyBallot and Verify Tally are deterministic, that fulfill the following syntax:

1. Setup $\left(1^{\lambda}\right)$ : on input the security parameter in unary, it outputs the public key $\mathrm{Pk}$ and the secret key Sk.

2. Cast $(\mathrm{Pk}, j, v)$ : on input the public key $\mathrm{Pk}$, the voter identifier $j \in[N]$, and a vote $v \in \mathcal{M}$, it outputs a ballot Blt.

3. VerifyBallot(Pk, $j, \mathrm{Blt})$ : on input the public key $\mathrm{Pk}$, the voter identifier $j \in[N]$ and a ballot Blt, it outputs a value in $\{\mathrm{OK}, \perp\}$.

4. EvalTally $\left(\mathrm{Pk}, \mathrm{Sk}, \mathrm{Blt} \mathrm{t}_{1}, \ldots, \mathrm{Blt}_{N}\right)$ : on input the public key $\mathrm{Pk}$, the secret key Sk, and $N$ strings that are either ballots or the special symbol $\perp$, it outputs the tally $y \in \Sigma \cup\{\perp\}$ and a proof $\gamma$ of tally correctness.

5. VerifyTally $\left(\mathrm{Pk}, \mathrm{Blt}_{1}, \ldots, \mathrm{Blt}_{N}, y, \gamma\right)$ : on input the public key $\mathrm{Pk}, N$ strings that are either ballots or the special symbol $\perp$, a tally $y \in\{0,1\}^{\star} \cup\{\perp\}$ and a proof $\gamma$ of tally correctness, it outputs a value in $\{\mathrm{OK}, \perp\}$.

An eVote must satisfy the following correctness, verifiability, and privacy properties. We also define the weak verifiability and weak privacy properties. A weakly verifiable eVote must satisfy correctness, weak verifiability and weak privacy.

\section{Correctness and verifiability}

- (Perfect) Correctness. We require the following conditions (1) and (2) to hold. 
1. Let Abst be a special symbol not in $\mathcal{M} \cup\{\perp\}$ that denotes that a voter did not cast her vote 8 For all $\mathrm{Pk} \in \operatorname{Setup}\left(1^{\lambda}\right)$, all $m_{1}, \ldots, m_{N} \in \mathcal{M} \cup$ $\{\perp, \mathrm{Abst}\}$, all $\left(\mathrm{Blt}_{j}\right)_{j=1}^{N}$ such that for all $j \in[N], \mathrm{Blt}_{j}=\perp$ if $m_{j}=$ Abst, $\mathrm{Blt}_{j} \in \operatorname{Cast}\left(\mathrm{Pk}, j, m_{j}\right)$ if $m_{j} \in \mathcal{M}$ and $\mathrm{Blt}_{j} \in \operatorname{Cast}(\mathrm{Pk}, j, \perp)$ otherwise, the following two conditions (a) and (b) hold:

(a) For all $j \in[N]$, if $m_{j} \neq$ Abst then VerifyBallot $\left(\mathrm{Pk}, j, \mathrm{Blt}_{j}\right)=\mathrm{OK}$.

(b) if $(y, \gamma) \triangleq$ EvalTally $\left(\mathrm{Pk}, \mathrm{Blt}_{1}, \ldots, \mathrm{Blt}_{N}\right)$ then it holds that: $y=F\left(m_{1}, \ldots, m_{N}\right)$ and VerifyTally $\left(\mathrm{Pk} \mathrm{Blt}_{1}, \ldots, \mathrm{Blt}_{N}, y, \gamma\right)=\mathrm{OK}$.

2. For all $\mathrm{Pk} \in \operatorname{Setup}\left(1^{\lambda}\right), \mathrm{Blt} t_{1}, \ldots, \mathrm{Blt}_{N} \in\{0,1\}^{\star} \cup\{\perp\}$, if $S \triangleq\left\{j \mid \mathrm{Blt}_{j} \neq\right.$ $\perp \wedge$ VerifyBallot $\left.\left(\mathrm{Pk}, j, \mathrm{Blt}{ }_{j}\right)=\perp\right\}$ and $\mathrm{Blt}_{1}^{\prime}, \ldots, \mathrm{Blt}_{N}^{\prime}$ are such that for all $j \in[N], \mathrm{Blt}_{j}^{\prime}=\mathrm{Blt}_{j}$ if $j \notin S$ and $\mathrm{Blt}_{j}^{\prime}=\perp$ otherwise, it holds that:

If $(y, \gamma) \triangleq$ EvalTally $\left(\mathrm{Pk}, \mathrm{Blt}{ }_{1}^{\prime}, \ldots, \mathrm{Blt}_{N}^{\prime}\right)$ then VerifyTally $\left(\mathrm{Pk}, \mathrm{Blt}_{1}^{\prime}, \ldots, \mathrm{Blt}_{N}^{\prime}\right.$, $y, \gamma)=\mathrm{OK}$.

- Weak verifiability. We require the following conditions (1) and (2) to hold.

1. For all $\mathrm{Pk} \in\{0,1\}^{\star}, \mathrm{Blt}_{1}, \ldots, \mathrm{Blt}_{N} \in\{0,1\}^{\star} \cup\{\perp\}$, there exist $m_{1}, \ldots, m_{N} \in$ $\mathcal{M} \cup\{\perp\}$ such that for all $y \neq \perp$ and $\gamma$ in $\{0,1\}^{\star}$, if $S \triangleq\left\{j \mid \mathrm{Blt}_{j} \neq\right.$ $\perp \wedge$ VerifyBallot $\left.\left(\mathrm{Pk}, j, \mathrm{Blt} \mathrm{t}_{j}\right)=\perp\right\}$ and $\mathrm{Blt}_{1}^{\prime}, \ldots, \mathrm{Blt}_{N}^{\prime}$ are such that for all $j \in[N], \mathrm{Blt}_{j}^{\prime}=\mathrm{Blt}_{j}$ if $j \notin S$ and $\mathrm{Blt}_{j}^{\prime}=\perp$ otherwise, it holds that:

if VerifyTally $\left(\mathrm{Pk}, \mathrm{Blt}_{1}^{\prime}, \ldots, \mathrm{Blt}_{N}^{\prime}, y, \gamma\right)=\mathrm{OK}$ then $y=F\left(m_{1}, \ldots, m_{N}\right)$.

2. For all $\mathrm{Pk} \in\{0,1\}^{\lambda}$, all $k \in[N], i_{1}, \ldots, i_{k} \in[N]$, all $m_{i_{1}}, \ldots, m_{i_{k}} \in$ $\mathcal{M} \cup\{\perp\}$, all $\mathrm{Blt}_{1}, \ldots$, Blt $_{N} \in\{0,1\}^{\star} \cup\{\perp\}$ such that for all $j \in[k]$, $\mathrm{Blt} \mathrm{t}_{j} \in \mathrm{Cast}\left(\mathrm{Pk}, m_{i_{j}}\right)$ and VerifyBallot$\left(\mathrm{Pk}, \mathrm{Blt} \mathrm{t}_{j}\right)=\mathrm{OK}$, if $S \triangleq\left\{j \mid \mathrm{Blt} \mathrm{t}_{j} \neq\right.$ $\perp \wedge$ VerifyBallot $\left.\left(\mathrm{Pk}, j, \mathrm{Blt} \mathrm{t}_{j}\right)=\perp\right\}$ and $\mathrm{Blt}_{1}^{\prime}, \ldots, \mathrm{Blt}_{N}^{\prime}$ are such that for all $j \in[N], \mathrm{Blt}_{j}^{\prime}=\mathrm{Blt}_{j}$ if $j \notin S$ and $\mathrm{Blt}_{j}^{\prime}=\perp$ otherwise, it holds that:

if there exist $y \in\{0,1\}^{\star}$ and $\gamma \in\{0,1\}^{\star}$ such that VerifyTally(Pk, Blt ${ }_{1}^{\prime}$, $\left.\ldots, \mathrm{Blt}_{N}^{\prime}, y, \gamma\right)=\mathrm{OK}$, then $y$ is compatible with $m_{i_{1}}, \ldots, m_{i_{k}}$ at indices $i_{1}, \ldots, i_{k}$.

- Verifiability. We require the following conditions (1) and (2) to hold.

1. For all $\mathrm{Pk} \in\{0,1\}^{\star}, \mathrm{Blt}_{1}, \ldots, \mathrm{Blt}_{N} \in\{0,1\}^{\star} \cup\{\perp\}$, there exist $m_{1}, \ldots$, $m_{N} \in \mathcal{M} \cup\{\perp\}$ such that for all $y \in\{0,1\}^{\star} \cup\{\perp\}$ and $\gamma$ in $\{0,1\}^{\star}$, if $S \triangleq\left\{j \mid \mathrm{Blt}_{j} \neq \perp \wedge\right.$ VerifyBallot $\left.\left(\mathrm{Pk}, j, \mathrm{Blt}_{j}\right)=\perp\right\}$ and $\mathrm{Blt}_{1}^{\prime}, \ldots, \mathrm{Blt}_{N}^{\prime}$ are such that for all $j \in[N], \mathrm{Blt}_{j}^{\prime}=\mathrm{Blt}_{j}$ if $j \notin S$ and $\mathrm{Blt}_{j}^{\prime}=\perp$ otherwise, it holds that:

if VerifyTally $\left(\mathrm{Pk}, \mathrm{Blt}_{1}^{\prime}, \ldots, \mathrm{Blt}_{N}^{\prime}, y, \gamma\right)=\mathrm{OK}$ then $y=F\left(m_{1}, \ldots, m_{N}\right)$.

2. For all $\mathrm{Pk} \in\{0,1\}^{\lambda}$, all $k \in[N], i_{1}, \ldots, i_{k} \in[N]$, all $m_{i_{1}}, \ldots, m_{i_{k}} \in$ $\mathcal{M} \cup\{\perp\}$, all $\mathrm{Blt}_{1}, \ldots$, Blt $_{N} \in\{0,1\}^{\star} \cup\{\perp\}$ such that for all $j \in[k]$, $\mathrm{Blt}_{j} \in \operatorname{Cast}\left(\mathrm{Pk}, m_{i_{j}}\right)$ and VerifyBallot $\left(\mathrm{Pk}, \mathrm{Blt}{ }_{j}\right)=\mathrm{OK}$, if $S \triangleq\left\{j \mid \mathrm{Blt}_{j} \neq\right.$

\footnotetext{
${ }^{8}$ In the following definition, we need Abst to differentiate the case of a voter who did not cast a vote at all (Abst) from the case of a voter who casts $\perp$ as her own vote but wishes to preserve the anonymity of her choice. However, in both cases, correctness guarantees that the result of the election equals the output of the tally function, and the input to the tally function is $\perp$ both when a voter casts $\perp$ and when a voter does not cast any vote.
} 
$\perp \wedge$ VerifyBallot $\left.\left(\mathrm{Pk}, j, \mathrm{Blt} \mathrm{t}_{j}\right)=\perp\right\}$ and $\mathrm{Blt}_{1}^{\prime}, \ldots, \mathrm{Blt}_{N}^{\prime}$ are such that for all $j \in[N], \mathrm{Blt}_{j}^{\prime}=\mathrm{Blt}_{j}$ if $j \notin S$ and $\mathrm{Blt}_{j}^{\prime}=\perp$ otherwise, it holds that:

if there exist $y \in\{0,1\}^{\star} \cup\{\perp\}$ and $\gamma \in\{0,1\}^{\star}$ such that VerifyTally(Pk, $\left.\mathrm{Blt}_{1}^{\prime}, \ldots, \mathrm{Blt}_{N}^{\prime}, y, \gamma\right)=\mathrm{OK}$, then $y$ is compatible with $m_{i_{1}}, \ldots, m_{i_{k}}$ at indices $i_{1}, \ldots, i_{k}$.

Note that the difference between condition (2) of verifiability and condition (2) of weak verifiability lies in the fact that, in the latter, $y$ cannot equal $\perp$, whereas, in the former, the condition has to hold even for $y=\perp$. In this work, we use the terms verifiability and full verifiability interchangeably to differentiate them from weak verifiability.

Privacy We define privacy in the style of indistinguishability-based security. Privacy for a $(N, \mathcal{M}, \Sigma, F)$-eVote

$$
\text { EVOTE } \triangleq(\text { Setup, Cast, VerifyBallot, EvalTally, VerifyTally) }
$$

is formalized by means of the game $\operatorname{Priv}_{\mathcal{A}}^{N, \mathcal{M}, \Sigma, F \text {,EVOTE }}$ between a stateful adversary $\mathcal{A}$ and a challenger $\mathcal{C}$. We describe the game in Fig. 3 .

The advantage of adversary $\mathcal{A}$ in the above game is defined as

$$
\operatorname{Adv}_{\mathcal{A}}^{\text {EVOTE,Priv }}\left(1^{\lambda}\right) \triangleq\left|\operatorname{Prob}\left[\operatorname{Priv}_{\mathcal{A}}^{N, \mathcal{M}, \Sigma, F, \text { EVOTE }}\left(1^{\lambda}\right)=1\right]-1 / 2\right|
$$

Definition 4 An EVOTE for parameters $(N, \mathcal{M}, \Sigma, F)$ is private or IND-Secure if the advantage of all PPT adversaries $\mathcal{A}$ is at most negligible in $\lambda$ in the above game.

Definition 5 An EVOTE for parameters $(N, \mathcal{M}, \Sigma, F)$ is weakly private or wINDSecure if the advantage of all PPT adversaries $\mathcal{A}$ is at most negligible in $\lambda$ in a game WeakPriv ${ }_{\mathcal{A}}^{N, \mathcal{M}, \Sigma, F, \operatorname{EVOTE}}\left(1^{\lambda}\right)$ identical to the one above except that $\mathcal{A}$ is required to output an empty set $S$, i.e., $\mathcal{A}$ cannot submit dishonest ballots.

Remark 1 We make some remarks on the previous definitions.

- Our definitions suppose that algorithm VerifyBallot is run on input each ballot before running algorithm VerifyTally. The ballots that are input to VerifyTally are replaced by $\perp$ if they were not accepted by VerifyBallot. Another possibility would be to let VerifyTally do this task itself.

- We require that VerifyBallot and VerifyTally be deterministic algorithms. Alternatively, they can be defined as PPT, but then definitions of weak verifiability and verifiability would have to be changed accordingly to hold with probability 1 over the random coins of the algorithms.

- Our definition is parameterized by the number of voters $N$. It is possible to define a more restricted eVote that may possibly be "unbounded". Note that our definition is more general and, for instance, takes into account e-voting schemes in which the public key is of size proportional to the number of voters. 


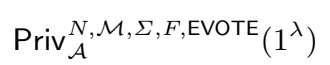

- Setup phase. $\mathcal{C}$ generates $(\mathrm{Pk}, \mathrm{Sk}) \leftarrow \operatorname{Setup}\left(1^{\lambda}\right)$, chooses a random bit $b \leftarrow$ $\{0,1\}$ and runs $\mathcal{A}$ on input Pk.

- Query phase. $\mathcal{A}$ outputs two tuples $M_{0} \triangleq\left(m_{0,1}, \ldots, m_{0, N}\right)$ and $M_{1} \triangleq$ $\left(m_{1,1}, \ldots, m_{1, N}\right)$, and a set $S \subset[N]$. (The set $S$ contains the indices of the strings in the tuples that are possibly dishonest ballots. The strings in the tuples whose indices are not in $S$ are supposed to be votes to be given as input to the Cast algorithm.)

- Challenge phase. The challenger does the following. For all $j \in[N]$, if $j \in$ $S$, then set $\mathrm{Blt}_{j} \triangleq m_{b, j}$, else set $\mathrm{Blt}_{j} \leftarrow \operatorname{Cast}\left(\mathrm{Pk}, j, m_{b, j}\right)$. For all $j \in S$, if VerifyBallot $\left(\mathrm{Pk}, j, \mathrm{Blt}_{j}\right)=\perp$, set Blt ${ }_{j} \triangleq \perp$. Compute $(y, \gamma) \leftarrow$ EvalTally $(\mathrm{Pk}, \mathrm{Sk}$, $\left.\mathrm{Blt}_{1}, \ldots, \mathrm{Blt}_{N}\right)$ and return $\left(\mathrm{Blt}_{1}, \ldots, \mathrm{Blt}_{N}, y, \gamma\right)$ to the adversary.

- Output. At some point the adversary outputs its guess $b^{\prime}$.

- Winning condition. The adversary wins the game if all the following conditions hold:

1. $b^{\prime}=b$.

2. For all $j \in S, m_{0, j}=m_{1, j}$. (That is, if the adversary submits a dishonest ballot, it has to be the same in both tuples.)

3. For all $d_{1}, \ldots, d_{N} \in \mathcal{M} \cup\{\perp\}$, for all $j \in[N]$, let $m_{0, j}^{\prime} \triangleq m_{1, j}^{\prime} \triangleq d_{j}$ if $j \in S$, and for all $b \in\{0,1\}$ let $m_{b, j}^{\prime} \triangleq m_{b, j}$ if $m_{b, j} \in \mathcal{M}$ and $m_{b, j}^{\prime} \triangleq \perp$ if $m_{b, j} \notin \mathcal{M}$. Then, $F\left(m_{0,1}^{\prime}, \ldots, m_{0, N}^{\prime}\right)=F\left(m_{1,1}^{\prime}, \ldots, m_{1, N}^{\prime}\right)$.

(That is, the tally function outputs the same result on input both tuples, even if the ballots corresponding to indices in $S$ are replaced by arbitrary messages in $\mathcal{M} \cup\{\perp\}$.)

Fig. 3. Definition of privacy 
- Both condition (2) of verifiability and condition (2) of weak verifiability lie in some sense between correctness and verifiability as they state a requirement about honest voters.

- In our weakly verifiable construction in Section 4, algorithm VerifyBallot could be completely discarded because it accepts any ballot. Both for the sake of generality (there could exist some weakly verifiable eVote that makes a non-trivial use of VerifyBallot) and to avoid overburdening the presentation, we use the same syntax for weakly verifiable eVotes and for verifiable eVotes.

- For the necessity of condition (2) of correctness, we refer the reader to the discussion in Section 1.4.

- As shown in $\left[\mathrm{BCG}^{+} 15\right]$, the definition of "Benaloh" (recall that we restate it using modern terminology) is subject to attacks when instantiated with specific tally functions like the majority. Nonetheless, ours is strengthened to withstand such attacks. This is done by adding the 3-rd winning condition.

\section{Building Blocks}

Our constructions use perfectly binding commitment schemes, (one-message) non-interactive witness-indistinguishable proof systems with perfect soundness for NP GOS06] (see also [FLS90DN00|DN00|BOV03BP15) and IND-CPA public key encryption with perfect correctness and unique secret key. In this section, we recall the definitions of those primitives.

Definition 6 [IND-CPA secure PKE with perfect correctness and unique secret key] An IND-CPA (or semantically) secure Public Key Encryption (PKE) scheme consists of three PPT algorithms (Setup, Encrypt, Decrypt) defined as follows.

- Setup $\left(1^{\lambda}\right)$ : On input $1^{\lambda}$, it outputs public key Pk and decryption key Sk.

- Encrypt $(m, \mathrm{Pk})$ : On input message $m$ and the public key, it outputs ciphertext Ct.

- Decrypt(Ct, Sk): On input ciphertext $\mathrm{Ct}$ and the decryption key, it outputs $m$.

The PKE scheme is said to be IND-CPA (or semantically) secure if for any PPT adversary $\mathcal{A}$, there exists a negligible function $\nu(\cdot)$ such that the following is satisfied for any two messages $m_{0}, m_{1}$ and for $b \in\{0,1\}$ :

$$
\left|\operatorname{Pr}\left[\mathcal{A}\left(1^{\lambda}, \operatorname{Encrypt}\left(m_{0}, \mathrm{Pk}\right)\right)=b\right]-\operatorname{Pr}\left[\mathcal{A}\left(1^{\lambda}, \operatorname{Encrypt}\left(m_{1}, \mathrm{Pk}\right)\right)=b\right]\right| \leq \nu(\lambda) .
$$

Perfect correctness requires that, for all pairs $(\mathrm{Pk}, \mathrm{Sk}) \in$ Setup, for all messages $m$ in the message space and all ciphertexts $C t$ output by Encrypt $(\mathrm{Pk}, m)$, $\operatorname{Decrypt}(\mathrm{Ct}, \mathrm{Sk})=m$ must hold. Unique secret key requires that, for all $\mathrm{Pk}$, there exists at most one Sk such that $(\mathrm{Pk}, \mathrm{Sk}) \in \operatorname{Setup}\left(1^{\lambda}\right)$.

The Decision Linear Encryption scheme BBS04 fulfills those properties. It is secure under the Decision Linear Assumption [BBS04. We recall them next. 
Bilinear Groups. We assume the existence of a PPT algorithm $\mathcal{G}\left(1^{\lambda}\right)$, the bilinear group generator, that outputs a pairing group setup $\left(p, \mathbb{G}, \mathbb{G}_{t}, \mathbf{e}, g\right)$, where $\mathbb{G}$ and $\mathbb{G}_{t}$ are multiplicative groups of prime order $p$ and $\mathbf{e}: \mathbb{G} \times \mathbb{G} \rightarrow \mathbb{G}_{t}$ is a bilinear map satisfying the following three properties: (1) bilinearity, i.e., $\mathbf{e}\left(g^{x}, g^{y}\right)=$ $e(g, g)^{x y} ;(2)$ non-degeneracy, i.e., for all generators $g \in \mathbb{G}, \mathbf{e}(g, g)$ generates $\mathbb{G}_{t}$; (3) efficiency, i.e., e can be computed in polynomial time.

Assumption 1 (Decision Linear Assumption for $\mathcal{G}$. [BBS04]) Let the tuple $\left(p, \mathbb{G}, \mathbb{G}_{t}, \mathbf{e}, g\right)$ be a pairing group setup output by $\mathcal{G}$ as defined above, and let $g_{1}, g_{2}$ and $g_{3}$ be generators of $\mathbb{G}$. Given $\left(g_{1}, g_{2}, g_{3}, g_{1}^{a}, g_{2}^{b}, g_{3}^{c}\right)$, where $a$ and $b$ are picked randomly from $\mathbb{Z}_{p}$, the Decision Linear (DLIN) assumption is to decide whether $c=a+b \bmod p$. Precisely, the advantage of an adversary $\mathcal{A}$ in solving the Decision Linear assumption is given by:

$$
\begin{aligned}
& \mid \operatorname{Pr}\left[\mathcal{A}\left(\mathbb{G}, p, g_{1}, g_{2}, g_{3}, g_{1}^{a}, g_{2}^{b}, g_{3}^{a+b}\right)=1 \mid\left(p, \mathbb{G}, \mathbb{G}_{t}, \mathbf{e}, g\right) \leftarrow \mathcal{G}\left(1^{\lambda}\right) ;\right. \\
& \left.\left(g_{1}, g_{2}, g_{3}\right) \leftarrow \mathbb{G} ;(a, b) \leftarrow \mathbb{Z}_{p}\right]- \\
& \operatorname{Pr}\left[\mathcal{A}\left(\mathbb{G}, p, g_{1}, g_{2}, g_{3}, g_{1}^{a}, g_{2}^{b}, g_{3}^{c}\right)=1 \mid\left(p, \mathbb{G}, \mathbb{G}_{t}, \mathbf{e}, g\right) \leftarrow \mathcal{G}\left(1^{\lambda}\right) ;\right. \\
& \left.\left(g_{1}, g_{2}, g_{3}\right) \leftarrow \mathbb{G} ;(a, b, c) \leftarrow \mathbb{Z}_{p}\right] \mid
\end{aligned}
$$

The Decision Linear assumption states that the advantage of $\mathcal{A}$ is negligible in $\lambda$. Boneh et al. BBS04 provide a bilinear group generator $\mathcal{G}$ for which such assumption is conjectured to hold.

Decision Linear Encryption Scheme. Consider the following PKE scheme described by a setup algorithm Setup, an encryption algorithm Encrypt and a decryption algorithm Decrypt.

$\operatorname{Setup}\left(1^{\lambda}\right):$ pick $\left(p, \mathbb{G}, \mathbb{G}_{t}, \mathbf{e}, g\right) \leftarrow \mathcal{G}\left(1^{\lambda}\right)$, pick randomly $g_{3} \leftarrow \mathbb{G}$ and $(x, y) \leftarrow \mathbb{Z}_{p}$. Compute $g_{1}=g_{3}^{1 / x}$ and $g_{2}=g_{3}^{1 / y}$. Output the public key Pk $=\left(\mathbb{G}, p, g_{1}, g_{2}\right.$, $\left.g_{3}\right)$ and the secret key $\mathrm{Sk}=(\mathrm{Pk}, x, y)$.

Encrypt $(\mathrm{Pk}, m)$ : on input a public key $\mathrm{Pk}$ and a message $m \in \mathbb{G}$, pick random $(a, b) \leftarrow \mathbb{Z}_{p}$. Output a ciphertext $\mathrm{Ct}=\left(g_{1}^{a}, g_{2}^{b}, m \cdot g_{3}^{a+b}\right)$.

$\operatorname{Decrypt}(\mathrm{Sk}, \mathrm{Ct})$ : on input a secret key $\mathrm{Sk}$ and a ciphertext $\mathrm{Ct}=\left(c_{1}, c_{2}, c_{3}\right)$, output $m=c_{3} /\left(c_{1}^{x} c_{2}^{y}\right)$.

This scheme fulfills the IND-CPA property under the Decision Linear assumption (see BBS04 for details) and it is easy to verify that it fulfills the unique secret key property.

Definition 7 [(Perfectly binding) Commitment Schemes] A commitment scheme Com is a PPT algorithm that takes as input a string $x$ and randomness $r \in$ $\{0,1\}^{k}$ and outputs com $\leftarrow \operatorname{Com}(x ; r)$. A perfectly binding commitment scheme must satisfy the following properties:

- Perfectly Binding: This property states that two different strings cannot have the same commitment. More formally, $\forall x_{1} \neq x_{2}$ and $r_{1}, r_{2}, \operatorname{Com}\left(x_{1} ; r_{1}\right) \neq$ $\operatorname{Com}\left(x_{2} ; r_{2}\right)$. 
- Computational Hiding: For all strings $x_{0}$ and $x_{1}$ (of the same length), for all PPT adversaries $\mathcal{A}$ there exists a negligible function $\nu(\cdot)$ such that: $\left.\mid \operatorname{Pr}_{r \in\{0,1\}^{k}}\left[\mathcal{A}\left(\operatorname{Com}\left(x_{0} ; r\right)\right)=1\right]-\operatorname{Pr}_{r \in\{0,1\}^{k}}\left[\mathcal{A}\left(\operatorname{Com}\left(x_{1} ; r\right)\right)=1\right)\right] \mid \leq \nu(k)$.

NIWI proof systems. Next, we define (one-message) non-interactive witness indistinguishability (NIWI) proof systems GOS06]. Groth et al. GOS06 construct such NIWIs for all languages in NP, and in particular for CircuitSat.

Definition 8 [Non-interactive Proof System] A non-interactive proof system for a language $L$ with a PPT relation $R$ is a tuple of algorithms (Prove, Verify). Prove receives as input a statement $x$ and a witness $w$ and outputs a proof $\pi$. Verify receives as input a statement $x$ and a proof $\pi$ and outputs a symbol in $\{\mathrm{OK}, \perp\}$. The following properties must hold:

- Perfect Completeness: For every $(x, w) \in R$, it holds that $\operatorname{Pr}[\operatorname{Verify}(x, \operatorname{Prove}(x, w))=\mathrm{OK}]=1$, where the probability is taken over the coins of Prove and Verify.

- Perfect Soundness: For every adversary $\mathcal{A}$, it holds that:

$$
\operatorname{Pr}\left[\begin{array}{l}
\operatorname{Verify}(x, \pi)=\mathrm{OK} \wedge x \notin L: \\
(x, \pi) \leftarrow \mathcal{A}\left(1^{k}\right)
\end{array}\right]=0 .
$$

Definition 9 [NIWI] A non-interactive proof system NIWI = (Prove, Verify) for a language $L$ with a PPT relation $R$ is witness-indistinguishable (WI, in short) if for any triplet $\left(x, w_{0}, w_{1}\right)$ such that $\left(x, w_{0}\right) \in R$ and $\left(x, w_{1}\right) \in R$, the distributions $\left\{\operatorname{Prove}\left(x, w_{0}\right)\right\}$ and $\left\{\operatorname{Prove}\left(x, w_{1}\right)\right\}$ are computationally indistinguishable.

\section{Our Weakly Verifiable eVote}

In this section, we present our weakly verifiable eVote EVOTE. This eVote fulfills the wIND-Security and weak verifiability properties.

Definition 10 [EVOTE] Let NIWI ${ }^{\text {dec }}=\left(\right.$ Prove $^{\text {dec }}$, Verify $\left.^{\text {dec }}\right)$ be a NIWI proof system for the relation $\mathrm{R}^{\text {dec }}$, which we specify later. Let $\mathcal{E}=(\mathcal{E}$.Setup, $\mathcal{E}$.Encrypt, $\mathcal{E}$.Decrypt) be a PKE scheme with perfect correctness and unique secret key (see Def. 6).

We define as follows an $(N, \mathcal{M}, \Sigma, F)$-eVote

$$
\text { EVOTE }^{N, \mathcal{M}, \Sigma, F, \mathcal{E}, \text { NIII }^{\text {dec }}}=(\text { Setup, Cast, VerifyBallot, EvalTally, VerifyTally })
$$

- Setup $\left(1^{\lambda}\right)$ : on input the security parameter in unary, do the following.

1. For all $l \in[3] \operatorname{run}\left(\mathcal{E} . \mathrm{Pk}_{l}, \mathcal{E} . \mathrm{Sk}_{l}\right)=\mathcal{E}$.Setup $\left(1^{\lambda} ; s_{l}\right)$ with randomness $s_{l}$.

2. Output $\mathrm{Pk} \triangleq\left(\mathcal{E} . \mathrm{Pk}_{1}, \ldots, \mathcal{E} . \mathrm{Pk}_{3}\right)$ and $\mathrm{Sk} \triangleq\left(\mathcal{E} . \mathrm{Sk}_{1}, \mathcal{E} . \mathrm{Sk}_{2}, s_{1}, s_{2}\right) 9$

${ }^{9}$ Actually, as the randomness for the setup of our PKE scheme uniquely determines the secret key, it would be sufficient to just include the $s_{l}$ 's in Sk. 
- Cast $(\mathrm{Pk}, j, v)$ : on input the public key $\mathrm{Pk}$, the voter index $j \in[N]$, and a vote $v$, do the following.

1. For all $l \in[3]$, compute $\mathrm{Ct}_{j, l} \leftarrow \mathcal{E}$. $\operatorname{Encrypt}\left(\mathcal{E}\right.$. $\left.\mathrm{Pk}_{l}, v\right)$.

2. Output $\mathrm{Blt}_{j} \triangleq\left(\mathrm{Ct}_{j, 1}, \ldots, \mathrm{Ct}_{j, 3}\right)$.

- VerifyBallot(Pk, $j$, Blt): on input the public key $\mathrm{Pk}$, the voter index $j \in[N]$, and a ballot Blt, output OK (i.e., accept any ballot, even invalid ones).

- EvalTally $\left(\mathrm{Pk}, \mathrm{Sk}, \mathrm{Blt} \mathrm{t}_{1}, \ldots, \mathrm{Blt}_{N}\right)$ : on input the public key $\mathrm{Pk}$, the secret key Sk, and a tuple of $N$ strings $\left(\mathrm{Blt}_{1}, \ldots, \mathrm{Blt}_{N}\right)$ that consists of either ballots cast by a voter or the special symbol $\perp$, do the following.

1. For all $j \in[N], l \in[2]$,

$$
m_{j}^{l}= \begin{cases}\perp & \text { if } \mathrm{Blt}_{j}=\perp, \\ \perp & \text { if Blt } \neq \perp \wedge \mathcal{E} \text {.Decrypt }\left(\mathrm{Ct}_{j, l}, \mathcal{E} . \mathrm{Sk}_{l}\right) \notin \mathcal{M}, \\ \mathcal{E} \text {.Decrypt }\left(\mathrm{Ct}_{j, l}, \mathcal{E} . \mathrm{Sk}_{l}\right) & \text { otherwise. }\end{cases}
$$

2. For all $l \in[2]$, compute $y_{l}=F\left(m_{1, l}, \ldots, m_{N, l}\right)$.

3 . If $y_{1}=y_{2}$, then set $y=y_{1}$, else set $y=\perp$.

4. Consider the following relation $\mathrm{R}^{\mathrm{dec}}$ in Fig. 4. Henceforth, if the indices $\left(i_{1}, i_{2}\right)$ in the witness of the relation $\mathrm{R}^{\text {dec }}$ fulfill $i_{1}=1$ and $i_{2}=2$ (resp. $i_{1} \neq 1$ or $i_{2} \neq 2$ ), the statement or the proof is in real mode (resp. trapdoor mode). Set the statement

$$
x \triangleq\left(\mathrm{Blt}_{1}, \ldots, \mathrm{Blt}_{N}, \mathcal{E} \cdot \mathrm{Pk}_{1}, \ldots, \mathcal{E} \cdot \mathrm{Pk}_{3}, y\right)
$$

and the witness

$$
w \triangleq\left(\mathcal{E} . \mathrm{Sk}_{1}, \mathcal{E} . \mathrm{Sk}_{2}, s_{1}, s_{2}, i_{1} \triangleq 1, i_{2} \triangleq 2\right)
$$

and compute a proof $\gamma \leftarrow \operatorname{Prove}^{\mathrm{dec}}(x, w)$.

5. Output $(y, \gamma)$.

- VerifyTally $\left(\mathrm{Pk}, \mathrm{Blt}_{1}, \ldots, \mathrm{Blt}_{N}, y, \gamma\right)$ : on input the public key Pk, a tuple of $N$ strings that can be either ballots cast by a voter or the special symbol $\perp$, a tally $y$ and a proof $\gamma$, if $\gamma=\perp$ output $\perp$, else set

$$
x \triangleq\left(\mathrm{Blt}_{1}, \ldots, \mathrm{Blt}_{N}, \mathcal{E} \cdot \mathrm{Pk}_{1}, \ldots, \mathcal{E} \cdot \mathrm{Pk}_{3}, y\right)
$$

output Verify $^{\text {dec }}(x, \gamma)$.

Henceforth, for simplicity we omit the parameters of the scheme and we write just EVOTE.

\subsection{Correctness and Weak Verifiability of the Construction}

Correctness. The (perfect) correctness of EVOTE follows from the perfect correctness of $\mathcal{E}$ and the perfect completeness of NIWI ${ }^{\text {dec }}$. 
Relation $\mathrm{R}^{\mathrm{dec}}(x, w)$ :

Instance: $x \triangleq\left(\mathrm{Blt}_{1}, \ldots, \mathrm{Blt}_{N}, \mathcal{E} \cdot \mathrm{Pk}_{1}, \ldots, \mathcal{E} \cdot \mathrm{Pk}_{3}, y\right)$. (Recall that a ballot is set to $\perp$ if the corresponding voter did not cast her vote.)

Witness: $w \triangleq\left(\mathcal{E} . \mathrm{Sk}_{1}^{\prime}, \mathcal{E} . \mathrm{Sk}_{2}^{\prime}, s_{1}, s_{2}, i_{1}, i_{2}\right)$, where the $s_{l}$ 's are the randomness used to generate the secret keys and public keys (which are known to the authority who set up the system).

$\mathrm{R}^{\mathrm{dec}}(x, w)=1$ if and only if the following condition holds.

2 of the secret keys corresponding to indices $\mathcal{E} . \mathrm{Pk}_{i_{1}}, \mathcal{E} . \mathrm{Pk}_{i_{2}}$ are constructed using honestly generated public and secret key pairs and are equal to $\mathcal{E} . \mathrm{Sk}_{1}^{\prime}, \mathcal{E} . \mathrm{Sk}_{2}^{\prime}$; and either $y=\perp$ or for all $l \in[2], y=F\left(m_{1}^{l}, \ldots, m_{N}^{l}\right)$ and for all $j \in[N]$, if Blt $_{j} \neq \perp$ then for $l \in[2], \mathcal{E} . \mathrm{Sk}_{i_{l}}$ decrypts ciphertext $\mathrm{Ct}_{j, i_{l}}$ in Blt ${ }_{j}$ to $m_{j}^{i_{l}} \in \mathcal{M}$; and for all $l \in[2], m_{j}^{l}=\perp$ if either $\mathrm{Blt}_{j}=\perp$ or $\mathcal{E}$.Sk $\mathrm{Sk}_{i_{l}}$ decrypts $\mathrm{Ct}_{j, i_{l}}$ to a string $\notin \mathcal{M}$.

Precisely, $\mathrm{R}^{\operatorname{dec}}(x, w)=1$ if and only if the following conditions hold. In the following, items (a) and (c) are not actually conditions that have to be checked

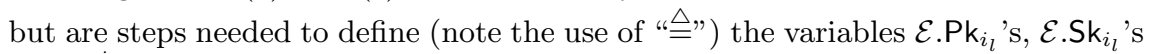
and $m_{j}^{i_{l}}$ 's that are used in the checks (b) and (d).

(a) For all $l \in[2],\left(\mathcal{E} . \mathrm{Pk}_{i_{l}}, \mathcal{E} \cdot \mathrm{Sk}_{i_{l}}\right) \triangleq \mathcal{E} \cdot \operatorname{Setup}\left(1^{\lambda} ; s_{l}\right)$.

(b) For all $l \in[2], \mathcal{E} . \mathrm{Sk}_{l}^{\prime}=\mathcal{E} . \mathrm{Sk}_{i_{l}}$.

(c) For all $j \in[N], l \in[2]$,

$$
m_{j}^{i_{l}} \triangleq \begin{cases}\perp & \text { if } \mathrm{Blt}_{j}=\perp \\ \perp & \text { if Blt } \mathrm{Bl}_{j} \neq \perp \wedge \mathcal{E} \text {.Decrypt }\left(\mathrm{Ct}_{j, i_{l}}, \mathcal{E} . \mathrm{Sk}_{i_{l}}\right) \notin \mathcal{M} \\ \mathcal{E} . \operatorname{Decrypt}\left(\mathrm{Ct}_{j, i_{l}}, \mathcal{E} . \mathrm{Sk}_{i_{l}}\right) & \text { otherwise. }\end{cases}
$$

(d) $(y=\perp) \vee\left(\right.$ for all $\left.l \in[2], y=F\left(m_{1}^{i_{l}}, \ldots, m_{N}^{i_{l}}\right)\right)$.

(Note that $\mathcal{E} . \mathrm{Sk}_{1}^{\prime}$ and $\mathcal{E} . \mathrm{Sk}_{2}^{\prime}$ do not necessarily have to correspond to the first two secret keys.)

Fig. 4. Relation $\mathrm{R}^{\mathrm{dec}}$ 


\section{Weak verifiability.}

Theorem 1 For all $N>0$, all sets $\mathcal{M}, \Sigma \subset\{0,1\}^{\star}$, and all tally functions $F:(\mathcal{M} \cup\{\perp\})^{N} \rightarrow \Sigma \cup\{\perp\}$, if $\mathcal{E}$ is a perfectly correct PKE with unique secret key (cf. Def. 6) and NIWI ${ }^{\text {dec }}$ is a (one-message) NIWI (cf. Def. 9) for the relation $\mathrm{R}^{\text {dec }}$, then EVOTE ${ }^{N, \mathcal{M}, \Sigma, F, \mathcal{E}, \text { NIWIdec }^{\text {dec }}}$ satisfies the weak verifiability property (cf. Def. 3).

Proof. First, we prove that condition (1) of verifiability is satisfied. Since algorithm VerifyBallot accepts any ballot, even invalid ones, we have to prove that for all $\mathrm{Pk} \in\{0,1\}^{\star}$, all $\mathrm{Blt}_{1}, \ldots, \mathrm{Blt}_{N} \in\{0,1\}^{\star} \cup\{\perp\}$, there exist $m_{1}, \ldots, m_{N} \in$ $\mathcal{M} \cup\{\perp\}$ such that for all $y \neq \perp$ and all $\gamma$ in $\{0,1\}^{\star}$, if VerifyTally $\left(\mathrm{Pk}, \mathrm{Blt}_{1}, \ldots\right.$, $\left.\mathrm{Blt}_{N}, y, \gamma\right)=1$ then $y=F\left(m_{1}, \ldots, m_{N}\right)$.

Henceforth, w.l.o.g, we let $\mathrm{Pk}$ and $\mathrm{Blt}_{1}, \ldots, \mathrm{Blt}_{N}$ be arbitrary strings. First, we prove the following claim.

Claim 1 Given $\mathrm{Pk}$ and $\left(\mathrm{Blt}_{1}, \ldots, \mathrm{Blt}_{N}\right)$, for every two pairs $\left(y_{0}, \gamma_{0}\right)$ and $\left(y_{1}, \gamma_{1}\right)$ such that $y_{0}, y_{1} \neq \perp$, if VerifyTally $\left(\mathrm{Pk}, \mathrm{Blt}_{1}, \ldots, \mathrm{Blt}_{N}, y_{0}, \gamma_{0}\right)=$ VerifyTally $(\mathrm{Pk}$, $\left.\mathrm{Blt}_{1}, \ldots, \mathrm{Blt}_{N}, y_{1}, \gamma_{1}\right)=\mathrm{OK}$ then $y_{0}=y_{1}$.

Let $y_{0}, \gamma_{0}, y_{1}, \gamma_{1}$ be arbitrary strings in $\{0,1\}^{\star} \cup\{\perp\}$ such that $y_{0}, y_{1} \neq \perp$. Suppose that VerifyTally $\left(\mathrm{Pk}, \mathrm{Blt}{ }_{1}, \ldots, \mathrm{Blt}_{N}, y_{0}, \gamma_{0}\right)=\operatorname{Verify} T a l l y\left(\mathrm{Pk}, \mathrm{Blt}{ }_{1}, \ldots, \mathrm{Blt}_{N}\right.$, $\left.y_{1}, \gamma_{1}\right)=$ OK. The perfect soundness of NIWI ${ }^{\text {dec }}$ implies that, for all $b \in\{0,1\}$, the proof $\gamma_{b}$ is computed on input some witness $\left(\mathcal{E} . \mathbf{S k}_{1}^{\prime b}, \mathcal{E} . \mathrm{Sk}_{2}^{\prime b}, s_{1}^{b}, s_{2}^{b}, i_{1}^{b}, i_{2}^{b}\right)$.

By the pigeon principle, there exists an index $i^{\star}$ such that one of the following cases holds.

1. $i^{\star}=i_{1}^{0}=i_{2}^{1}$. For all $b \in\{0,1\}$, let $\left(m_{1}^{i^{\star}, b}, \ldots, m_{N}^{i^{\star}, b}\right)$ be the messages guaranteed by condition (iii) of relation $\mathrm{R}^{\mathrm{dec}}$ for proof $\gamma_{b}$. Condition (i) for proof $\gamma_{0}\left(\right.$ resp. $\left.\gamma_{1}\right)$ implies that the secret key $\mathbf{S k}_{1}^{\prime 0}$ (resp. $\mathbf{S} \mathbf{k}_{2}^{\prime 1}$ ) is honestly computed and thus, the unique secret key property and the fact that it fulfills $\mathcal{E} . \mathrm{Pk}_{i_{1}^{0}}=\mathcal{E} . \mathrm{Pk}_{i^{\star}}\left(\right.$ resp. $\mathcal{E} . \mathrm{Pk}_{i_{2}^{1}}=\mathcal{E}$. $\left.\mathrm{Pk}_{i^{\star}}\right)$ imply that for all $j \in[N]$, $\mathcal{E}$.Decrypt $\left(\mathrm{Ct}_{j, i^{\star}}, \mathcal{E} . \mathrm{Sk}_{1}^{\prime 0}\right)=\mathcal{E}$.Decrypt $\left(\mathrm{Ct}_{j, i^{\star}}, \mathcal{E} . \mathrm{Sk}_{2}^{\prime 1}\right)$.

Furthermore, condition (ii) and (iii) for proof $\gamma_{0}$ (resp. $\gamma_{1}$ ) imply that for all $j \in[N]$, either $m_{j}^{i^{\star}, 0}=\perp$ or $m_{j}^{i^{\star}, 0}=\mathcal{E}$.Decrypt $\left(\mathrm{Ct}_{j, i^{\star}}, \mathcal{E}\right.$.Sk $\left.\mathrm{k}_{1}^{\prime}\right) \in \mathcal{M}$ (resp. either $m_{j}^{i^{\star}, 1}=\perp$ or $m_{j}^{i^{\star}, 1}=\mathcal{E}$. Decrypt $\left.\left(\mathrm{Ct}_{j, i^{\star}}, \mathcal{E} . \mathrm{Sk}_{2}^{\prime 1}\right) \in \mathcal{M}\right)$.

Hence, for all $j \in[N], m_{j}^{i^{\star}, 0}=m_{j}^{i^{\star}, 1} \in \mathcal{M} \cup\{\perp\}$. Now, condition (iv) for proof $\gamma_{0}$ (resp. $\left.\gamma_{1}\right)$ implies that either $y_{0}=F\left(m_{1}^{i_{1}^{0}, 0}, \ldots, m_{N}^{i_{1}^{0}, 0}\right)$ or $y_{0}=\perp$ (resp. either $y_{1}=F\left(m_{1}^{i_{2}^{1}, 1}, \ldots, m_{N}^{i_{2}^{1}, 1}\right)$ or $y_{1}=\perp$ ) and, as by hypothesis $y_{0}, y_{1} \neq \perp$, it holds that $y_{0}=y_{1}$. (Here, the "weakness" of EVOTE arises, i.e., it cannot be proven (fully) verifiable because it could occur that, for example, $y_{0} \neq y_{1}, y_{0}=\perp$.)

2. $i^{\star}=i_{2}^{0}=i_{1}^{1}$. This case is identical to the first one, except that we replace $i_{1}^{0}$ with $i_{2}^{0}$ and $i_{2}^{1}$ with $i_{1}^{1}$.

3. $i^{\star}=i_{1}^{0}=i_{1}^{1}$. This case is identical to the first one, except that we replace $i_{2}^{1}$ with $i_{1}^{1}$. 
4. $i^{\star}=i_{2}^{0}=i_{2}^{1}$. This case is identical to the first one, except that we replace $i_{1}^{0}$ with $i_{2}^{0}$.

In all cases, we have that, if VerifyTally $\left(\mathrm{Pk}, \mathrm{Blt}_{1}, \ldots, \mathrm{Blt}_{N}, y_{0}, \gamma_{0}\right)=$ VerifyTally $(\mathrm{Pk}$, $\left.\mathrm{Blt}_{1}, \ldots, \mathrm{Blt}_{N}, y_{1}, \gamma_{1}\right)=\mathrm{OK}$ then $y_{0}=y_{1}$. In conclusion, the claim is proved.

From the previous claim, it follows that there exists a unique value $y^{\star}$ such that, for all $(y, \gamma)$ such that $y \neq \perp$, if Verify Tally $\left(\mathrm{Pk}_{\mathrm{B}} \mathrm{Blt}_{1}, \ldots, \mathrm{Blt}_{N}, y, \gamma\right)=\mathrm{OK}$ then $y=y^{\star}(1)$. Moreover, it is easy to see that, for all $(y, \gamma)$, if VerifyTally $(\mathrm{Pk}$, $\left.\mathrm{Blt}_{1}, \ldots, \mathrm{Blt}_{N}, y, \gamma\right)=\mathrm{OK}$, there exist messages $m_{1}, \ldots, m_{N} \in \mathcal{M} \cup\{\perp\}$ such that $y=F\left(m_{1}, \ldots, m_{N}\right)(2)$.

Now, we have two mutually exclusive cases.

- For all $(y, \gamma)$ such that $y \neq \perp$, VerifyTally $\left(\mathrm{Pk}, \mathrm{Blt}_{1}, \ldots, \mathrm{Blt}_{N}, y, \gamma\right)=\perp$. Then, letting $m_{1}, \ldots, m_{N}$ in the statement of the theorem be arbitrary messages in $\mathcal{M} \cup\{\perp\}$, the statement is verified with respect to $\mathrm{Pk}$ and $\mathrm{Blt}_{1}, \ldots, \mathrm{Blt}_{N}$.

- There exists $\left(y^{\prime}, \gamma\right)$ such that $y^{\prime} \neq \perp$ and VerifyTally $\left(\mathrm{Pk}, \mathrm{Blt}_{1}, \ldots, \mathrm{Blt}_{N}, y^{\prime}, \gamma\right)$ $=\mathrm{OK}$. In this case, (2) implies that there exist $m_{1}^{\prime}, \ldots, m_{N}^{\prime} \in \mathcal{M} \cup\{\perp\}$ such that $y^{\prime}=F\left(m_{1}^{\prime}, \ldots, m_{N}^{\prime}\right)(3)$. Hence, (1) and (3) together imply that $y^{\star}=F\left(m_{1}^{\prime}, \ldots, m_{N}^{\prime}\right)(4)$.

Therefore, for all $(y, \gamma)$ such that $y \neq \perp$, if VerifyTally $\left(\mathrm{Pk}, \mathrm{Blt}_{1}, \mathrm{Blt}_{N}, y, \gamma\right)=$ OK then (by (1)) $y=y^{\star}=($ by $(4))=F\left(m_{1}^{\prime}, \ldots, m_{N}^{\prime}\right)$.

Then, for $m_{1} \triangleq m_{1}^{\prime}, \ldots, m_{N} \triangleq m_{N}^{\prime}$, the statement of condition (1) of weak verifiability is verified with respect to $\mathrm{Pk}$ and $\mathrm{Blt}_{1}, \ldots, \mathrm{Blt}_{N}$.

In both cases, for $m_{1} \triangleq m_{1}^{\prime}, \ldots, m_{N} \triangleq m_{N}^{\prime}$, the statement of condition (1) of weak verifiability is verified with respect to $\mathrm{Pk}$ and $\mathrm{Blt}_{1}, \ldots, \mathrm{Blt}_{N}$.

As $\mathrm{Pk}$ and $\mathrm{Blt}_{1}, \ldots, \mathrm{Blt}_{N}$ are arbitrary strings, the statement of condition (1) of weak verifiability is proven.

It is also easy to check that condition (2) of weak verifiability is satisfied. This follows straightforwardly from the perfect soundness of NIWIdec . Thanks to NIWI ${ }^{\text {dec }}$, the authority always proves that the public key of the PKE scheme is honestly generated. Therefore, by the perfect correctness of the PKE scheme, an honestly computed ballot for message $m$ for the $j$-th voter is decrypted to $m$ (because an honestly computed ballot, by definition, consists of three ciphertexts that encrypt the same message). Consequently, if the tally $y$ is different from $\perp$ (i.e., if the evaluation of the tally function is equal for all indices), then $y$ has to be compatible with $m$ at index $j$ (cf. Def. 11).

\subsection{Weak Privacy of the Construction}

Theorem 2 For all $N>0$, all sets $\mathcal{M}, \Sigma \subset\{0,1\}^{\star}$, and all tally functions $F:(\mathcal{M} \cup\{\perp\})^{N} \rightarrow \Sigma \cup\{\perp\}$, if $\mathcal{E}$ is a perfectly correct PKE scheme with unique secret key (cf. Def. 6) and NIWI ${ }^{\text {dec }}$ is a (one-message) NIWI (cf. Def. 9) for the relation $\mathrm{R}^{\text {dec }}$, then EVOTE ${ }^{N, \mathcal{M}, \Sigma, F, \mathcal{E}, \text { NIII }^{\text {dec }}}$ is wIND-Secure (cf. Def. 5 ). 
Proof. Let $\mathcal{A}$ be a PPT adversary against the wIND-Security property of EVOTE. We prove that $\operatorname{Adv}_{\mathcal{A}}^{\mathrm{EVOTE}, \text { WeakPriv }}\left(1^{\lambda}\right) \leq \nu\left(1^{\lambda}\right)$ for some negligible function $\nu(\lambda)$.

We prove that by means of a series of hybrid experiments. We refer the reader to Table 1.5 for a pictorial explanation of the experiments, which are explained in Section 1.5. In the table, for simplicity, we omit the indices $k$ for the experiments $H_{2}^{k}$ 's, $H_{4}^{k}$ 's, $H_{6}^{k}$ 's presented below. Therefore, hybrid experiment $H_{2}$ (resp. $H_{4}, H_{6}$ ) in the table corresponds to hybrid experiment $H_{2}^{N}$ (resp. $\left.H_{4}^{N}, H_{6}^{N}\right)$ below.

Hybrid $H_{1}$. Experiment $H_{1}$ is equal to the experiment WeakPriv ${ }_{\mathcal{A}}^{N, \mathcal{M}, \Sigma, F, \text { EVOTE }}$ except that the challenger sets $b \triangleq 0$.

Hybrid $H_{2}^{k}$, for $k=0, \ldots, N$. For all $k=0, \ldots, N$, experiment $H_{2}^{k}$ is identical to experiment $H_{1}$ except that, for all $j=1, \ldots, k$, the challenger computes $\mathrm{Ct}_{k, 3}$ on input $m_{1, k}$. Note that $H_{2}^{0}$ is identical to $H_{1}$.

Claim 2 For all $k=1, \ldots, N$, the advantage of $\mathcal{A}$ in distinguishing $H_{2}^{k-1}$ from $H_{2}^{k}$ is negligible.

Proof. Suppose toward a contradiction that $\mathcal{A}$ has instead non-negligible advantage $\epsilon(\lambda)$. We construct an adversary $\mathcal{B}$ that has advantage at most $\epsilon(\lambda)$ against the IND-CPA security of $\mathcal{E}$.

$\mathcal{B}$ receives from the challenger of IND-CPA a public key pk and sets $\mathrm{Pk}_{3} \triangleq \mathrm{pk}$. For $l \in[2], \mathcal{B}$ runs $\mathcal{E}$.Setup to compute $\left(\mathcal{E}\right.$. $\mathrm{Pk}_{l}, \mathcal{E}$.Sk $\left.\mathrm{Sk}_{l}\right)$ and runs $\mathcal{A}$ on input $\mathrm{Pk} \triangleq\left(\mathcal{E} . \mathrm{Pk}_{1}, \mathcal{E} \cdot \mathrm{Pk}_{2}, \mathcal{E} \cdot \mathrm{Pk}_{3}\right)$.

$\mathcal{A}$ outputs two tuples $\left(m_{0,1}, \ldots, m_{0, N}\right)$ and $\left(m_{1,1}, \ldots, m_{1, N}\right)$, and a set $S$, which is empty for the wIND-Security game. $\mathcal{B}$ returns $\left(m_{0, k}, m_{1, k}\right)$ as its pair of challenge messages to the IND-CPA challenger. The IND-CPA challenger sends $\mathcal{B}$ the challenge ciphertext $\mathrm{ct}^{\star}$.

$\mathcal{B}$ computes Blt $t_{k} \triangleq\left(\mathrm{Ct}_{k, 1}, \mathrm{Ct}_{k, 2}, \mathrm{ct}^{\star}\right)$ by encrypting $m_{0, j}$ in $\mathrm{Ct}_{k, 1}$ and $\mathrm{Ct}_{k, 2}$. $\mathcal{B}$ can compute the ballots $\mathrm{Blt}_{j}$ for all $j \in[N], j \neq k$ exactly as the challenger in both experiments would do.

$\mathcal{B}$ computes $y$ as in the previous experiment (i.e., by running EvalTally on input $\left.\left(\mathrm{Pk}, \mathrm{Blt}_{1}, \ldots, \mathrm{Blt}_{N}\right)\right)$ and uses the 2 secret keys $\left(\mathcal{E} . \mathrm{Sk}_{1}, \mathcal{E} . \mathrm{Sk}_{2}\right)$ to compute a proof $\gamma$ exactly as the challenger in both experiments would do. $\mathcal{B}$ restarts $\mathcal{A}$ on input the computed ballots along with $(y, \gamma)$ and returns the output of $\mathcal{A}$.

It is easy to see that, if $\mathrm{ct}^{\star}$ is an encryption of $m_{0, k}$, then $\mathcal{B}$ simulates experiment $H_{2}^{k-1}$, and, if $\mathrm{ct}^{\star}$ is an encryption of $m_{1, k}$, then $\mathcal{B}$ simulates experiment $H_{2}^{k}$. Therefore, $\mathcal{B}$ has probability $\epsilon(\lambda)$ of winning the IND-CPA game, which contradicts the assumption that the PKE scheme fulfills the IND-CPA property.

Hybrid $H_{3}$. Experiment $H_{3}$ is identical to experiment $H_{2}^{N}$ except that the challenger computes the proof $\gamma$ on input a witness that contains indices $(1,3)$ and secret keys $\mathrm{Sk}_{1}, \mathrm{Sk}_{3}$ (precisely, with the randomness used to compute those secret keys, but henceforth, for simplicity, we omit this detail). 
Claim 3 The advantage of $\mathcal{A}$ in distinguishing $H_{2}^{N}$ from $H_{3}$ is negligible.

Proof. This follows straightforwardly from the WI property of NIWI ${ }^{\text {dec }}$. We note that both the randomness used to compute $\mathbf{S k}_{1}, \mathrm{Sk}_{2}$ and the randomness used to compute $\mathrm{Sk}_{1}, \mathrm{Sk}_{3}$ constitute valid witnesses for the statement $\left(\mathrm{Blt}_{1}, \ldots, \mathrm{Blt} \mathrm{t}_{N}\right.$, $\left.\mathcal{E} . \mathrm{Pk}_{1}, \ldots, \mathcal{E} . \mathrm{Pk}_{3}, y\right)$.

Hybrid $H_{4}^{k}$, for $k=0, \ldots, N$. For all $k=0, \ldots, N$, experiment $H_{4}^{k}$ is identical to experiment $H_{3}$, except that, for all $j=1, \ldots, k$, the challenger computes $\mathrm{Ct}_{k, 2}$ on input $m_{1, k}$. Note that $H_{4}^{0}$ is identical to $H_{3}$.

Claim 4 For all $k=1, \ldots, N$, the advantage of $\mathcal{A}$ in distinguishing $H_{4}^{k-1}$ from $H_{4}^{k}$ is negligible.

Proof. The proof is identical to the one for Claim 2 except that the third index and the second index are swapped.

Hybrid $H_{5}$. Experiment $H_{5}$ is identical to experiment $H_{4}^{N}$ except that the challenger computes the proof $\gamma$ on input a witness that contains indices $(2,3)$ and secret keys $\mathrm{Sk}_{2}, \mathrm{Sk}_{3}$.

Claim 5 The advantage of $\mathcal{A}$ in distinguishing $H_{4}^{N}$ from $H_{5}$ is negligible.

Proof. This follows straightforwardly from the WI property of NIWI ${ }^{\text {dec }}$. We note that both the randomness used to compute $\mathbf{S k}_{1}, \mathrm{Sk}_{3}$ and the randomness used to compute $\mathrm{Sk}_{2}, \mathrm{Sk}_{3}$ constitute valid witnesses for the statement $\left(\mathrm{Blt}_{1}, \ldots, \mathrm{Blt}_{N}\right.$, $\left.\mathcal{E} . \mathrm{Pk}_{1}, \ldots, \mathcal{E} . \mathrm{Pk}_{3}, y\right)$.

Hybrid $H_{6}^{k}$, for $k=0, \ldots, N$. For all $k=0, \ldots, N$, experiment $H_{6}^{k}$ is identical to experiment $H_{5}$ except that, for all $j=1, \ldots, k$, the challenger computes $\mathrm{Ct}_{k, 1}$ on input $m_{1, k}$. Note that $H_{6}^{0}$ is identical to $H_{5}$.

Claim 6 For all $k=1, \ldots, N$, the advantage of $\mathcal{A}$ in distinguishing $H_{6}^{k-1}$ from $H_{6}^{k}$ is negligible.

Proof. The proof is identical to the one for Claim 2 except that the third index and the first index are swapped.

Hybrid $H_{7}$. Experiment $H_{7}$ is identical to experiment $H_{6}^{N}$ except that the challenger computes the proof $\gamma$ on input a witness that contains indices $(1,2)$ and secret keys $\left(\mathrm{Sk}_{1}, \mathrm{Sk}_{2}\right)$.

Claim 7 The advantage of $\mathcal{A}$ in distinguishing $H_{6}^{N}$ from $H_{7}$ is negligible.

Proof. This follows straightforwardly from the WI property of NIWI dec . We note that both the randomness used to compute $\mathbf{S} \mathbf{k}_{1}, \mathbf{S k}_{2}$ and the randomness used to compute $\mathrm{Sk}_{2}, \mathrm{Sk}_{3}$ constitute valid witnesses for the statement $\left(\mathrm{Blt}_{1}, \ldots, \mathrm{Blt}_{N}\right.$, $\left.\mathcal{E} . \mathrm{Pk}_{1}, \ldots, \mathcal{E} . \mathrm{Pk}_{3}, y\right)$. 
Experiment $H_{1}\left(\right.$ resp. $\left.H_{7}\right)$ is identical to experiment WeakPriv ${ }_{\mathcal{A}}^{N, \mathcal{M}, \Sigma, F, \text { EVOTE }}$ except that the challenger sets $b=0$ (resp. $b=1$ ). Hence $\operatorname{Adv}_{\mathcal{A}}^{\text {EVOTE, WeakPriv }}\left(1^{\lambda}\right)$ equals at most the sum of the advantages of $\mathcal{A}$ in distinguishing the previous hybrids. Since $N$ is a constant, such advantage is negligible and the theorem is proven.

Corollary 3 If the Decision Linear assumption (see Section 3) holds, then there exists a weakly verifiable eVote.

Proof. Boneh et al. BBS04 show the existence of a PKE scheme with perfect correctness and unique secret key that fulfills the IND-CPA property under the Decision Linear assumption. Groth et al. GOS06 show the existence of (onemessage) NIWI proofs with perfect soundness for all languages in NP that is secure under the Decision Linear assumption. Then, because Theorem 1 and Theorem 2 are proven, the corollary follows.

\section{Our (Fully) Verifiable eVote}

In this Section, we present an eVote scheme EVOTE full $_{\text {that }}$ is IND-Secure and (fully) verifiable.

Definition 11 [EVOTE full $_{\text {li }}$ Let $\mathcal{E}=(\mathcal{E}$.Setup, $\mathcal{E}$.Encrypt, $\mathcal{E}$.Decrypt $)$ be a PKE scheme with perfect correctness and unique secret key (see Def. 66). Let Com be a perfectly binding commitment scheme. Let NIWI ${ }^{\text {dec,full }}=\left(\right.$ Prove $^{\text {dec,full }}$, Verify $\left.{ }^{\text {dec,full }}\right)$ and NIWI ${ }^{\text {enc,full }}=\left(\right.$ Prove $^{\text {enc,full }}$, Verify $\left.{ }^{\text {enc,full }}\right)$ be two NIWI proof systems for the relations $\mathrm{R}^{\text {dec,full }}$ and $\mathrm{R}^{\text {enc,full }}$, which we specify later.

We define as follows an $(N, \mathcal{M}, \Sigma, F)$-eVote

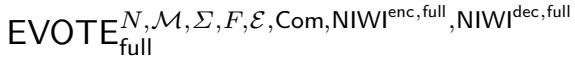

$$
\begin{aligned}
& =\left(\text { Setup }_{\text {full }}, \text { Cast }_{\text {full }}, \text { VerifyBallot } \text { full }, \text { EvalTally }_{\text {full }}, \text { Verify Tally }_{\text {full }}\right)
\end{aligned}
$$

- $\operatorname{Setup}_{\text {full }}\left(1^{\lambda}\right)$ : on input the security parameter in unary, do the following.

1. Choose randomness $r \leftarrow\{0,1\}^{\lambda}$ and set $Z=\operatorname{Com}(1 ; r)$.

2. For all $l \in[3]$, choose randomness $s_{l} \leftarrow\{0,1\}^{\lambda}$ and $\operatorname{run}\left(\mathcal{E}\right.$. $\left.\mathrm{Pk}_{l}, \mathcal{E} . \mathrm{Sk}_{l}\right)=$ $\mathcal{E}$.Setup $\left(1^{\lambda} ; s_{l}\right)$.

3. Output $\mathrm{Pk} \triangleq\left(\mathcal{E} . \mathrm{Pk}_{1}, \ldots, \mathcal{E} . \mathrm{Pk}_{3}, Z\right)$ and $\mathrm{Sk} \triangleq\left(\mathcal{E} . \mathrm{Sk}_{1}, \mathcal{E} . \mathrm{Sk}_{2}, s_{1}, s_{2}, r\right) 10$

- Cast $_{\text {full }}(\mathrm{Pk}, j, v)$ : on input the public key $\mathrm{Pk}$, the voter index $j \in[N]$, and a vote $v$, do the following.

1. For all $l \in[3]$, choose randomness $r_{l} \leftarrow\{0,1\}^{\lambda}$ and compute $\mathrm{Ct}_{j, l}=$ $\mathcal{E}$. Encrypt $\left(\mathcal{E}\right.$.Pk $\left.\mathrm{k}_{l}, v ; r_{l}\right)$.

2. Consider the following relation $\mathrm{R}^{\text {enc,full }}$ in Fig. 5. Run Prove ${ }^{\text {enc,full }}$ on input the statement $\left(j, \mathrm{Ct}_{1}, \ldots, \mathrm{Ct}_{3}, \mathcal{E} \cdot \mathrm{Pk}_{1}, \ldots, \mathcal{E} \cdot \mathrm{Pk}_{3}, Z\right)$ and the witness $\left(v, r_{1}, \ldots, r_{3}\right)$ to compute a proof $\pi_{j}$. Output Blt $\mathrm{t}_{j} \triangleq\left(\mathrm{Ct}_{j, 1}, \ldots, \mathrm{Ct}_{j, 3}, \pi_{j}\right)$.

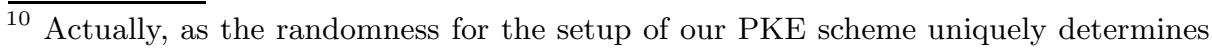
the secret key, it would be sufficient to just include the $s_{l}$ 's in Sk. 
Relation $\mathrm{R}^{\text {enc, full }}(x, w)$ :

Instance: $x \triangleq\left(j, \mathrm{Ct}_{1}, \ldots, \mathrm{Ct}_{3}, \mathcal{E} . \mathrm{Pk}_{1}, \ldots, \mathcal{E} \cdot \mathrm{Pk}_{3}, Z\right)$.

Witness : $w \triangleq\left(m, r_{1}, \ldots, r_{3}, u\right)$, where the $r_{l}$ 's are the randomness used to compute the ciphertexts $\mathrm{Ct}_{l}$ 's and $u$ is the randomness used to compute the commitment $Z$.

$\mathrm{R}^{\text {enc,full }}(x, w)=1$ if and only if either of the following two conditions hold:

(a) Real mode. All 3 ciphertexts $\left(\mathrm{Ct}_{1}, \ldots, \mathrm{Ct}_{3}\right)$ encrypt the same string in $\mathcal{M} \cup$ $\{\perp\}$.

Precisely, for all $l \in[3], \mathrm{Ct}_{l}=\mathcal{E}$.Encrypt $\left(\mathcal{E} . \mathrm{Pk}_{l}, m ; r_{l}\right)$ and $m \in \mathcal{M} \cup\{\perp\}$.

OR

(b) Trapdoor mode. $Z$ is a commitment to 0 .

Precisely, $Z=\operatorname{Com}(0 ; u)$.

Fig. 5. Relation $\mathrm{R}^{\text {enc, full }}$

- VerifyBallot full $_{\text {(I }}(\mathrm{Pk}, j, \mathrm{Blt})$ : on input the public key $\mathrm{Pk}$, the voter index $j \in[N]$, and a ballot Blt, output Verify ${ }^{\text {enc, full }}\left(\left(j, \mathrm{Ct}_{1}, \ldots, \mathrm{Ct}_{3}, \mathcal{E} . \mathrm{Pk}_{1}, \ldots, \mathcal{E} . \mathrm{Pk}_{3}, Z\right), \pi\right)$.

- EvalTally full $\left(\mathrm{Pk}, \mathrm{Sk}, \mathrm{Blt}, \ldots, \mathrm{Blt}_{N}\right)$ : on input the public key Pk, the secret key Sk, and $N$ strings $\left(\mathrm{Blt}_{1}, \ldots, \mathrm{Blt}_{N}\right)$ that can be either ballots cast by a voter or the special symbol $\perp$, do the following.

1. For all $j \in[N]$, if VerifyBallot full $_{\text {I }}\left(\mathrm{Pk}, j, \mathrm{Blt}_{j}\right)=\perp$, set $\mathrm{Blt}_{j}=\perp$. If, for all $j \in[N], \mathrm{Blt}_{j}=\perp$, then output $(y=\perp, \gamma=\perp)$.

2. Else, for all $j \in[N], l \in[2]$,

$$
m_{j}^{l}= \begin{cases}\perp & \text { if Blt }_{j}=\perp, \\ \perp & \text { if Blt } t_{j} \neq \perp \wedge \mathcal{E} . \operatorname{Decrypt}\left(\mathrm{Ct}_{j, l}, \mathcal{E} . \mathrm{Sk}_{l}\right) \notin \mathcal{M}, \\ \mathcal{E} . \operatorname{Decrypt}\left(\mathrm{Ct}_{j, l}, \mathcal{E} . \mathrm{Sk}_{l}\right) & \text { otherwise. }\end{cases}
$$

3. For all $l \in[2]$, compute $y_{l}=F\left(m_{1, l}, \ldots, m_{N, l}\right)$.

4. If $y_{1}=y_{2}$ then set $y=y_{1}$.

5. Consider the following relation $R^{\text {dec,full }}$ in Fig. 6] (The relation $R^{\text {dec,full }}$ is identical to the relation $R^{\text {dec }}$ that is used in our weakly verifiable eVote. The only difference is that the ballots in the statement of $R^{\text {dec,full }}$ are replaced by $\perp$ if they are not accepted by the ballot verification algorithm. Henceforth, if the indices $\left(i_{1}, i_{2}\right)$ in the witness of the relation $\mathrm{R}^{\text {dec }}$ fulfill $i_{1}=1$ and $i_{2}=2$ (resp. $i_{1} \neq 1$ or $i_{2} \neq 2$ ), the statement or the proof is in real mode (resp. trapdoor mode).) Run Prove ${ }^{\text {dec, full }}$ on input the statement $\left(\mathrm{Blt}_{1}, \ldots, \mathrm{Blt}_{N}, \mathcal{E}\right.$. $\left.\mathrm{Pk}_{1}, \ldots, \mathcal{E} . \mathrm{Pk}_{3}, y\right)$ and the witness $\left(\mathcal{E} . \mathrm{Sk}_{1}, \mathcal{E} . \mathrm{Sk}_{2}, s_{1}, s_{2}, i_{1}=1, i_{2}=2\right)$ to compute a proof $\gamma$. 
Relation $\mathrm{R}^{\text {dec,full }}(x, w)$ :

Instance: $x \triangleq\left(\mathrm{Blt}_{1}, \ldots, \mathrm{Blt}{ }_{N}, \mathcal{E} \cdot \mathrm{Pk}_{1}, \ldots, \mathcal{E} \cdot \mathrm{Pk}_{3}, y\right)$. (Recall that a ballot is set to $\perp$ if either the corresponding voter did not cast her vote or her ballot is not accepted by the ballot verification algorithm.)

Witness: $w \triangleq\left(\mathcal{E} . \mathrm{Sk}_{1}^{\prime}, \mathcal{E} . \mathrm{Sk}_{2}^{\prime}, s_{1}, s_{2}, i_{1}, i_{2}\right)$, where the $s_{l}$ 's are the randomness used to generate the secret keys and public keys (which are known to the authority who set up the system).

$\mathrm{R}^{\text {dec,full }}(x, w)=1$ if and only if the following condition holds.

2 of the secret keys corresponding to indices $\mathcal{E} . \mathrm{Pk}_{i_{1}}, \mathcal{E} . \mathrm{Pk}_{i_{2}}$ are constructed using honestly generated public and secret key pairs and are equal to $\mathcal{E} . \mathrm{Sk}_{1}^{\prime}, \mathcal{E} . \mathrm{Sk}_{2}^{\prime}$; and either $y=\perp$ or for all $l \in[2], y=F\left(m_{1}^{l}, \ldots, m_{N}^{l}\right)$ and for all $j \in[N]$, if $\mathrm{Blt}_{j} \neq \perp$ then for $l \in[2], \mathcal{E} . \mathrm{Sk}_{i_{l}}$ decrypts ciphertext $\mathrm{Ct}_{j, i_{l}}$ in $\mathrm{Blt}_{j}$ to $m_{j}^{i_{l}} \in \mathcal{M}$; and for all $l \in[2], m_{j}^{l}=\perp$ if either Blt $\mathrm{B}_{j}=\perp$ or $\mathcal{E} . \mathrm{Sk}_{i_{l}}$ decrypts $\mathrm{Ct}_{j, i_{l}}$ to a string $\notin \mathcal{M}$.

Precisely, $\mathrm{R}^{\text {dec,full }}(x, w)=1$ if and only if the following conditions hold. In the following, items (a) and (c) are not actually conditions that have to be checked but are steps needed to define (note the use of " $\triangleq$ ") the variables $\mathcal{E} . \mathrm{Pk}_{i_{l}}$ 's, $\mathcal{E} . \mathrm{Sk}_{i_{l}}$ 's and $m_{j}^{i_{l}}$ 's that are used in the checks (b) and (d).

(a) For all $l \in[2],\left(\mathcal{E} \cdot \mathrm{Pk}_{i_{l}}, \mathcal{E} \cdot \mathrm{Sk}_{i_{l}}\right) \triangleq \mathcal{E} \cdot \operatorname{Setup}\left(1^{\lambda} ; s_{l}\right)$.

(b) For all $l \in[2], \mathcal{E} . \mathrm{Sk}_{l}^{\prime}=\mathcal{E} . \mathrm{Sk}_{i_{l}}$.

(c) For all $j \in[N], l \in[2]$,

$$
m_{j}^{i_{l}} \triangleq \begin{cases}\perp & \text { if } \mathrm{Blt}_{j}=\perp \\ \perp & \text { if Blt } \neq \perp \wedge \mathcal{E} . \operatorname{Decrypt}\left(\mathrm{Ct}_{j, i_{l}}, \mathcal{E} . \mathrm{Sk}_{i_{l}}\right) \notin \mathcal{M} \\ \mathcal{E} . \operatorname{Decrypt}\left(\mathrm{Ct}_{j, i_{l}}, \mathcal{E} . \mathrm{Sk}_{i_{l}}\right) & \text { otherwise. }\end{cases}
$$

(d) $(y=\perp) \vee\left(\right.$ for all $\left.l \in[2], y=F\left(m_{1}^{i_{l}}, \ldots, m_{N}^{i_{l}}\right)\right)$.

(Note that $\mathcal{E} . \mathrm{Sk}_{1}^{\prime}$ and $\mathcal{E}$.Sk ${ }_{2}^{\prime}$ do not necessarily have to correspond to the first two secret keys.)

Fig. 6. Relation $R^{\text {dec,full }}$ 
6. Output $(y, \gamma)$.

- Verify Tally full $\left(\mathrm{Pk} \mathrm{Blt}_{1}, \ldots, \mathrm{Blt}_{N}, y, \gamma\right)$ : on input the public key $\mathrm{Pk}, N$ strings that can be either ballots cast by a voter or the special symbol $\perp$, a tally $y$ and a proof $\gamma$ of tally correctness, do the following. If $y=\perp$ and all Blt ${ }_{j}$ 's are equal to $\perp$, output OK. If $y=\perp$ but not all Blt ${ }_{j}$ 's are equal to $\perp$, output $\perp$. Otherwise output the decision of Verify ${ }^{\text {dec, full }}\left(\left(\mathrm{Blt}_{1}, \ldots, \mathrm{Blt}_{N}, \mathcal{E} . \mathrm{Pk}_{1}, \ldots\right.\right.$, $\mathcal{E}$.Pk $\left.{ }_{3}, y\right), \gamma$ ), after having replaced Blt ${ }_{j}$ 's with $\perp$ when VerifyBallot full $_{1}(\mathrm{Pk}, j$, $B\left(t_{j}\right)=\perp$. Precisely, the algorithm does the following:

1. For all $j \in[N]$, if VerifyBallot full $\left(\mathrm{Pk}, j, \mathrm{Blt}_{j}\right)=\perp$, set $\mathrm{Blt}_{j}=\perp$.

2. If $y \neq \perp$, then output Verify ${ }^{\text {dec,full }}\left(\left(\mathrm{Blt}_{1}, \ldots, \mathrm{Blt}_{N}, \mathcal{E} . \mathrm{Pk}_{1}, \ldots, \mathcal{E} . \mathrm{Pk}_{3}, y\right), \gamma\right)$.

3. If $y=\perp$, then, if for all $j \in[N], \mathrm{Blt}_{j}=\perp$, output OK, else output $\perp$.

Henceforth, for simplicity we omit the parameters of the scheme and we just write $\mathrm{EVOTE}_{\text {full. }}$.

\subsection{Correctness and (Full) Verifiability of the Construction}

Correctness. Condition (1) of (perfect) correctness of EVOTE the perfect correctness of the PKE scheme and the perfect completeness of NIWI ${ }^{\text {dec,full }}$ and NIW $\left.\right|^{\text {enc,full }}$. Condition (2) follows analogously. We note the following. For all honestly computed $\mathrm{Pk}, \mathrm{Pk}=\left(\mathrm{Pk}_{1}, \mathrm{Pk}_{2}, \mathrm{Pk}_{3}, Z\right)$ holds for some $\mathrm{Pk}_{1}, \mathrm{Pk}_{2}, \mathrm{Pk}_{3}$ and $Z . Z$ is a commitment to 1 . Therefore, relation $\mathrm{R}^{\text {enc,full }}$ and the perfectly binding property of the commitment scheme imply that, if there exists a proof $\pi$ and a statement $x=\left(j, \mathrm{Ct}_{1}, \ldots, \mathrm{Ct}_{3}, \mathrm{Pk}_{1}, \ldots, \mathrm{Pk}_{3}, Z\right)$ such that VerifyBallot full accepts $(x, \pi)$, then it must be the case that $\mathrm{Ct}_{1}, \ldots, \mathrm{Ct}_{3}$ encrypt the same string in $\mathcal{M} \cup\{\perp\}$. For all $j \in[N]$, if $\mathrm{Blt}_{j}$ is accepted by VerifyBallot full $_{1}, \mathrm{Blt}_{j}^{\prime}=\mathrm{Blt}_{j}$, else Blt ${ }_{j}^{\prime}=\perp$. Therefore, for all Blt ${ }_{1}, \ldots, \mathrm{Blt}_{N}$, if $(y, \gamma)=$ EvalTally $_{\text {full }}\left(\mathrm{Pk}, \mathrm{Blt}_{1}, \ldots, \mathrm{Blt}_{N}\right)$, then $y=F\left(m_{1}, \ldots, m_{N}\right)$, where, for all $j \in[N]$, if $\mathrm{Blt}_{j}$ is accepted by VerifyBallot full $_{1}, m_{j}$ is the string encrypted in the first two ciphertexts of $\mathrm{Blt}_{j}$, else $m_{j}$ is $\perp$. Then, it is easy to see that VerifyTally $_{\text {full }}\left(\mathrm{Pk}, \mathrm{Blt}_{1}, \ldots, \mathrm{Blt}_{N}, y, \gamma\right)=\mathrm{OK}$.

\section{(Full) verifiability.}

Theorem 4 For all $N>0$, all sets $\mathcal{M}, \Sigma \subset\{0,1\}^{\star}$, and all tally functions $F:(\mathcal{M} \cup\{\perp\})^{N} \rightarrow \Sigma \cup\{\perp\}$, if $\mathcal{E}$ is a perfectly correct PKE scheme with unique secret key (cf. Def. 6) , Com is a PPT algorithm, and NIWI dec,full $_{\text {and NIW }}^{\text {enc,full }}$ are (one-message) NIWIs (cf. Def. 91), for the relations $\mathrm{R}^{\text {dec,full }}$ and $\mathrm{R}^{\text {enc,full }}$ respec-

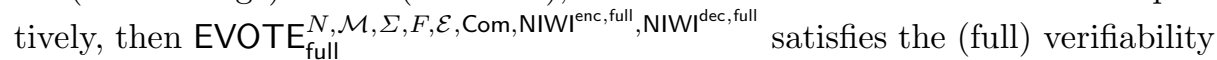
property (cf. Def. 3).

Proof. We first prove that condition (1) of verifiability is satisfied. We have to prove that, for all $\mathrm{Pk} \in\{0,1\}^{\star}$, and all $\mathrm{Blt}_{1}, \ldots, \mathrm{Blt}_{N} \in\{0,1\}^{\star} \cup\{\perp\}$ such that, for all $j \in[N]$, either $\mathrm{Blt}_{j}=\perp$ or VerifyBallot full $_{\text {II }}\left(\mathrm{Pk}, j, \mathrm{Blt}_{j}\right)=\mathrm{OK}$, there exist $m_{1}, \ldots, m_{N} \in \mathcal{M} \cup\{\perp\}$ such that, for all $y, \gamma \in\{0,1\}^{\star}$, if VerifyTally full $_{\text {(Pk, }}$ Blt ${ }_{1}$, $\left.\ldots, \mathrm{Blt}_{N}, y, \gamma\right)=1$ then $y=F\left(m_{1}, \ldots, m_{N}\right)$. Henceforth, w.l.o.g, we let Pk and 
$\mathrm{Blt}_{1}, \ldots, \mathrm{Blt}_{N}$ be arbitrary strings such that, for all $j \in[N]$, either $\mathrm{Blt}_{j}=\perp$ or VerifyBallot $_{\text {full }}\left(\mathrm{Pk}, j, \mathrm{Blt}_{j}\right)=\mathrm{OK}$.

First, we prove the following claim.

Claim 8 Given $\mathrm{Pk}$ and $\left(\mathrm{Blt}_{1}, \ldots, \mathrm{Blt}_{N}\right)$, for every two pairs $\left(y_{0}, \gamma_{0}\right)$ and $\left(y_{1}, \gamma_{1}\right)$, if VerifyTally full $_{\text {f }}\left(\mathrm{Pk} \mathrm{Blt}_{1}, \ldots, \mathrm{Blt}_{N}, y_{0}, \gamma_{0}\right)=$ VerifyTally $_{\mathrm{full}}\left(\mathrm{Pk}, \mathrm{Blt}_{1}, \ldots, \mathrm{Blt}_{N}, y_{1}\right.$, $\left.\gamma_{1}\right)=\mathrm{OK}$ then $y_{0}=y_{1}$.

For every $\left(y_{0}, \gamma_{0}\right)$ and $\left(y_{1}, \gamma_{1}\right)$, we have two cases.

1. Either $y_{0}=\perp$ and $y_{1} \neq \perp$ or $y_{1}=\perp$ and $y_{0} \neq \perp$. Suppose w.l.o.g. that $y_{0}=\perp$ and $y_{1} \neq \perp$. The other case (i.e., $y_{1}=\perp$ and $y_{0} \neq \perp$ ) is symmetrical. By construction, for all $(y, \gamma)$, it holds that $(\mathrm{A})$ if $\mathrm{Blt}_{1}=\cdots=\mathrm{Blt}_{N}=\perp$, then Verify Tally $_{\text {full }}\left(\mathrm{Pk} \mathrm{Blt}_{1}, \ldots, \mathrm{Blt}_{N}, y, \gamma\right)=\mathrm{OK}$ if and only if $y=\perp$ and (B) if, for some $j \in[N], \mathrm{Blt}_{j} \neq \perp$, then Verify Tally full $_{\text {f }}\left(\mathrm{Pk} \mathrm{Blt}_{1}, \ldots, \mathrm{Blt}_{N}, \perp, \gamma\right)=\perp$. We now have two cases.

(a) $\mathrm{Blt}_{1}=\cdots=\mathrm{Blt}_{N}=\perp$. Then we have that Verify Tally $\mathrm{full}_{\mathrm{fl}}\left(\mathrm{Pk}, \mathrm{Blt}_{1}, \ldots\right.$, $\left.\mathrm{Blt}_{N}, y_{1}, \gamma_{1}\right)=\mathrm{OK}$ and by (A) $y_{1}=\perp$, which is a contradiction.

(b) It is not the case that $\mathrm{Blt}_{1}=\cdots=\mathrm{Blt}_{N}=\perp$. Then, by (B) we have that VerifyTally full $\left(\mathrm{Pk}, \mathrm{Blt}_{1}, \ldots, \mathrm{Blt}_{N}, y_{0}, \gamma_{0}\right)=\perp$, which contradicts the fact that $\left(y_{0}, \gamma_{0}\right)$ is accepted.

2. $y_{0}, y_{1} \neq \perp$.

Let $y_{0}, \gamma_{0}, y_{1}, \gamma_{1}$ be arbitrary strings in $\{0,1\}^{\star} \cup\{\perp\}$ such that $y_{0}, y_{1} \neq$ $\perp$. Suppose that VerifyTally full $\left(\mathrm{Pk}, \mathrm{BIt}_{1}, \ldots, \mathrm{Blt}_{N}, y_{0}, \gamma_{0}\right)=$ Verify Tally $_{\text {full }}(\mathrm{Pk}$, $\left.\mathrm{Blt}_{1}, \ldots, \mathrm{Blt}_{N}, y_{1}, \gamma_{1}\right)=\mathrm{OK}$. The perfect soundness of NIWI dec,full implies that, for all $b \in\{0,1\}$, the proof $\gamma_{b}$ is computed on input some witness $\left(\mathcal{E} . \mathrm{Sk}_{1}^{\prime b}, \mathcal{E} . \mathrm{Sk}_{2}^{\prime b}, s_{1}^{b}, s_{2}^{b}, i_{1}^{b}, i_{2}^{b}\right)$.

By the pigeon principle, there exists an index $i^{\star}$ such that one of the following cases holds.

(a) $i^{\star}=i_{1}^{0}=i_{2}^{1}$. For all $b \in\{0,1\}$, let $\left(m_{1}^{i^{\star}, b}, \ldots, m_{N}^{i^{\star}, b}\right)$ be the messages guaranteed by condition (iii) of relation $\mathrm{R}^{\text {dec,full }}$ for proof $\gamma_{b}$. Condition (i) for proof $\gamma_{0}\left(\right.$ resp. $\left.\gamma_{1}\right)$ implies that the secret key $\mathbf{S k}_{1}^{\prime 0}\left(\right.$ resp. $\left.\mathbf{S k}_{2}^{\prime 1}\right)$ is honestly computed and thus, the unique secret key property and the fact that it fulfills $\mathcal{E} . \mathrm{Pk}_{i_{1}^{0}}=\mathcal{E} . \mathrm{Pk}_{i^{\star}}\left(\mathrm{resp} . \mathcal{E} \cdot \mathrm{Pk}_{i_{2}^{1}}=\mathcal{E} . \mathrm{Pk}_{i^{\star}}\right)$ imply that for all $j \in[N], \mathcal{E}$.Decrypt $\left(\mathrm{Ct}_{j, i^{\star}}, \mathcal{E}\right.$. Sk $\left.{ }_{1}^{\prime 0}\right)=\mathcal{E}$.Decrypt $\left(\mathrm{Ct}_{j, i^{\star}}, \mathcal{E}\right.$.S $\left.\mathrm{Sk}_{2}^{\prime 1}\right)$.

Furthermore, condition (ii) and (iii) for proof $\gamma_{0}$ (resp. $\gamma_{1}$ ) imply that for all $j \in[N]$, either $m_{j}^{i^{\star}, 0}=\perp$ or $m_{j}^{i^{\star}, 0}=\mathcal{E}$.Decrypt $\left(\mathrm{Ct}_{j, i^{\star}}, \mathcal{E} . \mathrm{Sk}_{1}^{\prime}\right) \in \mathcal{M}$ (resp. either $m_{j}^{i^{\star}, 1}=\perp$ or $m_{j}^{i^{\star}, 1}=\mathcal{E}$. Decrypt $\left.\left(\mathrm{Ct}_{j, i^{\star}}, \mathcal{E} . \mathrm{Sk}_{2}^{\prime 1}\right) \in \mathcal{M}\right)$.

Hence, for all $j \in[N], m_{j}^{i^{\star}, 0}=m_{j}^{i^{\star}, 1} \in \mathcal{M} \cup\{\perp\}$. Now, condition (iv) for proof $\gamma_{0}$ (resp. $\gamma_{1}$ ) implies that either $y_{0}=F\left(m_{1}^{i_{1}^{0}, 0}, \ldots, m_{N}^{i_{1}^{0}, 0}\right)$ or $y_{0}=\perp$ (resp. either $y_{1}=F\left(m_{1}^{i_{2}^{1}, 1}, \ldots, m_{N}^{i_{2}^{1}, 1}\right)$ or $y_{1}=\perp$ ) and, as by hypothesis $y_{0}, y_{1} \neq \perp$, it holds that $y_{0}=y_{1}$.

(b) $i^{\star}=i_{2}^{0}=i_{1}^{1}$. This case is identical to the first one, except that we replace $i_{1}^{0}$ with $i_{2}^{0}$ and $i_{2}^{1}$ with $i_{1}^{1}$.

(c) $i^{\star}=i_{1}^{0}=i_{1}^{1}$. This case is identical to the first one, except that we replace $i_{2}^{1}$ with $i_{1}^{1}$. 
(d) $i^{\star}=i_{2}^{0}=i_{2}^{1}$. This case is identical to the first one, except that we replace $i_{1}^{0}$ with $i_{2}^{0}$.

In all cases, if Verify Tally full $_{\text {( }}\left(\mathrm{Pk}, \mathrm{Blt}_{1}, \ldots, \mathrm{Blt}_{N}, y_{0}, \gamma_{0}\right)=$ Verify Tally $_{\text {full }}\left(\mathrm{Pk}, \mathrm{Blt}_{1}\right.$, $\left.\ldots, \mathrm{Blt}_{N}, y_{1}, \gamma_{1}\right)=\mathrm{OK}$ then $y_{0}=y_{1}$. In conclusion, the claim is proved.

From the previous claim, it follows that there exists a unique value $y^{\star}$ such that, for all $(y, \gamma)$ such that $y \neq \perp$, if Verify Tally full $\left(\mathrm{Pk}, \mathrm{Blt}_{1}, \ldots, \mathrm{Blt}_{N}, y, \gamma\right)=\mathrm{OK}$ then $y=y^{\star}(1)$. Moreover, it is easy to see that, for all $(y, \gamma)$, if Verify Tally full $_{\text {( }}(\mathrm{Pk}$, $\left.\mathrm{Blt}_{1}, \ldots, \mathrm{Blt}_{N}, y, \gamma\right)=\mathrm{OK}$, there exist messages $m_{1}, \ldots, m_{N} \in \mathcal{M} \cup\{\perp\}$ such that $y=F\left(m_{1}, \ldots, m_{N}\right)(2)$.

Now, we have two mutually exclusive cases.

- For all $(y, \gamma)$ such that $y \neq \perp$, VerifyTally full $_{\text {f }}\left(\mathrm{Pk}_{\mathrm{Blt}}, \ldots, \mathrm{Blt}_{N}, y, \gamma\right)=\perp$. Then, letting $m_{1}, \ldots, m_{N}$ in the statement of the theorem be arbitrary messages in $\mathcal{M} \cup\{\perp\}$, the statement is verified with respect to $\mathrm{Pk}$ and $\mathrm{Blt}_{1}, \ldots, \mathrm{Blt}_{N}$.

- There exists $\left(y^{\prime}, \gamma\right)$ such that $y^{\prime} \neq \perp$ and VerifyTally full $_{\text {f }}\left(\mathrm{Pk}_{\mathrm{B}} \mathrm{Blt}_{1}, \ldots, \mathrm{Blt}_{N}, y^{\prime}\right.$, $\gamma)=$ OK. In this case, (2) implies that there exist $m_{1}^{\prime}, \ldots, m_{N}^{\prime} \in \mathcal{M} \cup\{\perp\}$ such that $y^{\prime}=F\left(m_{1}^{\prime}, \ldots, m_{N}^{\prime}\right)(3)$. Hence, (1) and (3) together imply that $y^{\star}=F\left(m_{1}^{\prime}, \ldots, m_{N}^{\prime}\right)(4)$.

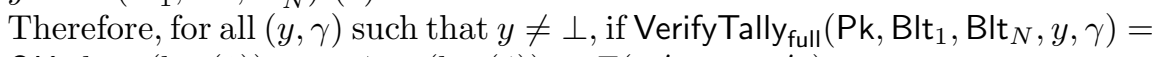
OK then (by (1)) $y=y^{\star}=($ by $(4))=F\left(m_{1}^{\prime}, \ldots, m_{N}^{\prime}\right)$.

Then, for $m_{1} \triangleq m_{1}^{\prime}, \ldots, m_{N} \triangleq m_{N}^{\prime}$, the statement of condition (1) of weak verifiability is verified with respect to $\mathrm{Pk}$ and $\mathrm{Blt}_{1}, \ldots, \mathrm{Blt}_{N}$.

In both cases, for $m_{1} \triangleq m_{1}^{\prime}, \ldots, m_{N} \triangleq m_{N}^{\prime}$, the statement of condition (1) of weak verifiability is verified with respect to $\mathrm{Pk}$ and $\mathrm{Blt}_{1}, \ldots, \mathrm{Blt}_{N}$.

As $\mathrm{Pk}$ and $\mathrm{Blt}_{1}, \ldots, \mathrm{Blt}_{N}$ are arbitrary strings, the statement of condition (1) of weak verifiability is proven.

It is also easy to check that condition (2) of weak verifiability is satisfied. This follows straightforwardly from the perfect soundness of NIWIdec,full. Thanks to $\mathrm{NIWI}^{\text {dec,full }}$, the authority always proves that the public key of the PKE scheme is honestly generated. Therefore, by the perfect correctness of the PKE scheme, an honestly computed ballot for message $m$ for the $j$-th voter is decrypted to $m$ (because an honestly computed ballot, by definition, consists of three ciphertexts that encrypt the same message, and thus the value committed to in $Z$ is not relevant). Consequently, if the tally $y$ is different from $\perp$ (i.e., if the evaluation of the tally function is equal for all indices), then $y$ has to be compatible with $m$ at index $j$ (cf. Def. 1).

In essence, condition (2) is satisfied because the degree of freedom of the authority in creating a dishonest public key only allows it to set up the commitment dishonestly. This does not affect how honest ballots are decrypted and "counted".

Note that, for the proof of the theorem above, the security of the commitment scheme Com is not needed, i.e., the theorem holds for any PPT algorithm Com, even insecure ones. 


\subsection{Privacy of the Construction}

Theorem 5 For all $N>0$, all sets $\mathcal{M}, \Sigma \subset\{0,1\}^{\star}$, and all tally functions $F$ : $(\mathcal{M} \cup\{\perp\})^{N} \rightarrow \Sigma \cup\{\perp\}$, if $\mathcal{E}$ is a perfectly correct PKE scheme with unique secret key (cf. Def. 6), Com is a computationally hiding commitment scheme (cf. Def. [7), and NIWI ${ }^{\text {dec,full }}$ and NIWI ${ }^{\text {enc,full }}$ are (one-message) NIWIs (cf. Def. 9), respectively, for the relations $\mathrm{R}^{\text {dec,full }}$ and $\mathrm{R}^{\text {enc,full }}$, then $\operatorname{EVOTE}_{\text {full }}^{N, \mathcal{M}, \Sigma, F, \mathcal{E}, \text { Com, NIWI }}$ enc,full, NIIIIde, full is IND-Secure (cf. Def. 4).

Proof. Consider the following experiment $H_{\mathcal{A}}^{Z}\left(1^{\lambda}\right)$ between a challenger and $\mathcal{A}$ (henceforth, we often omit the parameters).

Experiment $H^{Z} \cdot H^{Z}$ is equal to the experiment $\operatorname{Priv}_{\mathcal{A}}^{N, \mathcal{M}, \Sigma, F, \mathrm{EVOTE}_{\text {full }}}$ except that the challenger sets the commitment $Z$ in the public key to be a commitment to 0 instead of 1 . We define the output of the experiment to be a bit that is 1 if and only if all winning conditions are satisfied. Then, consider the following claim.

Claim 9 The probability $P_{0}$ that $\mathcal{A}$ wins the experiment $\operatorname{Priv}_{\mathcal{A}}^{N, \mathcal{M}, \Sigma, F, \text { EVOTE full }}$ is negligibly different from the probability $P_{1}$ that $\mathcal{A}$ wins game $H^{Z}$.

Proof. Suppose towards a contradiction that the difference between $P_{0}$ and $P_{1}$ is some non-negligible function $\epsilon(\lambda)$. We construct an adversary $\mathcal{B}$ that breaks the computationally hiding property of Com with non-negligible probability.

$\mathcal{B}$ receives as input a commitment com that is either a commitment to 0 or to 1 . For $l \in[3], \mathcal{B}$ runs $\mathcal{E}$.Setup $\left(1^{\lambda}\right)$ to compute $\left(\mathcal{E}\right.$. $\mathrm{Pk}_{l}, \mathcal{E}$. $\left.\mathrm{Sk}_{l}\right)$ and sets the public key $\mathrm{Pk}=\left(\mathcal{E} . \mathrm{Pk}_{1}, \ldots, \mathcal{E} \cdot \mathrm{Pk}_{3}, Z=\mathrm{com}\right) \cdot \mathcal{B}$ follows the challenger of $\operatorname{Priv}_{\mathcal{A}}^{N, \mathcal{M}, \Sigma, F, \text { EVOTE full }}$ to compute the remaining messages that are sent to the adversary. Finally, $\mathcal{B}$ gets the output $b^{\prime}$ from $\mathcal{A}$. $\mathcal{B}$ outputs 1 if and only if all winning conditions are satisfied.

By hypothesis, if com is a commitment to 1 , the probability that $\mathcal{B}$ outputs 1 equals the probability that $\mathcal{A}$ wins in $\operatorname{Priv}_{\mathcal{A}}^{N, \mathcal{M}, \Sigma, F, \text { EVOTE full }}$, and if com is a commitment to 0 , the probability that $\mathcal{B}$ outputs 1 equals the probability that $\mathcal{A}$ wins in $H^{Z}$. Thus, the advantage of $\mathcal{B}$ in breaking the computational hiding property of Com is $\epsilon(\lambda)$, which contradicts the assumption that the commitment scheme is computationally hiding.

Before continuing with the proof, we would like to remark a subtle point. In the previous claim, we implicitly assumed that the adversary $\mathcal{B}$ is able to check all of the winning conditions efficiently. This is possible if $\mathcal{M}$ is efficiently enumerable and its cardinality, as well as the number of voters $N$, are constant in the security parameter. This could seem like resorting to "complexity leveraging" arguments. In fact, one could ask if our proof would break down if $N$ and $\mathcal{M}$ depend on the security parameter. However, the whole proof can be generalized to the case of $N$ and $|\mathcal{M}|$ polynomial in the security parameter by using the following observation. Let $A$ be the event that $\mathcal{A}$ submits challenges that satisfy the winning condition. Then, if the probability that $\mathcal{A}$ wins the $\operatorname{Priv}_{\mathcal{A}}^{N, \mathcal{M}, \Sigma, F, \text { EVOTE }}$ 
is non-negligible, then the event $A$ must occur with non-negligible probability and, conditioned on it, $\mathcal{A}$ wins with non-negligible probability as well. Therefore, the rest of the proof would follow analyzing the probability that $\mathcal{A}$ wins in the next hybrid experiments conditioned under the occurrence of the event that, in such experiments, $\mathcal{A}$ submit challenges satisfying the winning condition. As we will see now, a similar "conditioning" argument will be anyhow necessary for the rest of the proof.

Let $E^{1}$ be the event that, in experiment $H^{Z}, \mathcal{A}$ submits as challenge two tuples $M_{0}=\left(m_{0,1}, \ldots, m_{0, N}\right)$ and $M_{1}=\left(m_{1,1}, \ldots, m_{1, N}\right)$ and a set $S \subset[N]$ that fulfill the following condition: there exists $j \in S$ such that $m_{0, j}=m_{1, j}$ and, letting $B=m_{0, j}=\left(\mathrm{Ct}_{1}, \ldots, \mathrm{Ct}_{3}\right)$ (suppose that $m_{0, j}$ can be parsed that way), it holds that VerifyBallot full $_{\text {(I }}(\mathrm{Pk}, j, B)=\mathrm{OK}$ but there exist $i_{1}, i_{2} \in[3], i_{1} \neq i_{2}$ such that $\mathcal{E}$.Decrypt $\left(\mathrm{Ct}_{i_{1}}, \mathrm{Sk}_{i_{1}}\right) \neq \mathcal{E}$.Decrypt $\left(\mathrm{Ct}_{i_{2}}, \mathrm{Sk}_{i_{2}}\right)$.

Claim 10 The probability that $E^{1}$ occurs is negligible.

Proof. Suppose towards a contradiction that the probability of occurrence of $E^{1}$ be some non-negligible function $\epsilon(\lambda)$. We construct an adversary $\mathcal{B}$ that breaks the computationally hiding property of Com with non-negligible probability.

$\mathcal{B}$ receives as input a commitment com that is either a commitment to 0 or to 1 . For $l \in[3], \mathcal{B}$ runs $\mathcal{E}$.Setup $\left(1^{\lambda}\right)$ to compute $\left(\mathcal{E}\right.$. $\mathrm{Pk}_{l}, \mathcal{E}$. $\left.\mathrm{Sk}_{l}\right)$ and sets the public key $\mathrm{Pk}=\left(\mathcal{E}\right.$. $\left.\mathrm{Pk}_{1}, \ldots, \mathcal{E} . \mathrm{Pk}_{3}, Z=\mathrm{com}\right) . \mathcal{B}$ follows the challenger of Priv $_{\mathcal{A}}^{N, \mathcal{M}, \Sigma, F, \text { EVOTE }}$ full to compute the remaining messages that are sent to the adversary. $\mathcal{B}$ receives two tuples $M_{0}=\left(m_{0,1}, \ldots, m_{0, N}\right)$ and $M_{1}=\left(m_{1,1}, \ldots, m_{1, N}\right)$ and a set $S \subset[N]$ from the adversary.

For all $j \in S, \mathcal{B}$ checks whether the following conditions are all satisfied: $m_{0, j}=m_{1, j}$ and, after setting $B=m_{0, j}, B$ can be parsed as $\left(\mathrm{Ct}_{1}, \ldots, \mathrm{Ct}_{3}\right)$ and it holds that VerifyBallot full $(\mathrm{Pk}, j, B)=\mathrm{OK}$ but there exist $i_{1}, i_{2} \in[3], i_{1} \neq i_{2}$ such that $\mathcal{E}$.Decrypt $\left(\mathrm{Ct}_{i_{1}}, \mathrm{Sk}_{i_{1}}\right) \neq \mathcal{E}$.Decrypt $\left(\mathrm{Ct}_{i_{2}}, \mathrm{Sk}_{i_{2}}\right)$. If for some $j \in S$ the conditions are satisfied, $\mathcal{B}$ outputs 0 , otherwise it outputs 1 .

If com is a commitment to 1 , the perfect soundness of NIWI ${ }^{\text {enc,full }}$ and the definition of relation $\mathrm{R}^{\text {enc,full }}$ guarantee that the conditions above are never satisfied for any $j \in S$. Therefore, if com is a commitment to $1 \mathcal{B}$ outputs 1 with probability 1 .

On the other hand, if com is a commitment to 0 , the probability that the conditions are satisfied for some $j \in[S]$ equals the probability of $E^{1}$. Therfore, $\mathcal{B}$ outputs 0 with probability $\epsilon$ and 1 with probability $1-\epsilon$. In conclusion, the advantage of $\mathcal{B}$ in breaking the computationally hiding property of Com is $\epsilon(\lambda)$, which contradicts the assumption that the commitment scheme is computationally hiding.

From Claim 9 and Claim 10, we now know that, for some negligible function $\operatorname{negl}(\cdot)$, the following equations hold:

$$
\begin{gathered}
\left|\operatorname{Pr}[\operatorname{Priv}=1]-\operatorname{Pr}\left[H^{Z}=1\right]\right| \leq \operatorname{negl}(\lambda), \\
\operatorname{Pr}\left[E^{1}\right] \leq \operatorname{negl}(\lambda),
\end{gathered}
$$




$$
\begin{array}{r}
\operatorname{Pr}\left[H^{Z}=1\right]=\operatorname{Pr}\left[H^{Z}=1 \mid E^{1}\right] \operatorname{Pr}\left[E^{1}\right]+\operatorname{Pr}\left[H^{Z}=1 \mid \bar{E}^{1}\right] \operatorname{Pr}\left[\bar{E}^{1}\right] \leq \\
\text { negl }+\operatorname{Pr}\left[H^{Z}=1 \mid \bar{E}^{1}\right](1-\text { negl }) .
\end{array}
$$

(Here and henceforth, we omit the parameters, but it is meant that the experiments are parameterized by $\lambda$ and negl(.).)

Thus, to show that $\operatorname{Pr}[\operatorname{Priv}=1]$ equals $1 / 2$ plus a negligible quantity, it is sufficient to show that $\operatorname{Pr}\left[H^{Z}=1 \mid \bar{E}^{1}\right]$ equals $1 / 2$ plus a negligible quantity. We prove the latter by means of a series of hybrid experiments. The reader could still refer to Table 1.5 for a pictorial explanation of the hybrid experiments. However, the experiments in the table, though conceptually very similar, correspond to the security reduction for the weakly verifiable eVote. Moreover, in the following we analyze the behavior of the adversary conditioned on the occurrence of the event $\bar{E}^{1}$.

Hybrid $H_{1}$. Experiment $H_{1}$ is equal to the experiment $H^{Z}$ except that the challenger sets $b=0$.

Hybrid $H_{2}^{k}$, for $k=0, \ldots, N$. For all $k=0, \ldots, N$, experiment $H_{2}^{k}$ is identical to experiment $H_{1}$ except that, for all $j=1, \ldots, k$ such that $j \notin S$, the challenger computes $\mathrm{Ct}_{k, 3}$ on input $m_{1, k}$. Note that $H_{2}^{0}$ is identical to $H_{1}$.

Claim 11 For all $k=1, \ldots, N,\left|\operatorname{Pr}\left[H_{2}^{k-1}=1 \mid \bar{E}^{1}\right]-\operatorname{Pr}\left[H_{2}^{k}=1 \mid \bar{E}^{1}\right]\right|$ is negligible.

Proof. Suppose toward a contradiction that the difference between such probabilities is non-negligible function $\epsilon(\lambda)$. We construct an adversary $\mathcal{B}$ that has advantage at most $\epsilon(\lambda)$ against the IND-CPA security of $\mathcal{E}$.

$\mathcal{B}$ receives from the challenger of IND-CPA a public key pk and sets $\mathrm{Pk}_{3}=\mathrm{pk}$. For $l \in[2], \mathcal{B}$ runs $\mathcal{E}$.Setup to compute $\left(\mathcal{E}\right.$. $\left.\mathrm{Pk}_{l}, \mathcal{E} . \mathrm{Sk}_{l}\right)$, computes $Z \leftarrow \operatorname{Com}(0)$ and runs $\mathcal{A}$ on input $\mathrm{Pk}=\left(\mathcal{E} . \mathrm{Pk}_{1}, \mathcal{E} \cdot \mathrm{Pk}_{2}, \mathcal{E} \cdot \mathrm{Pk}_{3}, Z\right)$.

$\mathcal{A}$ outputs two tuples $\left(m_{0,1}, \ldots, m_{0, N}\right)$ and $\left(m_{1,1}, \ldots, m_{1, N}\right)$ and a set $S$. If $k \in S, \mathcal{B}$ sends $(0,0)$ as its pair of challenge messages to the IND-CPA challenger, which returns the challenge ciphertext $\mathrm{ct}^{\star}$ to $\mathcal{B}$. If $k \notin S, \mathcal{B}$ sends $\left(m_{0, k}, m_{1, k}\right)$ as its pair of challenge messages to the IND-CPA challenger, which returns the challenge ciphertext $\mathrm{ct}^{\star}$ to $\mathcal{B}$.

If $k \in S, \mathcal{B}$ sets $\mathrm{Blt}_{j}$ as the challenger in the real experiment would do, else $\mathcal{B}$ computes $\mathrm{Blt}_{k}=\left(\mathrm{Ct}_{k, 1}, \mathrm{Ct}_{k, 2}, \mathrm{ct}^{\star}\right)$ by computing $\mathrm{Ct}_{k, 1}$ and $\mathrm{Ct}_{k, 2}$ on input $m_{0, j}$. For all $j \in[N](j \neq k), \mathcal{B}$ computes the ballots Blt ${ }_{j}$ exactly as the challenger in both experiments would do. $\mathcal{B}$ computes $y$ using EvalTally full and uses the 2 secret keys $\mathcal{E} . \mathrm{Sk}_{1}, \mathcal{E} . \mathrm{Sk}_{2}$ to compute a proof $\gamma$ exactly as the challenger in both experiments would do. $\mathcal{B}$ sends $\mathcal{A}$ the computed ballots along with $(y, \gamma)$ and returns the output of $\mathcal{A}$.

It is easy to see that, if $\mathrm{ct}^{\star}$ is an encryption of $m_{0, k}$ and if $k \notin S$, then $\mathcal{B}$ simulates experiment $H_{2}^{k-1}$ and if $\mathrm{ct}^{\star}$ is an encryption of $m_{1, k}$ and $k \notin S$, then $\mathcal{B}$ simulates experiment $H_{2}^{k}$. If $k \in S$ the advantage of $\mathcal{A}$ is 0 . 
Therefore, $\mathcal{B}$ has non-negligible probability of winning the IND-CPA game, which contradicts the assumption that the PKE scheme fulfills the IND-CPA property.

Hybrid $H_{3}$. Experiment $H_{3}$ is identical to experiment $H_{2}^{N}$ except that the challenger computes the proof $\gamma$ on input a witness that contains indices $(1,3)$ and secret keys $\left(\mathrm{Sk}_{1}, \mathrm{Sk}_{3}\right)$ (precisely, the witness contains the randomness used to compute those secret keys, but henceforth, for simplicity, we omit this detail).

Claim $12\left|\operatorname{Pr}\left[H_{2}^{N}=1 \mid \bar{E}^{1}\right]-\operatorname{Pr}\left[H_{3}=1 \mid \bar{E}^{1}\right]\right|$ is negligible.

Proof. The proof follows from the WI property of NIWIdec,full. We observe that both the randomness used to compute $\left(\mathbf{S k}_{1}, \mathbf{S k}_{2}\right)$ and the randomness used to compute $\left(\mathrm{Sk}_{1}, \mathrm{Sk}_{3}\right)$ constitute valid witnesses for the statement $\left(\mathrm{Blt}_{1}, \ldots, \mathrm{Blt} \mathrm{t}_{N}\right.$, $\mathcal{E}$.P $\left.\mathrm{k}_{1}, \ldots, \mathcal{E} . \mathrm{Pk}_{3}, y\right)$. Additionally, we observe that, if event $\bar{E}^{1}$ occurs, any ballot in the set $S$ is in both experiments either replaced by $\perp$, if VerifyBallot full refuses it, or decrypted to the same value. Consequently, the tally is identical in both experiments.

Hybrid $H_{4}^{k}$, for $k=0, \ldots, N$. For all $k=0, \ldots, N$, experiment $H_{4}^{k}$ is identical to experiment $H_{3}$ except that, for all $j=1, \ldots, k$ such that $j \notin S$, the challenger computes $\mathrm{Ct}_{k, 2}$ on input $m_{1, k}$. Note that $H_{4}^{0}$ is identical to $H_{3}$.

Claim 13 For all $k=1, \ldots, N,\left|\operatorname{Pr}\left[H_{4}^{k-1}=1 \mid \bar{E}^{1}\right]-\operatorname{Pr}\left[H_{4}^{k}=1 \mid \bar{E}^{1}\right]\right|$ is negligible.

Proof. The proof is identical to the one for Claim 11 except that the third index and the second index are swapped.

Hybrid $H_{5}$. Experiment $H_{5}$ is identical to experiment $H_{4}^{N}$ except that the challenger computes the proof $\gamma$ on input a witness that contains indices $(2,3)$ and secret keys $\left(\mathrm{Sk}_{2}, \mathrm{Sk}_{3}\right)$.

Claim $14\left|\operatorname{Pr}\left[H_{4}^{N}=1 \mid \bar{E}^{1}\right]-\operatorname{Pr}\left[H_{5}=1 \mid \bar{E}^{1}\right]\right|$ is negligible.

Proof. This follows straightforwardly from the WI property of NIWI ${ }^{\text {dec,full }}$. We observe that both the randomness used to compute $\left(\mathrm{Sk}_{1}, \mathrm{Sk}_{3}\right)$ and the randomness used to compute $\left(\mathrm{Sk}_{2}, \mathrm{Sk}_{3}\right)$ constitute valid witnesses for the statement $\left(\mathrm{Blt}_{1}, \ldots, \mathrm{Blt}_{N}, \mathcal{E} . \mathrm{Pk}_{1}, \ldots, \mathcal{E} . \mathrm{Pk}_{3}, y\right)$. Additionally, we observe that, if event $\bar{E}^{1}$ occurs, any ballot in the set $S$ is in both experiments either replaced by $\perp$, if VerifyBallot $_{\text {full }}$ refuses it, or decrypted to the same value. Consequently, the tally is identical in both experiments. 
Hybrid $H_{6}^{k}$, for $k=0, \ldots, N$. For all $k=0, \ldots, N$, experiment $H_{6}^{k}$ is identical to experiment $H_{5}$ except that, for all $j=1, \ldots, k$ such that $j \notin S$, the challenger computes $\mathrm{Ct}_{k, 1}$ on input $m_{1, k}$. Note that $H_{6}^{0}$ is identical to $H_{5}$.

Claim 15 For all $k=1, \ldots, N,\left|\operatorname{Pr}\left[H_{6}^{k-1}=1 \mid \bar{E}^{1}\right]-\operatorname{Pr}\left[H_{6}^{k}=1 \mid \bar{E}^{1}\right]\right|$ is negligible.

Proof. The proof is identical to the one for Claim 11 except that the third index and the first index are swapped.

Hybrid $H_{7}$. Experiment $H_{7}$ is identical to experiment $H_{6}^{N}$ except that the challenger sets $b=1$ (so that the winning condition be computed differently) and computes the proof $\gamma$ on input a witness that contains indices $(1,2)$ and secret keys $\left(\mathrm{Sk}_{1}, \mathrm{Sk}_{2}\right)$.

Claim $16\left|\operatorname{Pr}\left[H_{6}^{N}=1 \mid \bar{E}^{1}\right]-\operatorname{Pr}\left[H_{7}=0 \mid \bar{E}^{1}\right]\right|$ is negligible.

Proof. The proof follows straightforwardly from the WI property of NIWI ${ }^{\text {dec,full }}$. We observe that both the randomness used to compute $\left(\mathbf{S k}_{1}, \mathbf{S k}_{2}\right)$ and the randomness used to compute $\left(\mathrm{Sk}_{2}, \mathrm{Sk}_{3}\right)$ constitute valid witnesses for the statement $\left(\mathrm{Blt}_{1}, \ldots, \mathrm{Blt}_{N}, \mathcal{E} . \mathrm{Pk}_{1}, \ldots, \mathcal{E} . \mathrm{Pk}_{3}, y\right)$. Additionally, we observe that, if event $\bar{E}^{1}$ occurs, any ballot in the set $S$ is in both experiments either replaced by $\perp$, if VerifyBallot $_{\text {full }}$ refuses it, or decrypted to the same value. Consequently, the tally is identical in both experiments.

Note that according to the proof received, an adversary against NIWI can emulate experiment $H_{6}^{N}$ or $H_{7}$, and return the output of $\mathcal{A}$. In the first case, the probability that $\mathcal{A}$ outputs 0 is exactly $\operatorname{Pr}\left[H_{6}^{N}=1 \mid \bar{E}^{1}\right]$ because the winning condition is computed with respect to $b=0$, whereas in the second case it is $\operatorname{Pr}\left[H_{7}=0 \mid \bar{E}^{1}\right]$ because the winning condition is computed with respect to $b=1$.

Now, consider Equation 4 in Fig. 7 . Finally, Claim 9 and equations 23 and 4 imply that $\operatorname{Pr}[\operatorname{Priv}=1] \leq \nu$ for some negligible function $\nu$ and the theorem is proven.

Corollary 6 If the Decision Linear assumption (see Section 3) holds, then there exists a (fully) verifiable eVote.

Proof. Boneh et al. [BBS04] show the existence of a PKE with perfect correctness and unique secret key that fulfills the IND-CPA property under the Decision Linear assumption. Groth et al. [GOS06] show the existence of (one-message) NIWI (with perfect soundness) for all languages in NP and of statistically binding commitments. Both constructions are secure under the Decision Linear assumption. Then, because Theorem 4 and Theorem 5 are proven, the corollary follows. 


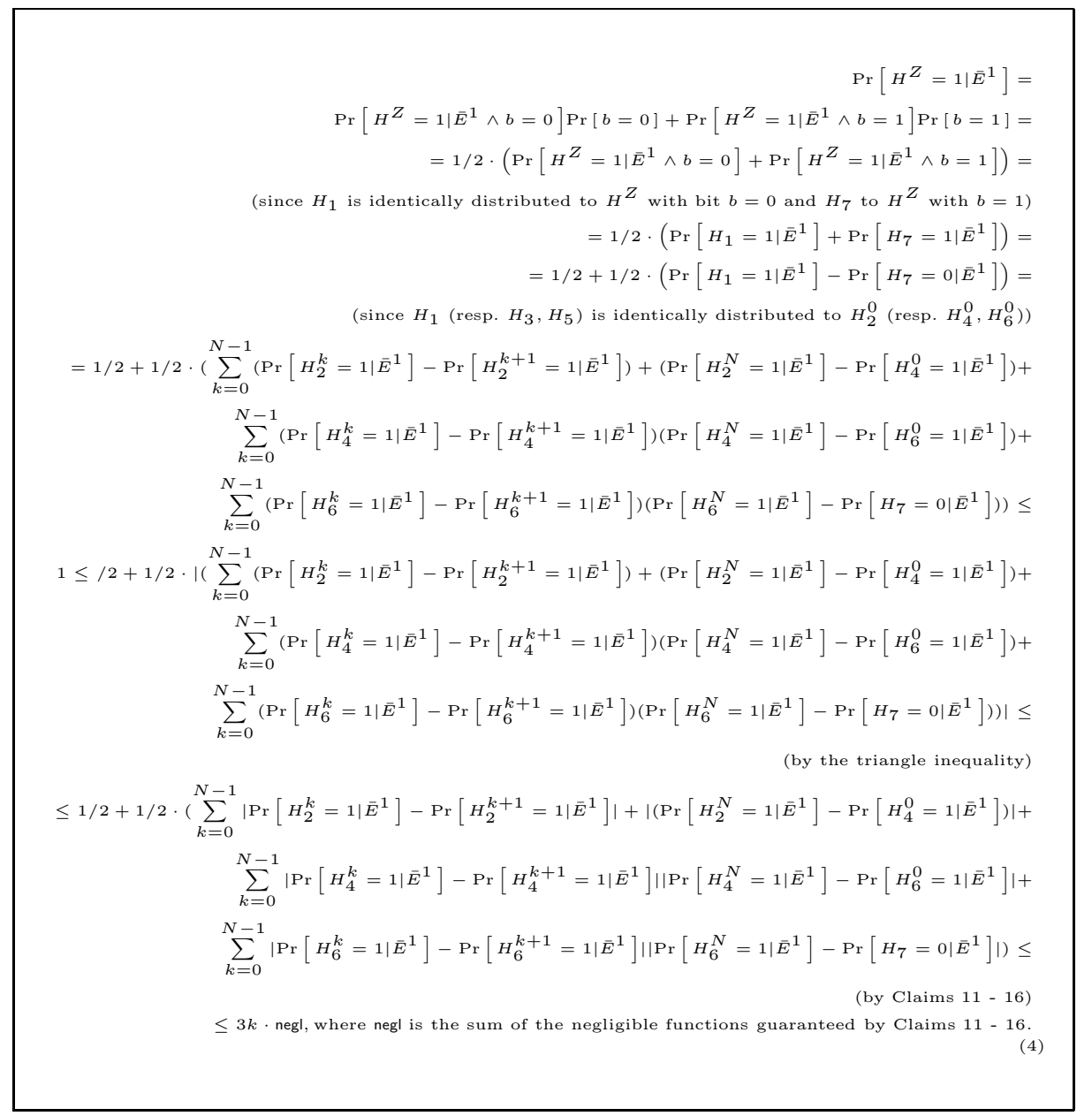

Fig. 7. Equation 4 


\section{$6 \quad$ Future Directions}

Our work opens up new directions in e-voting and generally in cryptography. We discuss some of them.

- Efficiency. In our work, in order to compute the NIWI proofs of Groth et al. GOS06 for CircuitSat, we need to represent the computation as a Boolean circuit and, though this can be done in polynomial time, it can be inefficient in practice. An important objective is to sidestep the reduction to circuits by employing a more direct approach. A possibility would be to explore the achievability of our results from variants of Groth-Sahai NIWIs GS08. The NIWI of Groth-Sahai, as it stands, is formulated in the CRS model but it is worthy to study in which settings it can be instantiated without CRS.

Another important direction is to improve the efficiency of verification. It would be desirable that the cost for verifiers be sub-linear in the number of voters. The verifiability guarantees attained would then be computational but hopefully it could be possible to avoid trust assumptions. A possibility would be to employ variants of succinct arguments (see [Bit14 for a survey).

- Receipt-freeness. Perfect verifiability and perfect correctness seem incompatible with receipt-freeness [BT94|SK95|MH96|MN06 DKR09 CCFG15, but we think that it should be possible to define a statistical variant of verifiability that could coexist with some form of receipt-freeness. Another possibility could be to resort to some voting server trusted for receipt-freeness but not for privacy that re-randomizes the ballots, as done in BeleniosRF of Chaidos, Cortier, Fuchsbauer and Galindo CCFG15.

- Other applications of our techniques. We think that our techniques could be of wide applicability to other settings. For instance, Camenisch and Shoup CS03a put forth the concept of verifiable encryption (that in some sense could be also viewed as a special case of verifiable functional encryption BGJS16) and present numerous applications of it, such as key escrow, optimistic fair exchange, publicly verifiable secret and signature sharing, universally composable commitments, group signatures, and confirmer signatures. We believe that our techniques can be employed profitably to improve their results with the aim of removing the need of trust assumptions.

\section{Acknowledgments}

Vincenzo Iovino thanks Saikrishna Badrinarayanan and Aayush Jain for helpful discussions about verifiability, and Peter B. Rønne thanks Steve Kremer for suggestions.

Vincenzo Iovino is supported by the Luxembourg National Research Fund (FNR grant no. 7884937). Further, this work is also supported by the INTERSequoia project from the Luxembourg National Research Fund, which is joint with the ANR project SEQUOIA ANR-14-CE28-0030-01. 


\section{References}

Adi08. Ben Adida. Helios: Web-based open-audit voting. In USENIX Security Symposium, volume 17, pages 335-348, 2008.

BBS04. Dan Boneh, Xavier Boyen, and Hovav Shacham. Short group signatures. In Matthew Franklin, editor, Advances in Cryptology - CRYPTO 2004, volume 3152 of Lecture Notes in Computer Science, pages 41-55. Springer, August 2004.

$\mathrm{BCG}^{+} 15$. David Bernhard, Véronique Cortier, David Galindo, Olivier Pereira, and Bogdan Warinschi. Sok: A comprehensive analysis of game-based ballot privacy definitions. In 2015 IEEE Symposium on Security and Privacy, pages 499-516. IEEE, 2015.

BDPA11. G. Bertoni, J. Daemen, M. Peeters, and G. Van Assche. The Keccak reference, 2011. http://keccak.noekeon.org/

BDSG $^{+}$13. Nir Bitansky, Dana Dachman-Soled, Sanjam Garg, Abhishek Jain, Yael Tauman Kalai, Adriana López-Alt, and Daniel Wichs. Why "fiatshamir for proofs" lacks a proof. In Theory of Cryptography: 10th Theory of Cryptography Conference, TCC 2013, Tokyo, Japan, March 3-6, 2013., pages 182-201. Springer, 2013.

Ben87. J. Benaloh. Verifiable secret-ballot elections. PhD thesis, Yale University, 1987.

BF03. Dan Boneh and Matthew K. Franklin. Identity based encryption from the Weil pairing. SIAM Journal on Computing, 32(3):586-615, 2003.

BFM88. Manuel Blum, Paul Feldman, and Silvio Micali. Non-interactive zeroknowledge and its applications (extended abstract). In 20th Annual ACM Symposium on Theory of Computing, pages 103-112. ACM Press, May 1988.

BFS16. Mihir Bellare, Georg Fuchsbauer, and Alessandra Scafuro. NIZKs with an untrusted CRS: Security in the face of parameter subversion. Cryptology ePrint Archive, Report 2016/372, 2016. http://eprint.iacr.org/2016/372 . To appear in ASIACRYPT 2016.

BGJS16. Saikrishna Badrinarayanan, Vipul Goyal, Aayush Jain, and Amit Sahai. Verifiable functional encryption. Cryptology ePrint Archive, Report 2016/629, 2016. http://eprint.iacr.org/2016/629. To appear in ASIACRYPT 2016.

Bit14. Nir Bitansky. Getting inside the Adversarys Head: New Directions in NonBlack-Box Knowledge Extraction. PhD thesis, Tel Aviv University, 2014.

BOV03. Boaz Barak, Shien Jin Ong, and Salil P. Vadhan. Derandomization in cryptography. In Dan Boneh, editor, Advances in Cryptology - CRYPTO 2003, volume 2729 of Lecture Notes in Computer Science, pages 299-315. Springer, August 2003.

BP15. Nir Bitansky and Omer Paneth. Zaps and non-interactive witness indistinguishability from indistinguishability obfuscation. In Theory of Cryptography Conference, pages 401-427. Springer, 2015.

BR93. Mihir Bellare and Phillip Rogaway. Random oracles are practical: A paradigm for designing efficient protocols. In V. Ashby, editor, ACM CCS 93: 1st Conference on Computer and Communications Security, pages 6273. ACM Press, November 1993. 
BSW11. Dan Boneh, Amit Sahai, and Brent Waters. Functional encryption: Definitions and challenges. In Yuval Ishai, editor, TCC 2011: 8th Theory of Cryptography Conference, volume 6597 of Lecture Notes in Computer Science, pages 253-273. Springer, March 2011.

BT94. Josh Cohen Benaloh and Dwight Tuinstra. Receipt-free secret-ballot elections (extended abstract). In 26th Annual ACM Symposium on Theory of Computing, pages 544-553. ACM Press, May 1994.

$\mathrm{CCC}^{+}$09. David Chaum, Richard Carback, Jeremy Clark, Aleksander Essex, Stefan Popoveniuc, Ronald L. Rivest, Peter Y. A. Ryan, Emily Shen, Alan T. Sherman, and Poorvi L. Vora. Scantegrity II: end-to-end verifiability by voters of optical scan elections through confirmation codes. IEEE Trans. Information Forensics and Security, 4(4):611-627, 2009.

CCFG15. Pyrros Chaidos, Véronique Cortier, Georg Fuchsbauer, and David Galindo. Beleniosrf: A non-interactive receipt-free electronic voting scheme. Cryptology ePrint Archive, Report 2015/629, 2015. http://eprint.iacr.org/2015/629. To appear in ACM CCS 2016.

CG15. Pyrros Chaidos and Jens Groth. Making sigma-protocols non-interactive without random oracles. In Public-Key Cryptography - PKC 2015 - 18th IACR International Conference on Practice and Theory in Public-Key Cryptography, Gaithersburg, MD, USA, March 30 - April 1, 2015, Proceedings, pages 650-670, 2015.

CGGI14. Véronique Cortier, David Galindo, Stéphane Glondu, and Malika Izabachène. Election verifiability for helios under weaker trust assumptions. In Miroslaw Kutylowski and Jaideep Vaidya, editors, ESORICS 2014: 19th European Symposium on Research in Computer Security, Part II, volume 8713 of Lecture Notes in Computer Science, pages 327-344. Springer, September 2014.

CGH98. Ran Canetti, Oded Goldreich, and Shai Halevi. The random oracle methodology, revisited (preliminary version). In 30th Annual ACM Symposium on Theory of Computing, pages 209-218. ACM Press, May 1998.

$\mathrm{CGK}^{+}$16. Véronique Cortier, David Galindo, Ralf Kuesters, Johannes Mueller, and Tomasz Truderung. Verifiability notions for e-voting protocols. Cryptology ePrint Archive, Report 2016/287, 2016. http://eprint.iacr.org/2016/287

CGS97. Ronald Cramer, Rosario Gennaro, and Berry Schoenmakers. A secure and optimally efficient multi-authority election scheme. In Walter Fumy, editor, Advances in Cryptology - EUROCRYPT'97, volume 1233 of Lecture Notes in Computer Science, pages 103-118. Springer, May 1997.

Cha81. David L. Chaum. Untraceable electronic mail, return addresses, and digital pseudonyms. Communications of the ACM, 24(2):84-90, 1981.

CHK04. Ran Canetti, Shai Halevi, and Jonathan Katz. Chosen-ciphertext security from identity-based encryption. In Christian Cachin and Jan Camenisch, editors, Advances in Cryptology - EUROCRYPT 2004, volume 3027 of Lecture Notes in Computer Science, pages 207-222. Springer, May 2004.

CKLM13. Melissa Chase, Markulf Kohlweiss, Anna Lysyanskaya, and Sarah Meiklejohn. Verifiable elections that scale for free. In Kaoru Kurosawa and Goichiro Hanaoka, editors, PKC 2013: 16th International Workshop on Theory and Practice in Public Key Cryptography, volume 7778 of Lecture Notes in Computer Science, pages 479-496. Springer, February / March 2013. 
CPSV16. Michele Ciampi, Giuseppe Persiano, Luisa Siniscalchi, and Ivan Visconti. A transform for NIZK almost as efficient and general as the fiat-shamir transform without programmable random oracles. In Theory of Cryptography - 13th International Conference, TCC 2016-A, Tel Aviv, Israel, January 10-13, 2016, Proceedings, Part II, pages 83-111, 2016.

CS03a. Jan Camenisch and Victor Shoup. Practical verifiable encryption and decryption of discrete logarithms. In Dan Boneh, editor, Advances in Cryptology - CRYPTO 2003, volume 2729 of Lecture Notes in Computer Science, pages 126-144. Springer, August 2003.

CS03b. Ronald Cramer and Victor Shoup. Design and analysis of practical publickey encryption schemes secure against adaptive chosen ciphertext attack. SIAM Journal on Computing, 33(1):167-226, 2003.

CS10. Veronique Cortier and Ben Smyth. Attacking and fixing helios: An analysis of ballot secrecy. Cryptology ePrint Archive, Report 2010/625, 2010. http://eprint.iacr.org/2010/625

$\mathrm{CZZ}^{+}$15. Nikos Chondros, Bingsheng Zhang, Thomas Zacharias, Panos Diamantopoulos, Stathis Maneas, Christos Patsonakis, Alex Delis, Aggelos Kiayias, and Mema Roussopoulos. A distributed, end-to-end verifiable, internet voting system. CoRR, abs/1507.06812, 2015.

$\mathrm{DDO}^{+} 01$. Alfredo De Santis, Giovanni Di Crescenzo, Rafail Ostrovsky, Giuseppe Persiano, and Amit Sahai. Robust non-interactive zero knowledge. In $A d$ vances in Cryptology - CRYPTO 2001, 21st Annual International Cryptology Conference, Santa Barbara, California, USA, August 19-23, 2001, Proceedings, pages 566-598, 2001.

DFN06. Ivan Damgård, Nelly Fazio, and Antonio Nicolosi. Non-interactive zeroknowledge from homomorphic encryption. In Shai Halevi and Tal Rabin, editors, TCC 2006: 3rd Theory of Cryptography Conference, volume 3876 of Lecture Notes in Computer Science, pages 41-59. Springer, March 2006.

DH76. Whitfield Diffie and Martin E. Hellman. New directions in cryptography. IEEE Transactions on Information Theory, 22(6):644-654, 1976.

DJ01. Ivan Damgård and Mats Jurik. A generalisation, a simplification and some applications of Paillier's probabilistic public-key system. In Kwangjo Kim, editor, PKC 2001: 4th International Workshop on Theory and Practice in Public Key Cryptography, volume 1992 of Lecture Notes in Computer Science, pages 119-136. Springer, February 2001.

DJ03. Ivan Damgård and Mads Jurik. A length-flexible threshold cryptosystem with applications. In Reihaneh Safavi-Naini and Jennifer Seberry, editors, ACISP 03: 8th Australasian Conference on Information Security and Privacy, volume 2727 of Lecture Notes in Computer Science, pages 350-364. Springer, July 2003.

DKR09. Stéphanie Delaune, Steve Kremer, and Mark Ryan. Verifying privacy-type properties of electronic voting protocols. Journal of Computer Security, 17(4):435-487, 2009.

DMP88. Alfredo De Santis, Silvio Micali, and Giuseppe Persiano. Non-interactive zero-knowledge proof systems. In Carl Pomerance, editor, Advances in Cryptology - CRYPTO'87, volume 293 of Lecture Notes in Computer Science, pages 52-72. Springer, August 1988.

DN00. Cynthia Dwork and Moni Naor. Zaps and their applications. In 41st Annual Symposium on Foundations of Computer Science, pages 283-293. IEEE Computer Society Press, November 2000. 
Fed12. Federal Agency on Technical Regulation and Metrology. Gost r 34.11-2012: Streebog hash function, 2012. https://www.streebog.net

FLS90. Uriel Feige, Dror Lapidot, and Adi Shamir. Multiple non-interactive zero knowledge proofs based on a single random string (extended abstract). In 31st Annual Symposium on Foundations of Computer Science, pages 308-317. IEEE Computer Society Press, October 1990.

FS87. Amos Fiat and Adi Shamir. How to prove yourself: Practical solutions to identification and signature problems. In Andrew M. Odlyzko, editor, Advances in Cryptology - CRYPTO'86, volume 263 of Lecture Notes in Computer Science, pages 186-194. Springer, August 1987.

$\mathrm{GGG}^{+}$14. Shafi Goldwasser, S. Dov Gordon, Vipul Goyal, Abhishek Jain, Jonathan Katz, Feng-Hao Liu, Amit Sahai, Elaine Shi, and Hong-Sheng Zhou. Multiinput functional encryption. In Phong Q. Nguyen and Elisabeth Oswald, editors, Advances in Cryptology - EUROCRYPT 2014, volume 8441 of Lecture Notes in Computer Science, pages 578-602. Springer, May 2014.

$\mathrm{GGH}^{+}$13. Sanjam Garg, Craig Gentry, Shai Halevi, Mariana Raykova, Amit Sahai, and Brent Waters. Candidate indistinguishability obfuscation and functional encryption for all circuits. In 54th Annual Symposium on Foundations of Computer Science, pages 40-49. IEEE Computer Society Press, October 2013.

GGHZ16. Sanjam Garg, Craig Gentry, Shai Halevi, and Mark Zhandry. Functional encryption without obfuscation. In Eyal Kushilevitz and Tal Malkin, editors, Theory of Cryptography: 13th International Conference, TCC 2016-A, Tel Aviv, Israel, January 10-13, 2016, Proceedings, Part II, pages 480-511. Springer, 2016.

GH07. Matthew Green and Susan Hohenberger. Blind identity-based encryption and simulatable oblivious transfer. In Kaoru Kurosawa, editor, Advances in Cryptology - ASIACRYPT 200\%, volume 4833 of Lecture Notes in Computer Science, pages 265-282. Springer, December 2007.

GIR16. Rosario Giustolisi, Vincenzo Iovino, and Peter Rønne. On the possibility of non-interactive voting in the public-key setting. In Financial Cryptography and Data Security - FC 2016 International Workshops, BITCOIN, VOTING, and WAHC, Christ Church, Barbados, February 26, 2016, Revised Selected Papers, 2016.

GK03. Shafi Goldwasser and Yael Tauman Kalai. On the (in)security of the FiatShamir paradigm. In 44th Annual Symposium on Foundations of Computer Science, pages 102-115. IEEE Computer Society Press, October 2003.

$\mathrm{GKP}^{+}$13. Shafi Goldwasser, Yael Tauman Kalai, Raluca A. Popa, Vinod Vaikuntanathan, and Nickolai Zeldovich. Reusable garbled circuits and succinct functional encryption. In Dan Boneh, Tim Roughgarden, and Joan Feigenbaum, editors, 45th Annual ACM Symposium on Theory of Computing, pages 555-564. ACM Press, June 2013.

GM84. Shafi Goldwasser and Silvio Micali. Probabilistic encryption. Journal of Computer and System Sciences, 28(2):270-299, 1984.

GO14. Jens Groth and Rafail Ostrovsky. Cryptography in the multi-string model. Journal of Cryptology, 27(3):506-543, July 2014.

Gol01. Oded Goldreich. Foundations of Cryptography: Basic Techniques, volume 1. Cambridge University Press, Cambridge, UK, 2001.

GOS06. Jens Groth, Rafail Ostrovsky, and Amit Sahai. Non-interactive zaps and new techniques for NIZK. In Cynthia Dwork, editor, Advances in Cryptol- 
ogy - CRYPTO 2006, volume 4117 of Lecture Notes in Computer Science, pages 97-111. Springer, August 2006.

Gro04. Jens Groth. Efficient maximal privacy in boardroom voting and anonymous broadcast. In International Conference on Financial Cryptography, pages 90-104. Springer, 2004.

GS08. Jens Groth and Amit Sahai. Efficient non-interactive proof systems for bilinear groups. In Nigel P. Smart, editor, Advances in Cryptology - EUROCRYPT 2008, volume 4965 of Lecture Notes in Computer Science, pages 415-432. Springer, April 2008.

GVW12. Sergey Gorbunov, Vinod Vaikuntanathan, and Hoeteck Wee. Functional encryption with bounded collusions via multi-party computation. In Reihaneh Safavi-Naini and Ran Canetti, editors, Advances in Cryptology CRYPTO 2012, volume 7417 of Lecture Notes in Computer Science, pages 162-179. Springer, August 2012.

HRZ10. Feng Hao, Peter Y. A. Ryan, and Piotr Zielinski. Anonymous voting by two-round public discussion. IET Information Security, 4(2):62-67, 2010.

JCJ10. Ari Juels, Dario Catalano, and Markus Jakobsson. Coercion-resistant electronic elections. In Towards Trustworthy Elections, pages 37-63. Springer, 2010.

Jou04. Antoine Joux. A one round protocol for tripartite Diffie-Hellman. Journal of Cryptology, 17(4):263-276, September 2004.

Kal06. Yael Tauman Kalai. Attacks on the Fiat-Shamir paradigm and program obfuscation. PhD thesis, Massachusetts Institute of Technology, 2006.

KRS10. Steve Kremer, Mark Ryan, and Ben Smyth. Election verifiability in electronic voting protocols. In European Symposium on Research in Computer Security, pages 389-404. Springer, 2010.

KSRH12. Dalia Khader, Ben Smyth, Peter Y. A. Ryan, and Feng Hao. A fair and robust voting system by broadcast. In 5th International Conference on Electronic Voting 2012, (EVOTE 2012), Co-organized by the Council of Europe, Gesellschaft für Informatik and E-Voting.CC, July 11-14, 2012, Castle Hofen, Bregenz, Austria, pages 285-299, 2012.

KY02. Aggelos Kiayias and Moti Yung. Self-tallying elections and perfect ballot secrecy. In David Naccache and Pascal Paillier, editors, PKC 2002: 5th International Workshop on Theory and Practice in Public Key Cryptography, volume 2274 of Lecture Notes in Computer Science, pages 141-158. Springer, February 2002.

KZZ15. Aggelos Kiayias, Thomas Zacharias, and Bingsheng Zhang. End-to-end verifiable elections in the standard model. In Advances in Cryptology EUROCRYPT 2015 - 34th Annual International Conference on the Theory and Applications of Cryptographic Techniques, Sofia, Bulgaria, April 2630, 2015, Proceedings, Part II, pages 468-498, 2015.

Lin15. Yehuda Lindell. An efficient transform from sigma protocols to NIZK with a CRS and non-programmable random oracle. In Theory of Cryptography - 12th Theory of Cryptography Conference, TCC 2015, Warsaw, Poland, March 23-25, 2015, Proceedings, Part I, pages 93-109, 2015.

Lip05. Helger Lipmaa. Secure electronic voting protocols. In Hossein Bidgoli, editor, Handbook of Information Security, Volume 2, Information Warfare, Social, Legal, and International Issues and Security Foundations, pages 647-657. John Wiley \& Sons, Inc., 2005. Electronic edition available at http://kodu.ut.ee/ lipmaa/papers/voting4hb.pdf 
LP09. Yehuda Lindell and Benny Pinkas. A proof of security of Yao's protocol for two-party computation. Journal of Cryptology, 22(2):161-188, April 2009.

MH96. Markus Michels and Patrick Horster. Some remarks on a receipt-free and universally verifiable mix-type voting scheme. In Kwangjo Kim and Tsutomu Matsumoto, editors, Advances in Cryptology - ASIACRYPT'96, volume 1163 of Lecture Notes in Computer Science, pages 125-132. Springer, November 1996.

MN06. Tal Moran and Moni Naor. Receipt-free universally-verifiable voting with everlasting privacy. In Cynthia Dwork, editor, Advances in Cryptology CRYPTO 2006, volume 4117 of Lecture Notes in Computer Science, pages 373-392. Springer, August 2006.

Nao03. Moni Naor. On cryptographic assumptions and challenges (invited talk). In Dan Boneh, editor, Advances in Cryptology - CRYPTO 2003, volume 2729 of Lecture Notes in Computer Science, pages 96-109. Springer, August 2003.

NY90. Moni Naor and Moti Yung. Public-key cryptosystems provably secure against chosen ciphertext attacks. In 22nd Annual ACM Symposium on Theory of Computing, pages 427-437. ACM Press, May 1990.

Riv06. Ronald L. Rivest. The threeballot voting system, 2006.

RR06. Brian Randell and Peter Y. A. Ryan. Voting technologies and trust. In IEEE Security and Privacy, pages 50-56, 2006.

RRI16. Peter Y. A. Ryan, Peter B. Rønne, and Vincenzo Iovino. Selene: Voting with transparent verifiability and coercion-mitigation. In Financial Cryptography and Data Security - FC 2016 International Workshops, BITCOIN, VOTING, and WAHC, Christ Church, Barbados, February 26, 2016, Revised Selected Papers, pages 176-192, 2016.

RS92. Charles Rackoff and Daniel R. Simon. Non-interactive zero-knowledge proof of knowledge and chosen ciphertext attack. In Joan Feigenbaum, editor, Advances in Cryptology - CRYPTO'91, volume 576 of Lecture Notes in Computer Science, pages 433-444. Springer, August 1992.

RS06. Peter Y. A. Ryan and S. A. Schneider. Prêt à voter with re-encryption mixes. Technical Report CS-TR-956, University of Newcastle, 2006.

RT09. Peter Y. A. Ryan and Vanessa Teague. Pretty good democracy. In IN: WORKSHOP ON SECURITY PROTOCOLS, 2009.

SK95. Kazue Sako and Joe Kilian. Receipt-free mix-type voting scheme - a practical solution to the implementation of a voting booth. In Louis C. Guillou and Jean-Jacques Quisquater, editors, Advances in Cryptology - EUROCRYPT'95, volume 921 of Lecture Notes in Computer Science, pages 393403. Springer, May 1995.

SS10. Amit Sahai and Hakan Seyalioglu. Worry-free encryption: functional encryption with public keys. In Ehab Al-Shaer, Angelos D. Keromytis, and Vitaly Shmatikov, editors, ACM CCS 10: 17th Conference on Computer and Communications Security, pages 463-472. ACM Press, October 2010.

Yao86. Andrew Chi-Chih Yao. How to generate and exchange secrets (extended abstract). In 27th Annual Symposium on Foundations of Computer Science, pages 162-167. IEEE Computer Society Press, October 1986. 